\title{
INDEX ALPHABÉTIQUE DES ARTICLES (CONCEPTS): DAG 22
}

accorder une sauveté à (une

ville, un lieu) 2676

accueil v. réception

action de conduire 2674

agriculteur v. paysan

aide, assistance, secours 2654

aide, auxiliaire, appui (personne) 2656

aide militaire 2657

aider qn 2655

aiguillon (du bouvier) 2727

aiguisage (droit perçu pour l'des outils agricoles) 2733

aiguiser 2732

aiguiser (celui qui fait - ses outils agricoles) 2734

amender le sol (en parl. du bétail) 2696

appel au secours 2658

appentis v. hangar

appui v. aide 2656

assistance v. aide 2654

attenant, limitrophe 2715

attenant (terrain - à une propriété) 2716

auxiliaire v. aide 2656

avoine (celui qui donne de l'aux chevaux) 2691

barrière 2723

bête de labour 2755

blé (champ de -) 2765

borne 2724

botte de foin 2745

bouvier 2726

bouvier (aiguillon du -) 2727

celui qui donne de l'avoine aux chevaux 2691

celui qui fait aiguiser ses outils agricoles, le soc de sa charrue 2734

champ 2746

champ (convertir en -) 2748

champ de blé 2765

champ de fourrage 2747 charrue 2756

charrue (pièces de la-) v. ferrures

charrue (soc de -) 2734, 2759

conduire, guider 2673

conduire (action de -) 2674

convertir en champ 2748

cour 2704

coutre 2758

creuser une rigole 2720

cultive (qui -, laboure la terre) 2678

cultiver 2680

défendre qn, qch v. protéger 2659

défense, protection 2660

défenseur, protecteur, soutien 2661

défriché(e) (terre/terrain -) 2711

défricher 2712

délimiter une terre, un terrain 2717

dépérir (d'une bête) 2702

dévorer 2692

donner sauf-conduit 2672

donner une quatrième façon à la terre 2752

droit de faucher 2736

droit perçu pour l'aiguisage des outils agricoles 2733

élever (un animal) v. nourrir en sauvegarde et en sécurité 2669

endroit où est entreposée la paille v. pailler

ensemencer v. semer

entourer d'un fossé 2722

épuisé (d'un bovin) v. sub maigre

étable 2687

façon donnée au sol, à la terre 2751 façon (donner une quatrième à la terre) 2752

faire connaître (qn à qn) v. présenter (qn à qn)

faner, couper et récolter les foins 2743

faucher 2735

faucher (droit de -) 2736

faux 2731

fenaison 2742

ferme, métairie 2681

fermier, métayer 2679

ferrures, pièces de la charrue 2757

fixation des limites de terre 2713

foin 2740

foin (botte de -) 2745

foin (prairie à -) 2741

foin (meule/tas de -) 2744

foins (faner, couper et récolter les -) 2743

fosse à fumier 2695

fossé (entourer d'un -) 2722

fossé qui sert de limite aux terrains 2721

fourche 2737

fourrage (champ de -) 2747

fumer la terre 2697

fumier animal 2694

fumier (fosse à -) 2695

fumure 2698

garant v. protecteur 2665

garantir qch v. protéger 2664

garde, protection 2666

garde des moissons 2767

garder v. protéger 2662

gardien des moissons, des récoltes 2768

grange 2684

guider v. conduire

hangar, appentis 2685

herser 2763

hiverner (du bétail) 2693 
joug (soumis au -) 2725

labour 2754

labour (bête de -) 2755

laboure (qui cultive, - la terre) 2678

labourée (terre -) 2753

labourer (la terre, le champ, la vigne) v. travailler

laisser se reposer un an (de la terre) 2764

lieu d'asile v. sauveté

lieu solitaire 2651

limite (d'un terrain, d'un champ) 2714

limite (fossé qui sert de - aux terrains) 2721

limiter un terrain par une ou plusieurs rigoles 2718

limites (fixation des - de terre) 2713

limitrophe v. attenant

limitrophe (propriété -) 2716

maigre, épuisé (d'un bovin) 2699

maison d'habitation d'une ferme 2683

maître d'un casal v. tenancier

malade (animal) 2700

malade (tomber -, animal) 2701

métairie v. ferme

métayer v. fermier

meule de foin, tas de foin 2744

moisson 2766

moissonner 2769

moissonneur 2770

moissons (garde des -) 2767

moissons (gardien des -) 2768

nourrir, élever (un animal) 2689

nourriture du bétail 2690

pailler, endroit où est entreposée la paille 2686 parcelle de terre $\mathrm{v}$. pièce de terre

paysan, agriculteur 2677

pièce, parcelle de terre 2706

pièces de la charrue $v$. ferrures prairie 2728

prairie (transformer en -) 2730

prairie à foin 2741

pré 2729

premiers produits de la terre, première récolte 2771

préparer la terre 2749

présenter, faire connaître (qn à qn) 2653

préservation v. protection 2663

préserver v. protéger 2662

protecteur, garant 2665

protecteur v. défenseur 2661

protection, préservation 2663

protection v. garde

protéger, défendre qn, qch 2659

protéger, garantir qch 2664

protéger, garder, préserver 2662

qui cultive, laboure la terre 2678

qui tète (jeune animal) 2688

râteau 2738

râteler, ratisser 2739

ratisser v. râteler

réception, accueil 2649

récolte (première -) 2771

récoltes (gardien des -) 2768

reposer (laisser se - un an, de

la terre) 2764

rigole 2719

rigole (creuser une -) 2720

rigoles (limiter un terrain par

une ou plusieurs -) 2718

sain (animal) 2703

salut 2652 sauf et sûr (d'un lieu) 2670

sauf, sauvegardé 2668

sauf-conduit 2671

sauf-conduit (donner -) 2672

sauvegarde 2667

sauvegarde (en - et en sécurité)

2669

sauvegardé v. sauf

sauveté, lieu d'asile 2675

sauveté (accorder une - à une

ville, un lieu) 2676

secours v. aide 2654

secours (appel au-) 2658

sécurité (en sauvegarde et en -)

2669

semence 2762

semer, ensemencer 2761

sillon 2760

soc de charrue 2759

soc (le - de sa charrue) 2734

solitaire 2650

solitaire (lieu -) 2651

soumis au joug 2725

soutien v. défenseur 2661

sûr (sauf et -, d'un lieu) 2670

tas de foin v. meule de foin

tenancier, maître d'un casal

2682

terrain, terre 2705

terrain attenant à une propriété, propriété limitrophe 2716

terrain broussailleux 2707

terrain sec 2708

terre v. terrain

terre cultivée 2710

terre en friche v. terre inculte

terre inculte, en friche 2709

terre labourée 2753

terre/terrain défriché(e) 2711

tète (qui -, jeune animal) 2688

tomber malade (animal) 2701

transformer en prairie 2730

travailler, labourer (la terre, le champ, la vigne) 2750 
Mill [v. l'attestation ci-dessus]; DOM 141b sub aculhir 1c «recevoir favorablement, souhaiter la bienvenue à, donner l'hospitalité à».

2. arcoalher v.tr. 1294 ArbitrTarbesR 5[2; DocBigJ 164 1. arcoeilher].

FEW COLLIGERE 2,901a: béarn. arcoelhe «accueillir, recevoir» [= LR: aussi abéarn. arcoelher [s.d.] ArchBPyr].

3. emparar v.tr. 1294 ArbitrTarbesR 6[5;11].

FEW PARARE 7,631b: apr. amparar «acueillir» Lv.

4. receber v.tr. 1294 ArbitrTarbesR 6[10], recebre [13 ${ }^{\mathrm{e}}$ s.; cop. ca. 1400] ACoutBordB 180[238]; arceber 1294 ArbitrTarbesR 7[11].

FEW RECIPERE 10,145a: apr. recebre v.a. «accueillir (qn) de telle ou telle manière»; LR sub recebe: abéarn. arceber «recevoir (qn)» [av. 1400; cop. $15^{\mathrm{e}}$ s.] ForAspe, ForsB[OG] 546[12: var.].

5. «recevoir (qn) en sa maison, accueillir (qn) chez soi» arecebre en son ostau synt.verb. [ca. 1290] HommVayresD, ArchGir 8,43.

À rattacher à FEW RECIPERE 10,145a et HOSPITALIS 4,493a/b.

6. «accueillir chez soi, donner asile chez soi (à un malfaiteur)» a) receber v.tr. 1247 [cop. ca. 1460] ForAspe, ForsB[OG] 532[2].

ø FEW RECIPERE 10,145a.

b) receber en son hostau synt.verb. [ca. 1220; cop. ca. 1460] ForGén, ForsB[OG] 246[185]; 1267 [cop. ca. 1460] ForOss, ForsB[OG] 522[17].

À rattacher à FEW RECIPERE 10,145a et HOSPITALIS 4,493a/b.

c) receber en sa mayson synt.verb. [1220; cop. ca. 1460] ForMorl, ForsB[OG] 332[38].

À rattacher à FEW RECIPERE 10,145a et MANSIO $6^{1}, 235$.

1 aculer

1277 [cop.] Loubens: He auem los plus afranquid e asot . . . a les sorors e als frais ... que tot hom qui sie de noste poder se pusque far de le auant dite maison, se hidz 1-i uolint aculer, so que casau no-n descr[o]bie.

\section{2 arcoalher}

1294 Tarbes: fo ordenad . . que de paroent a de batilhe pague hom .XV. s(oos) de morl(aas) ... e e si pagar no pode que fos farid de totes tres las beziaus trou que pagas, e si arres de las beziaus l-arcoalhe en som poder aqued que pagas la lei.

3 emparar

1294 Tarbes: si nuil bezii D-ivoz [1.: d'Ivoz] aucizo [texte illisisble] que las beziaus de Tarbe ni de Baigneres no 1-emparassen neit ni die. 


\section{4 receber}

1294 Tarbes: fo ordenad . . que la beziau de Tarbe per cau que negoci fos fore de Tarbe o augun lor bezii podos tornar a Baigneres e a Yvos, ei fossen recebudz.

5 arecebre en son ostau

[ca. 1290] Vayres [Gir.]: li home d'Ardilars ... sobre lor deuen lo [scil. lo senhor de Bayras] arecebre en lur ostau ses gran grenche.

6a receber

1247 [cop. ca. 1460] Aspe: ad aqueg qui ad atau [scil. un tel malfaiteur] deffenere, thiere o recebere, que pusque far aquero medix.

$6 \mathrm{~b}$ receber en son hostau

[ca. 1220; cop. ca. 1460] Béarn: si per aventure nulhs hom d'esta biele recebe en son hostau lo homicidi, per cascun die que l'i thiere pague au senhor .LXVI. ss (v. aussi sub aide, assistance, secours 2654,3).

6c receber en sa mayson

[1220; cop. ca. 1460] Morlaàs: si per abenture nulhs hom d'esta biele recebe l'omicidi en sa mayson, per cascun die qui l'i thiere pague au senhor .LXVI. ss.

\section{9 réception, accueil:}

1. receptio f. [1288; cop. ca. 1300] CoutMontsaunèsM 53a[51].

ø FEW RECIPERE 10,147b; Rn 2,280a: receptios pl. «réceptions (en parl. des religieuses)»1319 Doat [agasc. ou alang.].

1 receptio

[1288; cop. ca. 1300] Montsaunès: Receptio del comte de Cumenge.

\section{0 solitaire:}

1. «personne seule (ici, personne qui marche toute seule en route)» soletari (que ani per le carrere) m. [1273] ACoutBay, Bal 2,648[8] = LR sub boilhon.

$ø$ FEW SOLITARIUS 12,61a.

\section{1 lieu solitaire:}

1. loc soletari loc.m. [1273] ACoutBay, Bal 2,652[19].

À rattacher à FEW LOCUS 5,392a et SOLITARIUS 12,60b; LR sub soulitari: abéarn. loc soletari en que gentz no aguos «un lieu solitaire, où il n'y eût personne» $15^{\mathrm{e}} \mathrm{s}$. BaronBéarn. 


\section{1 loc soletari}

[1273] Bayonne: Si nuytaumentz o midiaumentz en loc soletari en que gentz no aguos augun ha aucit, ...

bb) L'étiquette, la convenance

\section{2 salut:}

1. «formule exclamative de souhait, de civilité, employée spécialet. dans le préambule des lois et ordonnances, dans les lettres patentes des rois, etc.» salutz pl. 1199 [cop. ca. 1400] ACoutBordB 445; GrosclaudeGasc 35; [1262; cop. ca. 1400] Pad, ACoutBordB 187.

$ø$ FEW SALUS 11,126a: fr. salut dp. 1316; LR sub salut (anciennement du g[enre] fém.): abéarn. salud «salut» ca. 1330 [cop. ca. 1410] HistSainte, ib. sub bayle: salutz (Gaston au baile de Pau) [ca. 1400] ForsB.

$\diamond$ Rem. Le genre ne ressort pas clairement des citations.

\section{1 salutz $\mathrm{pl}$.}

1199 [cop. ca. 1400] Bordeaux: Johan, per la gracia de Diu, Rey d'Anglaterra . . . aus senescaus, vescomtes, perbost e a totz bailieus fideus sotz, salutz.

\section{3 présenter, faire connaître (qn à qn):}

1. presentar v.tr. 1259 Mill, ArchGir 45,3[1,8]; [13 s.; cop. ca. 1460] ForGén, ForsB[OG] 152[18].

ø FEW PRAESENTARE 9,308b.

2. mostrar v.tr. 1171 [cop. 1251] Bagnères-de-Bigorre, RecGascL 21[17].

$\varnothing$ FEW MONSTRARE $6^{3}, 95$ a.

3. demustrar v.tr. 1294 [cop. 1343] CoutLect, ArchLectD 43[50].

ø FEW DEMONSTRARE 3,38a.

\section{1 presentar}

[13 ${ }^{\mathrm{e}}$ s.; cop. ca. 1460] Béarn: que totz los cavers de Bearn deben lors filhs presentar au senhor, exceptat aqueg qui volera que sie son successor a ssa terre.

2 mostrar

1171 [cop. 1252] Bagnères-de-Bigorre: E que-ls dam e-ls autrejam als borgs de Banheres que si nulh home i benia per bezin ester e sera mostrad em bezial per bezin.

\section{3 demustrar}

1294 [cop. 1343] Lectoure: la universitat e-1 cominal de Laytora pot e deu elegir 
cosselhs o-l meys coselh autra coselh successioument ab voluntat del dit cominal ... e en apres, ... deuo esser demustratz als predits senhors o a lors bailes.

cc) L'entr'aide

\section{4 aide, assistance, secours:}

1. ajude f. [1220; cop. ca. 1475] v. benir en soccors et ajude sub aider qn 2655,7, ajuda 1238 [vid. 1278; cop. 14 s. s.] PrivEDM[D], ArchGir 3,117; [ca. 1250] CartBigRC [60,23: ms. B, var. de aida, ci-dessous 2].

FEW ADJUTARE 24,162a/b: apr. ajuda f. «aide, secours» fin $12^{\mathrm{e}} \mathrm{s}$., Rn; etc., Gers ajido «secours, assistance», gasc. ayude 1776, LaF, béarn. id., ajude; LR: abéarn. ajude, aiude «aide, secours» [s.d.] ArchBPyr, ib. sub regnat: ajude ca. 1330 [cop. ca. 1410] HistSainte, ib. sub counfort: $15^{\mathrm{e}} \mathrm{s}$. BaronBéarn; DOM 492a sub ajuda f).

2. aida f. [ca. 1250; cop. ca. 1390] CartBigRC 79[60,25-26: ms. Pa; interprété comme verbe dans le gloss et dans la trad. de l'éd.: aidar a (qn)].

FEW ADJUTARE 24,162a/b: apr. aida f. «aide, secours» 1476 Pans, béarn. ayde «secours, assistance» [= LR]; DOM 360a sub a).

3. adjutori m. 1125 [cop. après 1425] CartBigRC 66[44,48: ms. $\mathrm{Pb}$ ]; adiutori [ca. 1175; cop. ca. 1390] CartBigRC 59[41,9]; 1260 CoutBagn, MusArch 168; RecGascL 33 [adiutori]; id. 1267 [cop. ca. 1460] ForOss, ForsB[OG] 522[17]; 1278 [cop. ca. 1460] ForOlDC 99a[1];117a[16] = LR; Rn 3,610a: 1080 [date se référant au document original perdu, fort probablement écrit en latin], ajutori 1277 [cop. $15^{\mathrm{e}} \mathrm{s}$.] CartOssauT 53; 1278 [cop. 1551] CartOloron, ForOlDC 98a[1];116a[16].

FEW ADJUTORIUM 24,164b: apr. a(d)jutori m. «aide, secours» 1080 [datation d'après Rn, v. ci-dessus]-1317, Rn; etc., Gers, béarn. adjutori; DOM 504/505 sub ajutori.

4. soccors m. [1220; cop. ca. 1460] ForMorl v. benir en soccors sub aider qn 2655, 7; socirs [forme corrompue ?] 1277 [cop. 15 ${ }^{\text {es. }}$ CartOssauT 53.

FEW SUCCURRERE 12,383a: béarn. secous m. «aide, assistance dans le danger, dans le besoin»; LR sub secous: abéarn. socos «secours» ca. 1330 [cop. ca. 1410] HistSainte, ib. sub fabou: [s.d.] ArchBPyr.

5. cosselh m. 1171 [cop. 1251] Bagnères-de-Bigorre, RecGascL $21=$ Lv 1,334b sub 5.

FEW CONSILIUM 2,1071b: apr. conselh «secours».

6. «force, secours, main-forte»

force f. 1125 [cop. après 1425] CartBigRC 66[44,39: ms. Pb]; [ca. 1175; cop. ca. 1390] CartBigRC 59[41,9: ms. Pa] = Lv 3,561b sub 3 «aide, secours». 
FEW FORTIA 3,726a: apr. forsa «aide, secours».

$\diamond$ Rem. En combinaison avec adiutori «aide» il faut comprendre «force; secours». Les sémantismes de force et adiutori se recouvrent largement (CartBigRCGloss sub force).

\section{1 ajuda}

1238 [vid. 1278; cop. 14 ${ }^{\mathrm{e}}$ s.] Entre-deux-Mers: si lo perbost del seynhor Rey volesse pignorar aucun rebelle ..., podesse trametre a las quaus parropias que volgues per aucuns homes expeditz a ajuda. - [ca. 1250] Lourdes: Lo casal de Ferrad ceissament sens pa fer e aiuda a ' $\mathrm{n}$ Bonel de Clauaria a las forcas dels penudz.

\section{2 aida}

[ca. 1250; cop. ca. 1390] Lourdes: lo casal de Ferrat . . . aida a 'n Boneu de Clauaria a las forcas dels penutz.

\section{3 adjutori}

1267 [cop. ca. 1460] Ossau: [en parl. d'un malfaiteur] si per abenture augun deus Ossalees aquest atau recebe en son hostau et adiutori lo dave ... (v. aussi ci-dessous 6; ajutori v. ci-dessous 4).

4 socirs [?]

1277 [cop. $15^{\mathrm{e}}$ s.] Ossau: si auguns de la val d'Ossau e de la bal de Camfranc auran prees injurie et dampnages de tau se bol autes gentz e la une part de aqueres requerira en socirs [sic] et ajutori.

6 force

1125 [cop. après 1425] Bigorre: Sans Gassia . . . lo [= al comte de Bigorre] pleui . . . que force e adjutori e amig e fidel lo fos de totz homes del mon.

\section{5 aider qn:}

1. «apporter son aide»

ajudar v.intr.: aiudar 1171 [cop. 1251] Bagnères-de-Bigorre, RecGascL 24; 1275 Bordeaux [concern. Salles et Mios], ArchGir 25,490.

ø FEW ADJUTARE 24,161a.

2. «porter aide, secours, soutien à (qn)»

a) ajudar (qn) v.tr.: aiudar [ca. 1114; cop. ca. 1390] CartBigRC 21[7,18: ms. Pa]; id. [ca. 1175; cop. ca. 1390] CartBigRC 59[41,10: ms. Pa]; [ca. 1190] ChMalf, Bal 1,423; id. 1203 [cop. 1345] CoutSGaudensM 58[77]; id. 1264 Bordeaux [concern. Bayonne], Bal 2,593[3]; id. 1294 [cop. 1342] CoutLect, ArchLectD 35.

FEW ADJUTARE 24,161a/162a: apr. ajuar «aider» $15^{\mathrm{e}}$ s., MeyerDoc; Fazy, Gers ahitá, béarn. ajudá; LR sub infourma: abéarn. ajudar (qn) «aider (qn)» $15^{\mathrm{e}} \mathrm{s}$. BaronBéarn; DOM 497b sub ajudar 3a). 
b) aidar (qn) v.tr. [ca. 1175; cop. ca. 1250] CartBigRC [41,10: ms. B, var. de aiudar (qn), ci-dessus 2a)], eydar (qn) 1275 [cop. 1358] Bordeaux, ArchGir 31, 245 .

FEW ADJUTARE 24,162a: béarn. aydá «aider»; Rn 3,610b: alang. aizar (qn) $14^{\mathrm{e}}$ s. LSydrac; LR sub ayda: abéarn. aydar «aider» [s.d.] ArchBPyr.

c) ajudar a (qn) v.tr.: aiudar [ca. 1140; cop. ca. 1390] CartBigRC 67[47,7: ms. Pa]; [12 s.; cop. ca. 1460] ForsB[OG] 144[6];162[36]; 1238 [vid. 1278; cop.14 s.] PrivEDM[D], ArchGir 3,121; 1241 DocSEm, ArchGir 28,496; [1258; cop. $16^{\mathrm{e}} \mathrm{s}$.] CoutLaRéoleG, ArchGir 2,266[48]; 1267 [cop. ca. 1460] ForOss, ForsB[OG] $518[8]$.

FEW ADJUTARE 24,161a: apr. ajudar v.a.n. «aider»; Rn 3,609a: apr. ajudar a (qn) «aider à» ca. 1227 Jaufre; LR sub teme: abéarn. ajudar a «aider qn» ca. 1330 [cop. ca. 1410] HistSainte; DOM 495b sub ajudar 2a).

d) valer (qn) v.tr. 1270 [cop. 1380] ChartSSeverPC 478[trad. «être utile à (qn)»]. $\varnothing$ FEW VALERE 14,131a; cf. LR sub bale: abéarn. baler (qn) «aider, protéger (qn)» ca. 1330 [cop. ca. 1410] HistSainte.

e) baler a (qn) de (qch) v.tr. 1241 DocSEm, ArchGir 28,496.

FEW VALERE 14,131a: abéarn. baler, bale (à qn) «être utile à, aider».

f) seguir a (qn) v.tr. 1270 [cop. 1380] ChartSSeverPC 478[trad. «suivre (qn)»]; [1273] ACoutBay, Bal 2,615[54].

ø FEW SEQUI 11,488b/489a; Lv 7,518a sub 2: apr. segre «helfen, beistehen ?» 1272 CoutSBonnet.

3. «porter aide à (qn) contre (qn)»

a) ajudar (qn) de (qn) v.tr.: aiudar [12 $\mathrm{e}$ s.; cop. ca. 1460] ForGén, ForsB[OG] 162[35]; [13 ${ }^{\mathrm{e}} \mathrm{s}$; cop.] CoutMeilhanB, ArchGir 25,143[29]; ajudar (qn) de (qch): aiudar 1253 Sauveterre-de-Béarn, RecGascL 52[28].

ø FEW ADJUTARE 24,161a; DOM 496a sub ajudar 2f) et 499a sub m) «protéger qn contre, préserver qn de».

b) valer (qn) de (qn) v.tr. [13 ${ }^{\mathrm{e}}$ s.; cop.] CoutMeilhanG, ArchGir 25,143[29: l'éd. trad. «prendre la défense de qn»].

ø FEW VALERE 14,131a; cf. Lv 8,576a sub 11: apr. valer de «helfen in betreff . ..»ca. 1125 RaimVidal.

c) ajudar (qn) contra (qn) v.tr. 1238 [vid. 1278; cop. 14e s.] PrivEDM[D], ArchGir 3,120.

$\varnothing$ FEW ADJUTARE 24,161a; DOM 495a sub 1g): aoccit. ajudar (qn) contra «protéger qn contre». 
4. «aider (qn) dans (qch)»

ajudar (qn) en (qch) v.tr. [1220; cop. ca. 1460] ForMorl, ForsB[OG] 330[36].

ø FEW ADJUTARE 24,161a; Rn 3,609a: alang. ajudar (qn) en «aider (qn) en» 1034; DOM 498b sub 3i) «aider (qn) dans [une affaire, une situation]».

5. «aider, secourir», ici «prendre part à (qch) à titre d'auxiliaire»

baler de (qch) v.tr. [ca. 1238; cop. ca 1390] CartBigRC 106[71,122].

ø FEW VALERE 14,131a; Lv 8,576a sub 11 valer de «helfen in betreff . . .»: apr. en valer ca. 1190 BertBorn.

6. «porter secours à (qn)»

socorrer (a) (qn) v.tr. 1264 Bordeaux [concern. Bayonne], Bal 2,593[3].

FEW SUCURRERE 12,382b/383a: apr. socorrir "secourir», secorrir $13^{\mathrm{e}} \mathrm{s}$., béarn. secouri; cf. Lv 7,737b: agasc. secorrer (qn) de (qch) «porter secours à (qn) au moyen de (qch)» Bordeaux 1407; LR sub secorre: abéarn. secorrer $a$ (qn) «porter secours à (qn)» ca. 1330 [cop. ca. 1410] HistSainte.

7. «venir à la rescousse, au secours (de qn)»

benir en soccors loc.verb. [1220; cop. ca. 1460] ForMorl, ForsB[OG] 312[3]; benir en soccors et ajude [1220; cop. ca. 1475] ForMorl, ForsB[OG] 312[3: var.].

À rattacher à FEW VENIRE 14,240a, SUCURRERE 12,383b et ADJUTARE 24,162a/b; cf. Rn 2,494a: apr. far ajuda e secors "faire aide et secours» ca. 1175 ArnMar; DOM 492a sub ajuda h).

8. «aider, assister (qn) (?)»

tier/tir (qn) v.tr. 1291 [cop. 1336?] EtBay 91[93].

ø FEW TENERE $13^{1}$,209ss.; Lv 8, 151 b sub 17 «unterstützen, beistehen (?)»: arouerg. tener (qn) ca. 1480 MystRouergue.

$\diamond$ Rem. Le sens plus restreint «accueillir, cacher chez soi» (FEW 13 ${ }^{1}, 216 \mathrm{~b}$ sub g) n'est pas à exclure.

9. «aider (qn) à faire (qch)»

a) ajudar $\boldsymbol{a}$ (qn) $\boldsymbol{a}$ (faire qch) v.tr. [ca. 1175; cop. après 1425] CartBigRC [36, 3: var. $\mathrm{Pb}$; ms. Pa et ms. B: aiuda], ajudar [a] (qn) a [+ inf.]: aiudar 1270 [cop. 1380] ChartSSeverPC 478.

ø FEW ADJUTARE 24,161a; DOM 496a sub ajudar 2e).

b) aidar a (faire qch) 1179 Comminges, Brunel 161[172,32]; RecGascL 6[4].

ø FEW ADJUTARE 24,161a; DOM 361a sub aidar 2c) «contribuer à, concourir à» 1179. 
10. «aider, assister (qn) dans (qch), suivre, soutenir (qn) pour (faire qch)» seguir (qn) v.tr. [+ inf.] 1247 [cop. ca. 1460] ForAspe, ForsB[OG] 530[1]; seguir (qn) $\boldsymbol{a}$ [+ inf.] 1267 [cop. ca. 1460] ForOss, ForsB[OG] 522[15].

ø FEW SEQUI 11,488b/489a; cf. Lv 7,518a sub 2: apr. segre «helfen, beistehen?» 1272 CoutSBonnet.

11. «S'aider réciproquement, se devoir aide»

a) s'ajudar v.pron.: s-aiudar 1294 ArbitrTarbesR 6[9]; [13 ${ }^{\mathrm{e}} \mathrm{s}$; cop.] CoutMeilhanB, ArchGir 25,143[29;30].

ø FEW ADJUTARE 24,161a; LR sub ajuda: béarn. ajuda-s «s'aider».

b) se baler v.pron. 1294 ArbitrTarbesR 6[9], se valer [13 ${ }^{\mathrm{e}}$ s.; cop.] CoutMeilhanB, ArchGir 25,143[29;30].

$ø$ FEW VALERE 14,131a.

\section{1 ajudar}

1171 [cop. 1251] Bagnères-de-Bigorre: si nulh hom feriue autre en la gleize . . . qu'i deuem auer.V. sols de ley: e si autre hom qi defores fos i entraue per aiudar e i ferive maliciosamentz . . . que en deuem auer .LX. e.V. sols de lei.

2a ajudar (qn)

[ca. 1114; cop. ca. 1390] Barèges: la comtessa ... torna als homes de Baredge e prega-ls gran mercet que la coselhasan e l'aiudassan (v. aussi ci-dessous 2e; sub aide militaire 2657,1).

$2 \mathrm{~b}$ aidar (qn)

[ca. 1175; cop. ca. 1250] Lourdes: ... pliuirin lo qe amigs e fidels lo fossan per dreita fe e esteus enganc e force e adiutori contra totz los homes del mon, esters que del comte d'Estarag no l'aidassen.

2c ajudar a (qn)

[ca. 1114; cop. ca. 1390] Barèges: que ... l'aiudassan (v. ci-dessus 2a).

$2 \mathrm{~d}$ valer (qn)

1270 [cop. 1380] SSever: ... ans [li home de la vila de Sent-Sever] los i deven ajudar et valer a bona fe.

2e baler a (qn) de (qch)

1241 SÉmilion: . . . qu'en ajudes e- $n$ baugos à l'autra partida.

$2 \mathrm{f}$ seguir $a$ (qn)

1270 [cop. 1380] SSever: Et es assaber que lodeits bayles ... no deu embaigar que li home de la vila de Sent-Sever ... no ajuden et no seguian a l'abat et au combent deudeit moster.

3a ajudar (qn) de (qn)

[12 ${ }^{\mathrm{e}}$ s.; cop. ca. 1460] Béarn: no es a lexar que au senhor totz sons homis, cavers et autres, lo deven aiudar de sons adversaris. 
$3 \mathrm{~b}$ valer (qn) de (qn)

[13 ${ }^{\mathrm{e}}$ s.; cop.] Meilhan-sur-Garonne: juret que si nulhs hom faze mal ni tort à cavoir o à borgues de Milhan ... que tut l'en ajudessen e l'en valgossen e l'en deffendessen leyalment.

$3 \mathrm{c}$ ajudar (qn) contra (qn)

1238 [vid. 1278; cop. $14^{\mathrm{e}}$ s.] Entre-deux-Mers: et per jurament se colliga a ajudar una part contra l'autre a ffar guerra.

4 ajudar (qn) en (qch)

[1220; cop. ca. 1460] Morlaàs: [en parl. du seigneur] que-us [les hommes] ajudi en totes causes.

\section{5 baler de (qch)}

[ca. 1238; cop. ca. 1390] Bigorre: La biela de Lorda deu carnalar ab homes de Polzag en baler.

6 socorrer (a) (qn)

1264 Bordeaux [concern. Bayonne]: socorrossen (v. sub aide militaire 2657,1).

7 benir en soccors

[1220; cop. ca. 1460] Morlaàs: Si augun homi fer autre en glisie ... si augun biene de deffore en soccors et ferive yradementz ad autre, dara au senhor .LXVI. ss.

8 tier/tir (qn)

1291 [cop. 1336?] Bayonne: que nulhs hom o femne estrainh ni priuat no empari ni tinque murtrer ni banitz.

9a ajudar $[a]$ (qn) $a$ (faire qch)

1270 [cop. 1380] SSever: et l'abat e'u combent Sent-Sever e-us aiude a defendre.

$9 \mathrm{~b}$ aidar $\mathrm{a}$ (faire $\mathrm{qch}$ )

1179 Comminges: . . . qui tot ag parlá e ag aidá a fér.

10 seguir (qn)

1247 [cop. ca. 1460] Aspe: Et si tote la terre no-u seguive far totes lasdites penheres, que eg pusque penherar tote la terre d'Aspe.

11a s'ajudar

1294 Tarbes: que de toz los homes deu comdad de Begorre s-aiuden e-s bailhen a lors poders (v. aussi ci-dessous 11b).

$11 \mathrm{~b}$ se valer

[13 ${ }^{\mathrm{e}}$ s.; cop.] Meilhan-sur-Garonne: e-l borgues $s$ 'en deven ajudar e valer (se baler v. ci-dessus 11a). 


\section{6 aide, auxiliaire, appui (personne):}

1. «personne qui apporte à une autre son appui»

ajudedor m. 1270 Casteljaloux, RecGascL $96=\mathrm{Lv}$ 8,569a/b sub valedor 5.

FEW ADJUTARE 24,163a: apr. ajudador (ajudaire nom.) «auxiliaire, aide» 1195-ca. 1425, Brunel; Rn [«auxiliaire» 1248, Doat (alang. ou agasc.?)]; Pans, béarn. ajuadoú; LR sub ajudadou: abéarn. ajudador, aiuededor «aide» [s.d.] ArchBPyr.

2. baledor m. 1243 [cop. ca. 1480] CartDax: LNoirDaxA, ArchGir 37,213, gloss «allié, partisan»= Lv 8,569b «Helfer», ualedor 1270 Casteljaloux, RecGascL 96 $=\mathrm{Lv}$.

FEW VALERE 14,133a: abéarn. baledor m. «auxiliaire, aide» [=LR: «auxiliaire, allié» ca. 1377 RôlesGPhébus]; Rn 3,610a: alang. ajudador ni valedor «aide ou soutien» ca. 1420 CatApostRoma.

$\diamond$ Rem. Cf. LR sub balence: abéarn. esser de la balence de (qn) «être du corps d'auxiliaires de (qn)» [s.d.] ArchBPyr désignant les gens attachés comme auxiliaires au parti de qn.

3. «personne qui favorise, protège (qn), fauteur»

fautor m. 1243 [cop. ca. 1480] CartDax: LNoirDaxA, ArchGir 37,213, gloss «fauteur, partisan» $=\operatorname{Lv} 8,569 \mathrm{~b}$ sub valedor 5 .

ø FEW FAUTOR 3,439a; Rn 3,287b: alang. fautor «fauteur» ca 1420 CatApostRoma.

\section{7 aide militaire:}

1. «aide, secours (en hommes et en vaisseaux)» secors (de gens et de naus) m. 1264 Bordeaux [concern. Bayonne], Bal 2,593[3].

FEW SUCURRERE 12,383a: apr. socors m. «aide, assistance dans le danger, dans le besoin», secors $13^{\mathrm{e}}$ s., socor, secor $13^{\mathrm{e}}-14^{\mathrm{e}}$ s., béarn. secous [= LR: 1583 Salette, aussi abéarn. socos «secours» ca. 1330 [cop. ca. 1410] HistSainte, ib sub fabou: socos [s.d.] ArchBPyr].

1 secors

1264 Bordeaux [concern. Bayonne]: Sapiadz que cum nos de les partz de nostre seignor Haudoard aossem pregat lo maire e-us iuradz . . . que 1-aiudassen et socorrossen $a b$ gens et ab naus, en le so grant coite $\mathrm{D}$-anglaterre ... que eds de lor gracie nos aien feite bone esposte e ne meton secors de gens et de naus.

\section{8 appel au secours (t.jurid.):}

1. «à l'aide, au secours (cri de détresse): appel à l'aide de ses concitoyens 1678 
émanant d'une personne troublée dans sa sécurité personnelle ou dans la possession de ses biens»

(crit de, cridar) biaffora: audir lo crit de Biaffora 1238 [cop. 14e s.] PrivEDM[D], ArchGir 3,120;123 [cridar o clamar: Biaffora], byaffora: cridar: Byaffora $\left[13^{\mathrm{e}} \mathrm{s}\right.$;; cop. ca. 1400] ACoutBordB 107[134], biafora: cridar: Biafora $\left[13^{\mathrm{e}} \mathrm{s}\right.$; cop. ca. 1400] ACoutBordB 40[32]; am biafora et am crit [13 ${ }^{\mathrm{e}}$ s.; cop. ca. 1400] ACoutBordB 59[55], bia fora [av. 1300; cop. 1489] CoutBazasB, ArchGir 15,100[41: cridar bia fora];147[175: auer bia fora], biefore: cridar biefore 1268 AnglGuyB 159b[415] (v. ci-dessous sub 3).

FEW VIA 14,377b: abéarn. biafore «cri par lequel ceux qui ont vu commettre un crime appellent le peuple pour poursuivre le criminel» $13^{\mathrm{e}} \mathrm{s}$.Laur 1704, aussi DC, biahores Rag 1583, Gers biahoro «au meurtre» (vieux), Lavedan biahore «cri d'alarme pour appeler au secours»; Lv 8, 748a/b: abéarn. biaffora m. «Hilferuf» ca. 1400 ForsB [= LR sub criit]; m. et f. 1304 Bayonne, EtR 2,87; ZrP 35,459-469 (avec d'autres attestations après 1300). Cf. aland. (cridar) biaffore m. «au secours, cri d'alarme» ca. 1468 CoutDax, ArchGir 37,68[264];70-71[273-278] et abéarn. criit et biaffora ca. 1400 ForsB[OG]Gloss «cri et appel au secours; procédure de flagrant délit».

$\diamond$ Rem. Étymologiquement, bihore remonte a via fora et désigne donc un cri qui invite à sortir. Le cri de biafore obligeait les habitants d'un lieu à sortir en armes de leurs maisons pour prêter main-forte à la victime ou aux autorités.

2. «à l'aide» (renforçant biafora)

(biafora!) ajuda! [13 s.; cop. ca. 1400] ACoutBordB 40[32].

ø FEW ADJUTARE 24,162b; LR sub biahore: abéarn. Biaffora, ajuda! «au secours, aide!» $15^{\mathrm{e}} \mathrm{s}$. BaronBéarn.

3. «levée de gens, appel au secours/aux armes, fait au son des cloches ou par tout autre signal d'alarme, en cas de danger public (pour poursuivre ou repousser des ennemis ou des voleurs)»

cridar orde (e biefore) synt.verb. 1268 AnglGuyB 159b[415: cridar ord $[r] e]$.

À rattacher à FEW QUIRITARE 2,1484b/1485a et ORDO 7,407a; Lv 5,511b: aland. orde 1351 CartDax, LNoirDax, agasc. far oorde 1313 Lavedan; v. le comment. dans LR sub orde.

$\diamond$ Rem. V. ForsB[OG] p. 163 n1 et gloss p. 692: ordre et biafore (qui équivaut à criit et biaffore) désigne le droit de poursuite d'un criminel avec l'aide de la population.

1 biaffora, (auer) bia fora

1238 [cop. $14^{\mathrm{e}} \mathrm{s}$.] Entre-deux-Mers: Item, compellissan li homes de la gleysa et dels cavaleys a ffar os sino a audit lo crit de Biaffora contre l'assaut o la present rapina. - [av. 1300; cop. 1489] Bazas: si una persona a . . f feyt aucun crim en al- 
cuna senhoria, et es escapa en un autra on es pres, et lo seguen an la court et an bia fora, que lo baile ... no lo remetra point a l'autre, entroque aia feyt enformacion a totz querelhans (cridar biafora v. ci-dessous 2; cridar biefore v. ci-dessous 3).

\section{2 ajuda!}

[13 ${ }^{\mathrm{e}}$ s.; cop. ca. 1400] Bordeaux: Costuma et ussatge es en la vila de Bordeu que nulhs homs no s'pot apperar barreyat ni raubat de jorns; e la rasson es quar de jorns tot lo mont ac pot veder, et pot hom cridar: «Biafora! Ajuda!».

3 cridar orde

1268 Guyenne: qui aubergatz fera forceirement, autres .XX. s., si no cride ord $[r] e$ e biefore lore que aubergatz sera.

\section{9 protéger, défendre qn, qch:}

1. «protéger (qn), prendre (qn) sous sa protection, donner appui à (qn)» amparar (qn) v.tr. 1171 [cop. 1251] Bagnères-de-Bigorre, RecGascL 22; 1260 CoutBagn, MusArch 169; RecGascL 34.

FEW PARARE 7,631b: < anteparare d'où apr. antparar «protéger» SFoy, amparar «protéger» Rn; Lv; etc.

2. emparar (qn, qch) v.tr. 1125 [cop. ca. 1390] CartBigRC 63[44,8;10: ms. Pa]; [ca. 1175; cop. ca. 1390] CartBigRC 67[46,4];68[49,7: ms. Pa]; 1256 [cop. ca. 1320] CartBlanc, CartAuchL 297; RecGascL $105=$ Lv 2,377a sub 1; 1259 [cop. 14 s.] LOrB 33; [1273] ACoutBay, Bal 2,621[71]; 1283 [cop. 1336] EtBay 253 [294]; [13 s.; cop.] CoutMeilhanB, ArchGir 25,142[24;27], enparar 1125 [cop. ca. 1250] CartBigRC [44,9: var. B].

FEW PARARE 7,631b/632a: < *imparare d'où apr. emparar v.a. «protéger; ... » [avec n65] dp. ca. 1200, surtout Toulouse, gasc., aland. «protéger; soutenir; occuper» [= MillGloss: «protéger, prendre sous sa protection» 1306 MMarsan], Puiss. emparâ «défendre, protéger», béarn. «protéger, garantir».

3. defener (qn) v.tr. 1171 [cop. 1251] Bagnères-de-Bigorre, RecGascL 22; 1278 [cop. 1551] CartOloron, ForOlDC 106a[5] = LR sub defene «défendre», deffener (qn, qch) [12 $\mathrm{s} . ;$ cop. ca. 1460] ForGén, ForsB[OG] 162[36]; deffener (qch) [12 s.; cop. ca. 1460] ForGén, ForsB[OG] 162[37]; 1247 [cop. ca. 1460] ForAspe, ForsB[OG] 532[3]; 1259 [cop. 14 s.] LOrB 33; 1267 [cop. ca. 1460] ForOss, ForsB[OG] 520[11]; 1278 [cop. ca. 1460] ForOlDC 107a[5], defendre (qn) 1270 [cop. 1380] ChartSSeverPC 478.

FEW DEFENDERE 3,28b: apr. defendre «protéger», aland. defener «prendre des mesures de défense», béarn. defende «défendre»; cf. aussi RecGascLGloss defener 1272 Bayonne [source non vérifiable]. 
4. gardar (qn, qch) v.tr. 1255 [cop. 1341] CoutMontrOG 142[49], guardar 1275 Bordeaux [concern. Salles et Mios], ArchGir 25,490; [ca. 1290] HommVayresD, ArchGir 8,42.

FEW germ. *WARDON 17,514a: apr. gardar v.a. «avoir l'œil sur, soit pour protéger, soit pour empêcher de nuire»; LR sub goarda: abéarn. gardar (qn) «garder (qn)» ca. 1330 [cop. ca. 1410] HistSainte.

5. prender (qn) a sa segurtad synt.verb. 1249 [n.st.] LaRéole, ArchGir 15,168[9].

À rattacher à FEW PREHENDERE 9,340 et SECURUS 11,390a: apr. prendre en sa segurtad «prendre sous sa protection» $1248[=\mathrm{Lv} 7,532 \mathrm{a}$ sub 4 «in seinen Schutz nehmen»: alang. prendre e nostra segurtad ChartAgen].

6. «protéger (qn) contre, prendre la défense de (qn)»

a) amparar (qn) de (qn) v.tr. [ca. 1270; cop. ca. 1390] CartBigRC 112[78,6/7; 79,3/4].

$ø$ FEW PARARE 7,631b.

b) emparar (qn) de (qn) v.tr. 1125 [cop. ca. 1390] CartBigRC 63[44,38: ms. Pa]; [1207; cop. 16 ${ }^{\mathrm{e}}$ s.] CoutLaRéoleG, ArchGir 2,264[43]; 1250 [cop. 1274] Orthez, ArchGir 2,304; 1256 Casteljaloux, RecGascL 79; emparar (qn) de (qch) [ca. 1175; cop. ca. 1390] CartBigRC 98[68,5].

ø FEW PARARE 7,631b/632a.

c) defener (qn) de (qn) v.tr. 1207 [cop. 16e s.] CoutLaRéoleG, ArchGir 2,264 [43], deffender (qn) de (qn, qch) 1270 TestAman VI d'Albret, RecGascL 95; [13 ${ }^{\mathrm{e}}$ s.; cop.] CoutMeilhanB, ArchGir 25,143[29], defendre (qn) de (qn, qch) 1250 [cop. 1274] Orthez, ArchGir 2,304; 1278 [n.st.] Bordeaux, ArchGir 43,171 [164].

ø FEW DEFENDERE 3,28b; Rn 4,360b: apr. defendre (qn) de (qn) [av. 1400] PBussignac.

7. «défendre sa personne ou ses biens» [v. aussi sub protéger, garder, préserver $2662,4 \mathrm{a} ; \mathrm{b}]$

defener soo cos o sas causas synt.verb. 1260 CoutBagn, MusArch 168; RecGascL 34 avec n1.

À rattacher à FEW DEFENDERE 3,28b, CORPUS 2,1214a et CAUSA 2,541b.

8. «défendre (qn) selon le droit»

defene $[\boldsymbol{r}]$ adreit (qn) loc.verb. 1256 [cop. ca. 1320] CartBlanc, CartAuchL 297; RecGascL 105 [d. a dreit].

À rattacher à FEW DEFENDERE 3,28b et DIRECTUS 3,89b; Rn 5,74b: apr. qui tort adreg defen ca. 1270 BertZorzi. 
9. «mettre en sécurité, protéger (qn), sauver (la vie de qn)»

saubar (son cos) v.tr. 1252 [cop.?] Soule, RecGascL 48[27; gloss «sauver, garantir»].

FEW SALVARE 11,128a: apr. salvar «tirer hors de péril, mettre en sûreté», béarn. saubá; Rn 5,144a: apr. son cors salvar «sauver son corps» ca. 1235 PCard.

10. «protéger (un criminel), mettre à l'abri, accueillir (qn)» emparar (qn) v.tr. 1291 [cop. 1336?] EtBay 91[93].

ø FEW PARARE 7,632a; cf. ib.,631b: apr. amparar «accueillir» Lv [2,379a sub 10 «freundlich empfangen»: aussi alang. emparar $14^{\mathrm{e}}$ s. GestaKaroli].

11. «protéger, défendre (un château, une ville)»

a) deffendre v.tr., deffende [r] [ca. 1290] HommVayresD, ArchGir 8,42.

FEW DEFENDERE 3,28b: aland. defener «prendre des mesures de défense».

b) prener en emparansa (la biela de) synt.verb. [ca. 1175; cop. ca. 1390] CartBigRC 98[68,2: ms. Pa], prener [en] emparanssa [ca. 1175; cop. après 1425] CartBigRC [68,2: var. Pb].

À rattacher à FEW PREHENDERE 9,340 etc. et PARARE 7,632b; Lv 2,376b sub 1 «in Schutz nehmen»: alang. prendre en amparansa ca. 1350 LeysAm.

1 amparar (qn)

1260 Arné (Bagnères-de-Bigorre): si nulh home mete ni amparave nulh autre qui tort sabud agos a nulh bezii de Banheres ...

2 emparar (qn)

1125 [cop. ca. 1390] Bigorre: Lo coms de Begorra empara Sans Gassie (v. aussi ci-dessous 8).

3 defener (qn)

1171 [cop. 1251] Bagnères-de-Bigorre: que si nulh home i benia per bezin ester ... e-1 senhor defen et.

4 gardar (qn, qch)

1255 [cop. 1341] Montréal-du-Gers: juron sober sentz evangelis . . que gardaran son cors e sa vita e sa senhoria ...

5 prender (qn) a sa segurtad

1249 [n.st.] LaRéole: fam assaber que nos prendem a nostra segurtad e a nostra garda e a nostra protection totz los homes e las gens de tota la terra de l' senhor de Toloza.

6a $\operatorname{amparar}$ (qn) de (qn)

[ca. 1270; cop. ca. 1390] Lourdes: amparar de (v. sub protéger, garder, préserver $2662,1)$. 
6b emparar (qn) de (qch)

[ca. 1175; cop. ca. 1390] Bigorre: E nos e-ls nostres que-ls deuem emparar de tota penhera de senhor.

$6 \mathrm{c}$ deffender (qn) de (qch)

1270 Casteljaloux: . . . que ed recepia en sin la qarqa de la tuteiria de mos enfants e de ma terra ... E que-ls deffenda de pleitz et de guerras que a en auant sa poiren escazer.

7 defener soo cos o sas causas

1260 Arné (Bagnères-de-Bigorre): que nulh bezii de Banheres aucigos nulh autre soo bezii de Banheres maa irade, solemenz que defene $[n]$ [RecGascL corrige: defenere «défendrait»] soo cos o sas causas que hom lo toros malignemenz.

8 defene $[r]$ adreit (qn)

1256 [cop. ca. 1320] Auch: si per abenture mos erez ne mos parentz . . . ne contradizen a la dite donation e que no poguoc aver valor ne tengude ne ac poguosen emparar ne defene adreit los ditz canonies, io Guiraud dit d'Archamont donc aus ditz canonies M sols de bos morlas.

9 saubar (son cos)

1252 [cop.?] Soule: saubas (v. sub donner sauf-conduit 2672,2).

10 emparar (qn)

1291 [cop. 1336?] Bayonne: que nulhs hom o femne estrainh ni priuat no empari ni tinque murtrer ni banitz.

11a deffendre

[ca. 1290] Vayres [Gir.]: lo senher de Bayras a tau senhoria . . . mande a lor, o aujan [subj.prés. de audir] lo corn deu castet de Bayras, et deuen ja benir, ab armas, et entrar en castet, et deffendre a lur poder.

$11 \mathrm{~b}$ prener en emparansa

[ca. 1175; cop. ca. 1390] Louey (Ossun): que Centod, coms de Begorre, prenco en emparansa la biela de Loey a testimoni de la cort de Begorra.

\section{0 défense, protection:}

1. «protection, défense militaire»

defensa f. 1171 [cop. 1251] Bagnères-de-Bigorre, RecGascL 21.

FEW DEFENSA 3,29a: apr. defe( $n$ )sa «défense» [= LvP «défense, protection»].

2. «protection (fournie par une autorité à qn)»

protection f. 1241 DocSEm, ArchGir 28,494; 1249 [n.st.] LaRéole, ArchGir 15, 168[9], protectio [1270; cop. 1605] PrivGavarnie, TextBigM 94[12]. 
ø FEW PROTEGERE 9,470a: apr. protection $\mathrm{f}$. «action de préserver qn de mal» 1425, Pans; Rn 5,312a: protectio e salvagarda 1241, Doat [alang ou agasc.?]; LR: béarn. proutectiou «protection».

$\diamond$ Rem. L'acception est très proche et liée à la notion «défense, sauvegarde, surtout de la part du roi ou d'un seigneur féodal».

3. «défense, protection (d'un lieu, d'une communauté religieuse)»

emparansa f. [ca. 1175; cop. ca. 1390] CartBig v. prener en emparansa sub protéger, défendre (qn, qch) 2659,11b, emparanssa [ca. 1175; cop. après 1425] CartBig v. prener [en] emparanssa sub 2659,11b, emparance 1277 [cop.] Mill, ArchGir 45,69[28].

FEW PARARE 7,632b: agasc. emparansa f. «protection», abéarn. emparance [= LR «protection, garantie, préservation» (source non indiquée)].

4. «délai de protection (accordé par le seigneur à qn et ses biens)»

respit $\mathrm{m}$. [13 ${ }^{\mathrm{e}} \mathrm{s}$; cop.] CoutMeilhanB, ArchGir 25,142[22].

ø FEW RESPECTUS 10,306a.

1 defensa

1171 [cop. 1251] Bagnères-de-Bigorre: . . . asi cum en queste carte es escriut, per so que-1 senhor e tote la terre i trobas cosselh e defensa.

2 protection

1241 SÉmilion: que dauantdeitz bescomz a receubo etz et cadaun en per si et totas las lurs causas moblas et no moblas, causque sian, uniuersaument en son saup conduit et en sa protection (protectio v. sub sauvegarde 2667,1).

3 emparance

1277 [cop.] Loubens: artiem nos . . cum a senhor temporau, la garde e la emparance de la dite maizon [= maison de ordi de dones].

4 respit

[13 ${ }^{\mathrm{e}}$ s.; cop.] Meilhan-sur-Garonne: que si nulhs hom de la vila se vol mudar en altra vila, que-1 deu dar lo senhor dia respit per XL. dies, e tenir luy e ses causas saubes e segures de tot son poder.

\section{1 défenseur, protecteur, soutien:}

1. «personne qui aide à protéger et défendre les intérêts (de qn, ici des mineurs)»

defendedor m. 1270 TestAman VI d'Albret, RecGascL 96.

FEW DEFENDERE 3,28b: apr. defendedor «défenseur»; LvP «id.; tuteur».

1 defendedor

1270 Casteljaloux: E doni al tutor, cosselhedors e ajudedors e defendedors. 


\section{2 protéger, garder, préserver:}

1. «protéger, préserver (qn) de (qch)» gardar (qn) de (mal, mau, daun, tort, etc.) v.tr. [ca. 1114; cop. ca. 1390] CartBigRC 22[7,24: ms. Pa]; [ca. 1270; cop. après 1425] CartBigRC 112[78,7;79,4: ms. Pb]; 1277 [cop. $15^{\mathrm{e}}$ s.] CartOssauT 53; 1285 Tarbes, RecGascL 40, guoardar $\boldsymbol{d e}$ [1273] ACoutBay, Bal 2,596[3,1], guardar de [ca. 1114; cop. ca. 1250] CartBigRC [7,25: var. B]; 1278 [n.st.] Bordeaux, ArchGir 43,171[164].

ø FEW germ. *WARDON 17,514; Lv 4,54a sub 2 «bewahren, behüten, schützen»: apr. guardar de GuillMont ca. 1245, etc.; LR sub goarda: abéarn. gardar (qn) de (qn) «protéger (qn) contre (qn)» ca. 1330 [cop. ca. 1410] HistSainte.

2. guarir (qn) de (daun, greu) v.tr. 1246 Orthez, RecGascL 47[26].

FEW abfrq. got. *WARJAN 17,526a: apr. guarir v.a. «préserver; sauver; etc.», garir «id.; garantir; etc.»; Rn 3,431a: apr. guerir (qn) de mort «préserver (qn) de mort» ca. 1190 RichBerbezill.

3. «protéger, garantir (qn, qch) de (dommage)»

treger (qch) de (dommage) v.tr. [1252; cop. ca. 1460] ForGén, ForsB[OG] 186 [76, gloss ,garantir, dégager de“"].

$\varnothing$ FEW TRAHERE $13^{2}, 177 \mathrm{~b}$; cf. LR sub trege: (a)béarn. treger (qn) de (servitude, danger) «tirer (qn) de» ca. 1330 [cop. ca. 1410] HistSainte; 1583 Salette.

4. «protéger (une personne et sa propriété)» [v. aussi sub protéger, défendre qn, qch 2659,7]

a) tenir (luy e ses causas) saubes e segures loc.verb. [13 ${ }^{\mathrm{e}}$ s.; cop.] CoutMeilhanB, ArchGir 25,142[22].

À rattacher à FEW TENERE $13^{1}, 210 \mathrm{a}$, SALVUS 11,134a et SECURUS 11, 388a; LR sub saubament adv.: tier (qch) saubament e segura «tenir (qch) en sauvegarde et sûrement» [s.d.] ArchBPyr.

b) thier (les causes) saubes et segures (los ungs aus autres) loc.verb. 1292 [cop. ca. 1480] CartDax: LNoirDaxA, ArchGir 37,275, thier saubes e segures (los uns [aus] autres) 1292 [cop. ca. 1390] CartDax: LNoirDaxA, ArchGir 37, 275 [var.].

À rattacher à FEW TENERE $13^{1}, 210 \mathrm{a}$, SALVUS 11,134a et SECURUS 11, 388a.

5. «se protéger réciproquement, se porter garant de (qch) (en parl. de la personne et de ses biens)»

se saubar (los ungs aus autres los cors et totes les causes) v.pron. 1292 [cop. ca. 1480] CartDax: LNoirDaxA, ArchGir 37,273, gloss «se réserver, se ga- 
rantir» = Lv 7,449a sub 1 „,nicht verletzen, nicht beschädigen“? ou „,die Sicherheit verbürgen"?

$ø$ FEW SALVARE $11,128$.

1 gardar (qn) de (qch)

[ca. 1270; cop. après 1425] Lourdes: que Do de Sene . . . deu dar IIII. d. totz ans . . . per ces en tote sa bite e lo segnor de Lorde que-u deu amparar a so poder de totz homes et gardar de mau.

2 guarir (qn) de (qch)

1246 Orthez: . . . que de tot daun et de tot greu los ne guarin sober nos e sober totas nostres causas ... E nos comtessa e-n Gaston, autreiam vos bonement que . . bon guarem de daun e de destarz.

3 treger (qch) de

[1252; cop. ca. 1460] Béarn: De eodem establiment: de treger la fidance de dampnadge.

4a tenir (luy e ses causas) saubes e segures

[13 $\mathrm{s}$.; cop.] Meilhan-sur-Garonne: tenir (luy e ses causas) saubes e segures (v. sub défense, protection 2660,4).

$4 \mathrm{~b}$ thier (les causes) saubes et segures (los ungs aus autres)

1292 [cop. ca. 1480] Tartas: .. . les causes que thiencossen saubes et segures los ungs aus autres per XL dies deffens los quoaus ac pogossen trer saubementz.

\section{3 protection, préservation:}

1. defensio[n] f.: defensios pl. 1270 Mill, ArchGir 45,7[9].

FEW DEFENSIO 3,29a: apr. defension «défense».

\section{1 defensio $[n]$}

1270 MMarsan: Auant los prometem portar e far bones e fermes garenties e defensios de les auant dites causes, e de totz lors dretayges, de tots homes e de totes femnes, sos obligation de tots nostres bes.

\section{4 protéger, garantir qch:}

1. amparar (fors, costumes) v.tr. 1171 [cop. 1251] Bagnères-de-Bigorre, RecGascL 27.

FEW PARARE 7,631b: apr. amparar «. . .; protéger; . . .».

2. emparar (carte) v.tr. 1256 [n.st.; cop. ca. 1460] ForGén, ForsB[OG] 210[124], gloss «protéger, maintenir en son droit, garantir». 
ø FEW PARARE 7,631b/632a: alyon. empara «défendre de, garantir de» $16^{\mathrm{e}}$ s., béarn. emparâ «protéger, garantir»; LR sub empara: emparar «garantir (un droit)» 1676 PrivRègl.

\section{1 amparar}

1171 [cop. 1251] Bagnères-de-Bigorre: dam e autrejam los auant diitz fors e costumes ester servades e tengudes e amparades per nos e per totz los nostres successors.

2 emparar

1256 [n.st.; cop. ca. 1460] Béarn: aquere carte qui feyte es ni se fara sie tengude, saubade et emparade et aye integre valor tot clarementz aixi cum la carte disera ni mustrara.

\section{5 protecteur, garant:}

1. amparai m. [ca. 1185] Comminges, Brunel 220[227,23/24].

FEW PARARE 7,631b: apr. [à corr. agasc.] amparai avec n64: ,Nom.sg., also für -aire' «protecteur» Comminges 1185, Brunel [v. l'attestation cidessus]; LR: abéarn. emparador «qui protège, garantit, préserve» [sans indication de source].

1 amparai

[ca. 1185] Comminges: ... e deu n'este bos guarenz e bos amparai de omnibus ominibus.

\section{6 garde, protection:}

1. garde f. 1277 [cop.] Mill, ArchGir 45,69[28].

FEW germ. *WARDON 17,515b: apr. garda f. «surveillance, protection, soin qu'on prend de qch» Rn; Lv; BrunelS.

2. «(tenir une place, un lieu) en garde de la part de (qn, ici le seigneur dans le but de ne pas la/le livrer à son ennemi)»

en guarda de loc.prép. [13 ${ }^{\mathrm{e}}$ s.; cop. ca. 1400] ACoutBordB 37[27].

À rattacher à FEW germ. *WARDON 17,515b; cf. Rn 3,425a: apr. en garda de «en garde de» ca. 1170 Codi.

1 garde

1277 [cop.] Loubens: .. . la garde e la emparance de la dite maizon, . .., de totes las causes que nos hi auem donades.

2 en guarda de

[13 ${ }^{\mathrm{e}}$ s.; cop. ca. 1400] Bordeaux: totz homs pert sa franquessa ... per aquestas 
causas: quant hom ... livra castetz o bila, quant la ten en guarda deu senhor, et... la livra a sons enamicx.

\section{7 sauvegarde:}

1. «protection accordée par une autorité»

sauba garda f. [1270; cop. ca. 1590] PrivGavarnie, TextBigM 94[12: var.]; [1284; cop. ca. 1590] PrivGavarnie, TextBigM 93[11: var.], salvagarda 1294 [cop. 1343] CoutLect, ArchLectD 40[37];52[86], sauuagarde [1270; cop. ca. 1600] PrivGavarnie, TextBigM 94[12]; [1284; cop. ca. 1600] PrivGavarnie, TextBigM 93[11], saubegarde [1270; cop. $19^{\mathrm{e}} \mathrm{s}$.] PrivGavarnie, TextBigM 94[12: var.]; [1284; cop. $19^{\mathrm{e}}$ s.] PrivGavarnie, TextBigM 93[11: var.].

$\varnothing$ FEW SALVUS 11,134a et germ. *WARDON 17,516b; Rn 3,429a: salvagarda 1294, Doat [alang. ou agasc.?]; LR sub saubagarde et tuition: abéarn. saubegarde $15^{\mathrm{e}} \mathrm{s}$. BaronBéarn, ib. sub brisadou: agasc. id. [s.d.] Bayonne; sub soul: béarn. sauvagoarda 1583 Salette. Cf. aussi aland. saubegarde «sauvegarde» ca. 1468 CoutDax, ArchGir 37,74[291].

2. «protection, sauvegarde»

segurtat $\mathrm{m}$. [13 ${ }^{\mathrm{e}}$ s.; cop. ca. 1400] ACoutBordB 29[13].

FEW SECURUS 11,390a: béarn. seguretàt «sûreté, sécurité», seguritàt; apr. prendre en sa segurtad "prendre sous sa protection» $1248[=\mathrm{Lv} 7$, 532a sub 4 «in seinen Schutz nehmen»: alang. ChartAgen]; Rn 5,184b: aprov. letras de segurtat «lettres de sûreté [lettre par laquelle est accordée un sauf-conduit]» ca. 1300 SHon.

\section{1 saunagarde}

[1270; cop. ca. 1600] Gavarnie: lo Rey don [Jayme] d arago . . meto en sauuagarde et protectio per toutes sas seignories ... los habitans.

\section{2 segurtat}

[13 ${ }^{\text {e }}$ s.; cop. ca. 1400] Bordeaux: ssi lo baten ... e es proat . . sera condempnat a una soma d'argent ...; et, si demanda segurtat, lo mager et los juratz ly deven dar seguranssa.

\section{8 sauf, sauvegardé:}

1. «sauf (en parl. d'une pers.)» saub adj. 1267 [cop. ca. 1460] ForOss, ForsB[OG] 516[5;6].

FEW SALVUS 11,133b/134a: apr. salv, sal adj. «qui n'a pas reçu d'atteinte, qui a échappé au péril» AimBel, béarn. saub. 
2. «sûr (en parl. d'un chemin)»

saub adj. [12 s.; cop. ca. 1460] ForGén, ForsB[OG] 162[37] = LR: (camiis) saubs pl. «(chemins) sûrs, où l'on est en sûreté».

$\varnothing$ FEW SALVUS 11,133b/134a.

$1 \mathrm{saub}$

1267 [cop. ca. 1460] Ossau: tot hom qui pusque atenher a la senhorie d'Ossau deu esser saub.

2 saub

[12 s.; cop. ca. 1460] Béarn: Que tenguen los camiis saubs et nulh franc hom no y lexen penherar ni marcar.

\section{9 en sauvegarde et en sécurité:}

1. «sain et sauf (personne et/ou biens)»

(anar, bier) saub et segur loc.adv.: anar saub et segur [1220; cop. ca. 1460] ForMorl, ForsB[OG] 328[32]; bier saub et segur davant (qn) 1267 [cop. ca. 1460] ForOss, ForsB[OG] 518[9]; 1292 [cop. ca. 1480] CartDax v. thier (qch) saub et segur sub protéger, garder, préserver 2662,4b, saub e segur: far miar l'aver saub e-u coos segur 1278 [cop. ca. 1460] ForOlDC 105a[4: var. l'aver saub e-u cors segur [cop. 1551], CartOloron, ForOlDC 104a(4)]; 1292 [cop. ca. 1390] CartDax v. thier (qch) saub e segur sub 2662,4b; [13 ${ }^{\mathrm{e}} \mathrm{s}$; cop.] CoutMeilhan v. tenir (qn, qch) saub e segur sub 2662,4a.

À rattacher à FEW SALVUS 11,134a et SECURUS 11,388a; Rn 5,145a: apr. s'en anar sas e sals «sain et sauf» ca. 1300 EvNicod, s'en tornar san e salv ca. 1300 SHon; cf. LR sub saubament adv.: tier (qch) saubament e segura «tenir (qch) en sauvegarde et sûrement» [s.d.] ArchBPyr.

2. «être sous sauvegarde, être protégé (personne et/ou biens)» (esser) saub et segur loc.adj. [1252; cop. ca. 1460] ForGén, ForsB[OG] 182[71], saub e segur [13 ${ }^{\mathrm{e}}$ s.; cop.] CoutMeilhanB, ArchGir 25,141[12]; en saub et en segur [1220; cop. ca. 1460] ForMorl, ForsB[OG] 314[8].

À rattacher à FEW SALVUS 11,134a et SECURUS 11,388a; Lv 7,446b sub 3 «ungefährdet, sicher»: alang. (esser) salp e segur de (faire qch) 1391 Axsur-Ariège.

3. «en sécurité, sain et sauf»

saubementz adv. 1292 [cop. ca. 1480] CartDax: LNoirDaxA, ArchGir 37,275 [gloss «sans empêchement»].

FEW SALVUS 11,134a: abéarn. saubament adv. «en sécurité, hors de danger»; Lv 7,448b/449a: apr. salvamen «en sûreté» [LvP] ca. 1170 PAlv [Rn 5,145a «salutairement», corr. par Lv en «wohlbehalten, unverletzt?» ou 
«sicher, ungefährdet?»]; LR: abéarn. saubament e segura «en sauvegarde et sûrement» [s.d.] ArchBPyr. Cf. aussi agasc. saubament et segurament «la vie sauve et en sécurité»1386 Langon, ArchGir 3,279.

1 (anar) saub et segur

[1220; cop. ca. 1460] Morlaàs: la [à corr. lo] senhor lo deu dar guide per tote sa terre entro que eg sie en loc de saubetat; et que ani saub et segur (saub (l'aver) $e$-u (cors) segur v. sub conduire, guider 2673,2).

2 (esser) saub et segur

[1252; cop. ca. 1460] Béarn: egs et totas lors causes sien saubes et segures ayxi cum si a cortz eren manatz (saub e segur v. sub défense, protection 2660,4; en saub et en segur v. sub sauf-conduit 2671,2).

3 saubementz

1292 [cop. ca. 1480] Tartas: saubementz (v. protéger, garder, préserver 2662,4b).

\section{0 sauf et sûr (d'un lieu):}

1. (carrere, loc) saub et segur loc.adj. [1220; cop. ca. 1460] ForMorl, ForsB[OG] 310 [2];314[8]; (loc) saub e segur [13 ${ }^{\mathrm{e}}$ s.; cop.] CoutMeilhanB, ArchGir 25,142 [24].

À rattacher à FEW SALVUS 11,134a et SECURUS 11,388a.

1 (carrere) saub et segur

[1220; cop. ca. 1460] Morlaàs: aqueg qui a-ffe no deu dar au senhor nulh daun, si no que la tort fos feyt en la carrere afforade, car aquere deu estar saube et segure.

\section{1 sauf-conduit:}

1. «sauf-conduit, laissez-passer, protection»

saup conduit loc.m. 1241 DocSEm, ArchGir 28,494.

FEW CONDUCERE 2,1026a: apr. salvconduch «permission d'aller dans un lieu et d'en revenir sans être arrêté» 1385, salcondug Millau 1363, Doc 135; LR: abéarn. saup-condut «sauf-conduit» [s.d.] ArchBPyr, ib. sub segurtance: $i d$. ca. 1490 LRougeOssau.

2. guiit m. [1220; cop. ca. 1460] ForMorl, ForsB[OG] 314[8].

FEW abfrq. *WITAN 17,601b: apr. guit $\mathrm{m}$. «action de conduire, de diriger» $13^{\mathrm{e}} \mathrm{s}$; cf. LR sub guidoadge: abéarn. guiit «droit de sauf-conduit (du bétail)» ca. 1490 LRougeOssau.

$\diamond$ Rem. «Le mot guiit désigne le sauf-conduit donné par le seigneur», ForsB[OG] $317 \mathrm{n} 2$. 
3. guide f. [1220; cop. ca. 1460] ForMorl v. dar guide sub donner sauf-conduit $2672,4 \mathrm{a}$.

ø FEW abfrq. *WITAN 17,601b/602a; cf. Lv 4,209b sub 2: agasc. aber guida «sicheres Geleit?»14 $\mathrm{s}$. CoutGont.

4. guidoo m. [1220; cop. $15^{\mathrm{e}} \mathrm{s}$.] ForMorl v. dar guidoo sub donner sauf-conduit $2672,4 b$, guido ca. 1233 ChartAgenMT 1,33 = Lv.

FEW abfrq. *WITAN 17,602b: apr. guidó m. «sauf-conduit» Albi 1233; Lv 4,212a sub guidon 3 «sicheres Geleit, Sicherheit»: agasc. guidon $14^{\mathrm{e}} \mathrm{s}$. CoutGont.

5. guidoadge m. 1171 [cop. 1251] Bagnères-de-Bigorre v. amenar en son guidoadge sub donner sauf-conduit 2672,5.

FEW abfrq. *WITAN 17,603a: apr. guidoadge m. «sauf-conduit» gasc. 1251 [v. l'attestation ci-dessus].

6. saup anar e saub venir loc.m. 1265 [cop. 14e s.] PrivMonségur: CartMonségurM, ArchGir 5,6.

À rattacher à FEW *AMBITARE 24,400a, VENIRE 14,241b et SALVUS 11, 134b: cf. afr. mfr. sauf alant et sauf venant $\mathrm{m}$. «sauf-conduit» $13^{\mathrm{e}} \mathrm{s}$.-Froiss; cf. Lv 7,446b sub 3 «ungefährdet, sicher»: alang. (qn) sia salp e segur de anar e retournar 1391 Ax-sur-Ariège.

1 saup conduit

1241 SÉmilion: saup conduit (v. sub défense, protection 2660,2).

2 guiit

[1220; cop. ca. 1460] Morlaàs: [de choses prêtées d'un étranger, ennemi du seigneur qui sont à rendre au prêteur] . . . empero que en saub et en segur lo sien las causes ab guiit deu senhor entro que en loc saub et segur sien.

6 saup anar e saub venir

1265 [cop. $14^{\mathrm{e}} \mathrm{s}$.] Monségur: E deven aver mercat al dimercles, saup anar e saub venir, a tot home que hi venga, si pero no y ave mort home o plagat o pres.

\section{2 donner sauf-conduit:}

1. «accompagner (qn) de sa protection»

segui $[\boldsymbol{r}]$ (qn) v.tr. 1278 ForOlDC 104b; far seguir (qn) loc.verb. 1278 [cop. ca. 1460] ForOl, ForsB[OG] 498[3].

$ø$ FEW SEQUI $11,488 b$ etc.

2. far segurtad de son cos synt.verb. 1252 [cop.?] Soule, RecGascL 48[27]. 
À rattacher à FEW CORPUS 2,1214a et SECURUS 11,390a; cf. ib.,390b: apr. letras de segurtat «sauf-conduit» ca. 1290 [= Rn 5,184b: SHon].

3. guidar (qn) v.tr. 1171 [cop. 1251] Bagnères-de-Bigorre, RecGascL 22;25 «conduire, donner un sauf-conduit» $=$ Lv 4,213a sub guidonatge 1, ib. $210 \mathrm{~b}$ sub guidar 5; [13 ${ }^{\mathrm{e}}$ s.; cop.] CoutMeilhanB, ArchGir 25,141[16].

FEW abfrq. *WITAN 17,601b: apr. guidar v.a. «accompagner (qn) pour le protéger; donner un sauf-conduit (à qn)» gasc. $13^{\mathrm{e}} \mathrm{s}$.; etc.; Lv 4,211b sub 5 «sicheres Geleit gewähren, Schutz gewähren, schützen»: agasc. guidar $14^{\mathrm{e}} \mathrm{s}$. CoutGont, guizar 1303 CoutPouy-Carréjelart.

4. «donner guidage»

a) dar guide loc.verb. [1220; cop. ca. 1460] ForMorl, ForsB[OG] 328[32].

À rattacher à FEW DARE 3,14b et abfrq. *WITAN 17,601b/602a.

b) dar guidoo loc.verb. [1220; cop. $15^{\mathrm{e}}$ s.] ForMorl, ForsB[OG] 328[32: var.].

À rattacher à FEW DARE 3,14b et abfrq. *WITAN 17,602b.

5. amenar en son guidoadge synt.verb. 1171 [cop. 1251] Bagnères-de-Bigorre, RecGascL 22 = Lv 4,213a sub guidonatge 1 «zur Sicherheit dienende Begleitung, sicheres Geleit, Schutz».

À rattacher à FEW MINARE $6^{2}, 106$ b et abfrq. *WITAN 17,603a.

1 segui $[r]$ (qn)

1278 Oloron: lo diit En Fortaner que-us deu saubar e segui enta que part se boilhen anadere de I die.

2 far segurtad de son cos

1252 [cop.?] Soule: R.W. Vescoms de Soula . . trameto assa cort .I. caver . . qui mostras per lueys aus [sic] coms Simon que apareilad ere d'en cort anar en tot loc ..., solemenz que le coms lo fes segurtad de son cos qu'en saubas.

3 guidar (qn)

[13 ${ }^{\mathrm{e}}$ s.; cop.] Meilhan-sur-Garonne: E establi que ja lo senhor homes no guides qui tort agos en la vila, si no eg fazia per voluntat de celuy a cuy auria lo tort.

4 a dar guide

[1220; cop. ca. 1460] Morlaàs: Si augun homi d'esta biele s'en vol anar . . . la [sic] senhor lo deu dar guide [var. guidoo] per tote sa terre entro que eg sie en loc de saubetat.

$4 \mathrm{~b}$ dar guidoo

[1220; cop. $15^{\mathrm{e}}$ s.] Morlaàs: dar guidoo (v. ci-dessus 4a, var.). 


\section{3 conduire, guider:}

1. «guider, accompagner (qn)»

guidar (qn) v.tr. [ca. 1190] ChMalf, Bal 1,423; 1300 [cop. 1466] CoutCastBouz, Monl 6,104.

FEW abfrq. *WITAN 17,601b: apr. guidar v.a. «conduire» SFoy; etc.

2. «mener, conduire (qn)»

miar (qn) v.tr. 1278 [cop. ca. 1460] ForOlDC 105a[4], menar 1294 [cop. 1343]

CoutLect, ArchLectD 45[55]; 1294 [cop. ca. 1400] ACoutBordB 410; [13 s.; cop.]

CoutMeilhanB, ArchGir 25,142[24].

FEW MINARE $6^{2}, 100 \mathrm{~b}$ : apr. menar v.a. «conduire qn à un certain endroit, vers qn ou qch» dp. $12^{\mathrm{e}} \mathrm{s}$.; LR sub mia «mener, conduire»: abéarn. miar ca. 1330 [cop. ca. 1410] HistSainte.

1 guidar (qn)

[ca. 1190] Bayonne: Establide cause es ... que neguns hom no guidi layron per lo vescomptat de Baione.

2 miar (qn)

1278 [cop. ca. 1460] Oloron: si per abenture plaze a luy que-s vulha mudar en autre senhorie, saub l'aver e-u cors segur, lo fasa-u vescoms miar entro aus termis de la senhorie.

\section{4 action de conduire:}

1. menament (de loc en loc) m. 1236 [cop. 14 ${ }^{\mathrm{e}}$ s.] PrivEDM[D], ArchGir 3,103= Lv 5,188b «Führen».

FEW MINARE $6^{2}, 103 \mathrm{a}$ : agasc. menament $\mathrm{m}$. «action de conduire» Bordeaux 1236 [= l'attestation ci-dessus].

\section{5 sauveté, lieu d'asile:}

1. «lieu franc où l'immunité de l'individu est respectée»

saubetat f. [1220; cop. ca. 1460] ForMorl v. loc de saubetat, ci-dessous 2; en la saubetat et en la villa de Memisan 1270 [cop. 1380] ChartSSeverPC 484/486 = Lv; 1278 [cop. ca. 1460] ForOlDC 127a[25] = LR sub saubetat: sauvetat (v. aussi donar saubetatz ad sub accorder une sauveté à 2676,1); en gleissa o en saubetat [13 ${ }^{\mathrm{e}}$ s.; cop. ca. 1400] ACoutBordB 26;27[7] = Lv.

FEW SALVUS 11,134a: agasc. saubetat f. «lieu d'asile»; Lv 7,453b «Freistatt»: saubetat (de Sancta-Crotz) 1414 JurBord; citat, saubetat, bastida 1372 [cop. 1378] Bordeaux, sauvatat (de Sent-Andriu) 1396 Bordeaux. 
$\diamond$ Rem. Cf. LR: «Immunité, un privilège de franchise, en ce sens que dans ces lieux (bourgs, villes, territoires) nul ne pouvait être ni attaqué, ni contraint; chacun y était sauvegardé dans sa personne et son, avoir ${ }^{`} »$. Le terme désigne aussi une bourgade jouissant d'un droit d'asile. Afin de développer des régions essentiellement désertes, des bourgades rurales neuves offrent aux populations et à leurs biens la «securitas» (p. ex. contre la violence des guerres féodales).

2. «(être en) lieu de sauveté»

(esser en) loc de saubetat synt.m. [1220; cop. ca. 1460] ForMorl, ForsB[OG] $328[32]$.

À rattacher à FEW LOCUS 5,392 et SALVUS 11,134a.

1 saubetat

1278 [cop. ca. 1460] Oloron: Sober asso establi et done saubetatz ad aqueste ciutat, en tau conbent que nulh estrani no y fase nulh embadiement ad augun homi dentz los termis de la saubetat.

2 (esser en) loc de saubetat

[1220; cop. ca. 1460] Morlaàs: que eg sie en loc de saubetat (v. sub donner saufconduit 2672,4a).

\section{6 accorder une sauveté à (une ville, un lieu):}

1. donar saubetatz ad (aqueste ciutat) loc.verb. 1278 [cop. ca. 1460] ForOlDC $127 \mathrm{a}[25]=\mathrm{LR}$.

À rattacher à FEW DONARE 3,136a et SALVUS 11,134a.

1 donar saubetatz ad

1278 [cop. ca. 1460] Oloron: done saubetatz ad aqueste ciutat (v. sub sauveté, lieu d'asile 2675,1).

b) L'homme au travail

2. L'agriculture, l'élevage, le jardinage

aa) La ferme et ses dépendances, le bétail, l'élevage

1. La ferme et le bétail

2677 paysan, agriculteur:

1. «paysan(s), exploitant(s)»

paies m.sg. et pl. [ca. 1186; cop. ca. 1250 et après 1425] CartBigRC [51,9;7: 1694 
var. $\mathrm{B} ; \mathrm{Pb}$ ]; pales [leçon à corr. en paies d'après les mss $\mathrm{B}$ et $\mathrm{Pb}$ ] [ca. 1186; cop. ca. 1390] CartBigRC 70[51: ms. Pa et n202]; [ca. 1238; cop. ca. 1390] CartBigRC 104[71,46 et n202: ms. Pa], pages 1203 [cop. 1345] CoutSGaudensM 26[18];58 [77].

FEW PAGENSIS 7,468b: apr. pagés m. «paysan» lang. Quercy, rouerg. gasc. $12^{\mathrm{e}}-14^{\mathrm{e}}$ s., Rn; etc., gasc. id. $16^{\mathrm{e}}-17^{\mathrm{e}} \mathrm{s}$., RevGasc 18,183; RF 23,303, béarn. pagès $[=\mathrm{LR}]$.

$\diamond$ Rem. Pour la forme paies v. CartBigRCGloss.

2. «cultivateur»

cuytivayre m. 1238 [vid. 1278; cop. 14 ${ }^{\mathrm{e}}$ s.] PrivEDM[D], ArchGir 3,122.

ø FEW COLERE 2,886b; Rn 2,443b/444a: apr. cultivador «cultivateur» $13^{\mathrm{e}} \mathrm{s}$. EvSMarc; $\left[14^{\mathrm{e}} \mathrm{s}.\right]$ TradPass.

3. «laboureur»

laborador m. 1238 [vid. 1278; cop. 14 ${ }^{\mathrm{e}}$ s.] PrivEDM[D], ArchGir 3,121;122; 1269 AnglGuyB 161b;206b; lauraire 1265 [cop. $17^{\mathrm{e}} \mathrm{s}$.] CoutLarrC 121[15].

FEW LABORARE 5,104a: apr. laurador «laboureur», Arrens lauradoú P 19; Lv 4,336a sub 1 «Ackersmann»: agasc. laurador 1308 CoutGers; Rn 4, 3b: laborador «laboureur» ca. 1465 Bordeaux; LR sub lauradou: abéarn. laborador «id.» $15^{\mathrm{e}} \mathrm{s}$. BaronBéarn, lauradoo ForsB 1551 (les deux aussi sub manestrau), laurador (de sons camps) «laboureur (de ses champs)» ca. 1330 [cop. ca. 1410] HistSainte, béarn. lauradou ca. 1700 FondCalv.

4. gazanher m. 1265 [cop. $14^{\mathrm{e}}$ s.] PrivMonségur, ArchGir 5,7 = Lv sub gazanhier [sans déf.].

$ø$ FEW abfrq. *WAIĐANJAN 17,461b.

1 pages

1203 [cop. 1345] SGaudens: E si lunhs pages de Comenge se-n entra en Sent Gaudens per estar deuen le les prosomes aiudar si obs 1-es.

2 cuytivayre

1238 [vid. 1278; cop. $14^{\mathrm{e}} \mathrm{s}$.] Entre-deux-Mers: Item, si no es donat au prebost aquo que demanda manda et remanda en menan en longuas partidas affinque cessian de lurs obras ... entro que satisfassa a la cupiditat de lor dels mesquinx laboradors o cuytivayres.

\section{3 laborador}

1238 [vid. 1278; cop. $14^{\mathrm{e}}$ s.] Entre-deux-Mers: lodeyt laborador bulha dar aus cavatz ... mixtura o autra competent blat, segont la costuma de la terra (v. aussi cidessus 2). 


\section{8 qui cultive, laboure la terre:}

1. laborador adj. 1270 [n.st.] CartSCrD, ArchGir 27,218[231].

ø FEW LABORARE 5,105b; LR sub lauradou: béarn. (sourdat) lauredou «(soldat) laboureur»; cf. Lv 4,336b sub laurador «zum Ackern, Pflügen dienend»: agasc. (bestials) laurados pl. 1484 Nogaro.

\section{1 laborador}

1270 [n.st.] Bordeaux: aissi cum lodeit arener se boitera, lo medis Arnaud lo deu plantar et far plantar de bona planta de bit à l'esgart de prodomes laboradors.

\section{9 fermier, métayer:}

1. borde $[\boldsymbol{r}]$ m.: bordes pl. 1251 AnglGuyB 168a; id. 1258 [cop. ca. 1290] CartFronsacTD, ArchGir 38,31; id. 1269 AnglGuyB 206b; bordés pl. 1270 [cop. 1380] ChartSSeverPC 472; bordes pl. 1284 ChartAlbrM 143[112].

FEW abfrq. BORD 151,188a: abéarn. border m. «métayer» [= LR sub bourdè: 1385 DénBéarn], béarn. bourdè.

2. «celui qui dirige une ferme, une métairie d'un monastère» granger m. [1288; cop. ca. 1300] CoutMontsaunèsM 2a.

FEW *GRANICA 4,226b: abéarn. granger $\mathrm{m}$. «celui qui a la direction d'une métairie appartenant à un monastère» [=LR: «régisseur d'une grange» [s.d.] ArchBPyr]; Rn 3,496b: grangier «granger» 1263 Doat [alang. ou agasc.?].

\section{1 borde $[r]$}

1251 Guyenne [concern. Blanquefort]: medissa Na Mabila aue ni auer deue en mediss castet de Borc, ni en la honor ... ni sobre-us cauoirs, ni sobre-[u]s bordes, hommes, habitants, ni habitadors en mediss castet.

\section{2 granger}

[1288; cop. ca. 1300] Montsaunès: Arnaut de Castelho, Marrone de Arroeda, grangers de la baylia de la dita mayso, e dels autres frayres nostres de la dycha baylia.

2680 cultiver [v. aussi travailler, labourer (la terre, le champ, la vigne) 2750; terre cultivée 2710]:

1. «cultivé (d'une terre agricole)»

coler v.tr. [colt, cout, couit, cot p.p. à valeur adj.] [1239] LAg v. terra couta; 1256 Casteljaloux v. terra colta; 1256 [cop. ca. 1320] CartBlanc v. tera colta; 1260 Bagnères-de-Bigorre v. terre coute; 1270 Lectoure v. terra co(u)ta, terra cauta; 1272 TextBig v. terre coute; 1274 [n.st.] Mill v. tere cote; ca. 1274 AnglGuy v. 
terra couta; 1277 [cop.] Mill v. terre cote; 1289 [n.st.; cop. 1667] Bordeaux v. terra coutta; 1291 ChartAlbr v. terra couta; ca. 1300 StatCaudecoste v. terra couita sub terre cultivée 2710,1 ; cult, cault p.p. 1276 [n.st.; cop. $17^{\mathrm{e}}$ s.: concern. le château de Benauge (Gir.)] v. (causa) caulta; 1294 [cop. ca. 1480] CartDax v. (h)eretat culte sub 2710,1.

FEW COLERE 2,886a/b: abéarn. coler «cultiver», cole [= LR sub cole: abéarn. colt «(terrain) cultivé» (sans indication de source)].

2. cautiuar v.tr. 1257 ChartAlbr v. terra cautiuada sub terre cultivée 2710,2, cultibar 1276 [cop. ca. 1330] CoutFez v. hono[r] cultibada sub 2710,2; coltivar [1288; cop. ca. 1300] CoutMontsaunès v. terra coltivada sub 2710,2, coitivar 1282 [cop. 1356] MartOrth v. terre coitivade sub 2710,2, coitiuar 1300 Mill v. terre coitiuade sub 2710,2, coytiuar 1293 [n.st.] ChartAlbr v. terra coytiuada sub 2710,2 .

FEW COLERE 2,886b: abéarn. coytibar «cultiver (la terre)» avec n1, béarn. couytibá «cultiver la terre», coeytibá, Arrens coutibá; ib. 887a: abéarn. cultibar «cultiver (la terre, une plante)», béarn. cultibá; LR sub coytibar: abéarn. (terres) coytivades e a coytibar «(terres) cultivées et à cultiver» [s.d.] ArchBPyr, ib. sub cultiba; exartiga: cultibar «cultiver (pièce de terre)» [s.d.] ArchBPyr.

3. acoitivar v.tr. 1246 [cop. $15^{\mathrm{e}} \mathrm{s}$.] CartSSeurin v. terra acoitivada sub terre cultivée 2710,3 , acoutivar 1256 [cop. $15^{\mathrm{e}} \mathrm{s}$.] CartSSeurin v. terra acoutivada sub 2710,3 .

FEW COLERE 2,886b: apr. acoltivar «cultiver» LvP, gasc. acoueitiouá Ad 68, Gers «cultiver avec soin», cf. ib.: Lavedan acoutibat «terre cultivée avoisinant les terrains incultes».

4. gazanhar v.tr. 1252 [n.st.; cop. $15^{\mathrm{e}} \mathrm{s}$.] CartSSeurin v. terra gazanhada sub terre cultivée 2710,5, gadanhar 1258 CartSCr v. terra gadanhada sub 2710,5, gaaniar, gaiar 1258 [cop. ca. 1290] CartFronsacTD, ArchGir 38,31.

ø FEW abfrq. *WAIĐANJAN 17,461a/b: apr. gaagnar v.a. «cultiver» ClermF. 1195, Brunel, gaainar ibid., gazanar rouerg. 1155; Moissac 1197; Toulouse 1200, gaanhar Limoges 1256, Toulouse gazanhá «labourer».

4 gaaniar

1258 [cop. ca. 1290] Fronsac: aqui apres va XII sazons, e las oit van e la mar en dosoa au boscs, tant cum hom ne pot gaaniar.

\section{1 ferme, métairie:}

1. «exploitation agricole, spécialet. son centre ou ses bâtiments d'exploitation, ferme, métairie»

borde f. 1250 [cop. ca. 1460] ForAspe, ForsB[OG] 538[10;11: éd. trad. «borde», 
gloss «grange»]; 1260 Bagnères-de-Bigorre, RecGascL 37[21]; [1300] CoutAure, Monl 6,55;56, borda [ca. 1250; cop. ca. 1390] CartBigRC 9[2,11];11[2,30;36]; 1179 Montsaunès, RecGascL 5[4]; [ca. 1280; cop. 1343] EtLect, ArchLectD 60 [16];65[39]; 1294 [cop. 1343] CoutLect, ArchLectD 51[85]; 1295 [cop. ca. 1330] CoutFezB, ArchGir 27,388[30].

ø FEW abfrq. BORD $15^{1}, 187 \mathrm{~b}$ : apr. borda $\mathrm{f}$. «métairie, habitation agricole» Rn; Lv[P]; etc., gasc. bordo «métairie» ALF 1851, béarn. borde «cabane»; LR sub borde: abéarn. borde «ferme, métairie» [sans indication de source], ib. sub affiusa: $15^{\mathrm{e}} \mathrm{s}$. BaronBéarn; Palay sub borde: «désigne parfois la ferme, la métairie, parfois seulement les bâtiments autres que le bâtiment servant à l'habitation des personnes».

$\diamond$ Rem. La dualité sémantique de borde «bâtiment annexe séparé de l'exploitation principale (grange)» et «exploitation agricole considérée dans son ensemble/unité d'exploitation (ferme, métairie)» est difficile à distinguer dans nos textes. CartBigRCGloss sub borda se réfère à Cursente 1998 (v. ci-dessous sub 7), p. 19: «1. jusqu'au XIII ${ }^{\mathrm{e}}$ siècle le centre d'une exploitation agricole de statut indéterminé; 2. à la fin du Moyen Âge, dans le secteur pyrénéen, l'annexe de la maison destinée à abriter le fourrage et le bétail; 3. à la fin du Moyen Âge, dans le restant de la Gascogne, l'unité principale d'habitation et l'exploitation isolée dans le terroir, par rapport à l'hospitium du bourg». V. aussi X. Ravier, «Lexique du peuplement», dans Nouvelle revue d'onomastique 1998, $\mathrm{n}^{\text {os }} 31-32,50-52$.

2. «domaine d'une borde, centre d'une exploitation agricole, ferme»

bordiu m. 1246 [cop. $15^{\mathrm{e}}$ s.] CartSSeurinB 157; 1253 [n.st.] CartSCrD, ArchGir 27,159; 1256 [cop. $15^{\mathrm{e}}$ s.] CartSSeurinB 233; 1257 ChartAlbrM 217; 1263 CartSCrD, ArchGir 27,228; 1273 [cop. 15 s.] CartSSeurinB 339[344]; 1278 [n.st.] Bordeaux, ArchGir 43,173; 1283 ChartAlbrM 128[105]; [13 $\mathrm{e}$.; cop. ca. 1400] ACoutBordB 31[16: tala de bordius];174[227], bordil [13 ${ }^{\mathrm{e}}$ s.; cop.] CoutMeilhanB, ArchGir 25,144[37].

FEW abfrq. BORD $15^{1}, 187 \mathrm{~b}$ : apr. bordil m. «métairie» $14^{\mathrm{e}} \mathrm{s}$., gasc. bourdilh «petite ferme», Gers bourdiou «habitation du métayer», béarn. bourdiu; Rn 2,238a: bordil «métairie, ferme» $14^{\mathrm{e}} \mathrm{s}$. Doat [alang. ou agasc.?]; LR sub bourdiu: abéarn. bordiu «maison de ferme, ferme» $\left[14^{\mathrm{e}} \mathrm{s}\right.$.] EnqBéarn.

3. «petite ferme (ou ensemble de bâtiments agricoles) ?»

bordel m. 1161 [cop. ca. 1290; cont. lat.] CartGimC 325[39].

$ø$ FEW abfrq. BORD $15^{1}, 188$ a.

$\diamond$ Rem. Acception difficile à discerner: «petite ferme» ou «ensemble de bordes» (?) (suffixe soit diminutif, soit collectif); cf. DC 1,706b: mlt. bordelum «tuguriolum» 1209 et Palay: bourdèu «ensemble de bordes [bâtiments agricoles ?]». 
4. «domaine d'une exploitation agricole, ferme»

bordaria f. [990; cop. $17^{\mathrm{e}} \mathrm{s}$. (cont. lat.)] CartRéole, ArchGir 5,108.

FEW abfrq. BORD $15^{1}, 188 \mathrm{a}$ : apr. bordaria f. «métairie» dp. $12^{\mathrm{e}} \mathrm{s}$.; Rn 2, 238a: id. «borderie, petite métairie, petite maison de campagne»1194; 1231 Doat [alang. ou agasc.?].

5. «ferme, domaine rural»

mayne $\mathrm{m} .1275$ AnglGuyB 238a; 1291 ChartAlbrM 72;243; [13e s.; cop. ca. 1400] ACoutBordB 84[95: éd. trad. «domaine principal»] = Lv, maine 1291 ChartAlbrM 240.

FEW DOMINIUM 3,130b: Teste maine «terre cultivée»; Lv 5,22b sub maine: agasc. mayne [sans déf., mais avec renvoi à $\mathrm{M}$ : maine «domaine, terre cultivée, en Guienne» et LR: béarn. mayne «ferme, domaine»]; 1414 JurBord; 1475 SFoy-la-Grande, ArchGir 1,181; 1338 SLaurent-Médoc, ArchGir 6,185; 1305 et 1322 Vayres, ArchGir 6,214;8,94; 1460 Bordeaux, ArchGir 10,127, ib. sub mainamen: 1426 [n.st.] Bordeaux, ArchGir 1,36.

6. «exploitation agricole, ferme, propriété (destinée à l'élevage?)»

boaria f. [ca. 1085; cop. ca. 1390 (cont. lat.)] CartBigRC 15[3,28: ms. Pa, gloss: «bouverie, ferme spécialisée dans l'élevage des bœufs, étable à bœufs»]; [ca. 1165; cop. ca. 1390] CartBigRC 7[1,37: ms. Pa]; 1288 [cop. ca 1300] CoutMontsaunèsM 57a[55: trad. «métairie»], boarie ca. 1180 [cop. après 1425] CartBigRC [19,18: var. Pb]; 1296 [cop. 1406] LVertBénacB 205[11]; s.d. [13 s.; cop. 1406] LVertBénacB 187[23], boerie $12^{\mathrm{e}} \mathrm{s}$. [cont. lat.] CartSordeR 139[162]; 1281 [cop. 14 s.] DocSPéB 395, boeria [ca. 1165; cop. ca. 1250] CartBigRC [1,51: var. B]; [ca. 1180; cop. ca. 1250] CensIbos, CartBigRC [19,20: var. B]; boria 1235 Bordeaux, RecGascL 118[54]; 1247 [cop. 15 s.] CartSSeurinB 194;195; 1263 CartSCrD, ArchGir 27,225; 1279 [n.st.] Bordeaux, ArchGir 43,201; 1283 ChartAlbrM 120; 1291 ChartAlbrM 71.

FEW mlt. BOVARIA 1,476a: apr. bo(a)ria «métairie»; LR sub boarie: abéarn. boaria «id.» ForsB 1551, boeria $15^{\mathrm{e}}$ s. BaronBéarn, ib. sub borde; poble: boria, borie $15^{\mathrm{e}} \mathrm{s}$. BaronBéarn.

7. «domaine rural/habitation rurale, manoir entouré de terres (ensemble de la tenure et des droits commandés par une maison)»

casal m. ca. 1125 [cop. 14 ${ }^{\mathrm{e}}$ s.; cont. lat.] CartSordeR 121[145]; 1143 [cop. 1249] CartLézat, RecGascL 5[3]; [ca. 1160] Comminges, Brunel 94[97; gloss „habitation rurale"]; [1184] Comminges, Brunel 198[210]; 1187 Comminges, BrunelS 122 [488, gloss «habitation»]; [ca. 1190; cop. 13 ${ }^{\mathrm{e}}$ s.] Bouzin, ActesBonnefontSH 259 [576]; Brunel 343[348,3: ca. 1200, date à corr., cf. Dinguirard, Lengas 12,57 n16]; $12^{\mathrm{e}} \mathrm{s}$. [cop. 1249] CartLézat, RecGascL 4[3]; [12 ${ }^{\mathrm{e}}$ s.; cop. ca. 1390] CartBigRC 15 [4,4;8], casau ca. 1125 [cop. 14 s. s.; cont. lat.] CartSordeR 121[145]; [ca. 1190] ChMalf, Bal 1,422; ca. 1190 [cop. $18^{\mathrm{e}}$ s.; cont. lat.] ActesBonnefontSH 258[573]; $12^{\mathrm{e}} \mathrm{s}$. [cop. $14^{\mathrm{e}} \mathrm{s}$.] CartSordeR 139[162] = LR sub que 1: «domaine» ca. 1200; 
RecGascL 74; [ca. 1238; cop. ca. 1390] CartBigRC 104[71,45];105[69]; 1259 Mill, ArchGir 45,2[1,5]; [av. 1272; cop. 1406] LVertBénacB 178[25]; 1277 [cop.] Mill, ArchGir 45,67[20].

FEW CASALIS 2,454a: abéarn. casal «métairie, manoir entouré de terres»; LvP: aoccit. cazal «maison»; LR: «anciennement, casau, casal, domaine rural; maison et terres qui en dépendaient».

$\diamond$ Rem.: Pour la définition de ce vocable dans la Gascogne médiévale v. B. Cursente, Des maisons et des hommes. La Gascogne médiévale ( $\mathrm{XI}^{\mathrm{e}}$ $\mathrm{XV}^{\mathrm{e}}$ siècle), Toulouse 1998. Aujourd'hui, en gascon, ce mot ne signifie plus que «jardin» [v. sub jardin 2846,3].

8. cazalera f. [1288; cop. ca. 1300] CoutMontsaunèsM 22a[17: trad. «ferme»].

ø FEW CASALIS 2,454b: cf. apr. cazaliera «terrain à bâtir»; Rn 2,348b: casalera $\mathrm{f}$. «cassine» $13^{\mathrm{e}} \mathrm{s}$.

9. «petite ferme»

casalhon m. 1247 [cop. $15^{\mathrm{e}} \mathrm{s}$.] CartSSeurinB 213.

$\varnothing$ FEW CASALIS 2,454b: cf. Teste cazailloun «joli petit jardin»; cf. M: gasc. casalhoun «petite masure, cahute en pierres sèches; petit jardin».

10. «métairie (dépendant d'une seigneurie ?)»

tenguda f. 1243 AnglGuyB 167b; RecFéodD, ArchGir 3,3[3] = Lv 8,164b sub 3 «Pachtgut, Meierhof» [ArchGir 11 Gloss «tènement»].

FEW TENERE $13^{1}, 220$ b: apr. tenguda f. «possession d'un bien» $12^{\mathrm{e}} \mathrm{s}$., Lv; BrunelS; «bail, fermage» 1490, Pans 5; «territoire» gasc. $13^{\mathrm{e}}-14^{\mathrm{e}} \mathrm{s}$.

1 borda

1295 [cop. ca. 1330] Fezensaguet: bordas, hostals ne autres locz poblatz (borde v. sub étable 2687,3).

2 bordiu

1246 [cop. $15^{\mathrm{e}} \mathrm{s}$.] Bordeaux: E es asaber ... que-u camins qui entra en la palu qui es davant lo bordiu en P. de Lescapon es ancians camins comunaus.

3 bordel

1161 [cop. ca. 1290] Gimont [cont. lat.]: vendidit terram dez Solan que est subtus pred. ecclesiam et unum conturnum de terra as Albacoa et alterum conturnum subtus poi de da bordel.

4 bordaria

[990; cop. $17^{\mathrm{e}} \mathrm{s}$.] LaRéole [cont. lat.]: Frater Guillelmus de Causago fecit donum Sancto-Petro de IIII dinaradas de vinea et una bordaria.

5 mayne

1275 Guyenne: e Andreu de Carbonera deu ... sobre-u mayne e demindanuda (sic.) .j. quarta de siuada. 


\section{6 boaria}

[ca. 1085; cop. ca. 1390] Lavedan [cont. lat.] : De boaria de Sant Criac ostem.

7 casal, casau

ca. 1125 [cop. $14^{\mathrm{e}} \mathrm{s}$.] Sorde [cont. lat.]: Terra similiter que est suptus ecclesiam, inter lo casal de Camiade et inter lo casau de Bainas. - 1259 MMarsan: lo casau de Cab-de-Biele, e ab totes les terres e ab totes les pertiences qui au dit casau apertienen.

8 cazalera

[1288; cop. ca. 1300] Montsaunès: De las maysons. Item, volem qu e-ls devant ditz homes e lors successors per tostemps aian e tengan lors mayos e cazaleras en aysi cum ara an e tenent.

9 casalhon

1247 [cop. $15^{\mathrm{e}} \mathrm{s}$.] SSeurin: la via travensana qui es plus pres desus endosca aus casalhons qui foren Arn. Johan ...

10 tenguda

1243 Guyenne: bougo que lur [Lv corr.: lus] auandit homme e lur her, e aquit qui tiendran las tengudas que li mediss home tenen, arredan et paguen a l'auandit N'Arrams ... cada an, a la Santa Maria de miehs Aost.

\section{2 tenancier, maître d'un casal:}

1. casaler m. [ca. 1250] ActesBonnefontSH 263[586].

FEW CASALIS 2,454a: abéarn. casaler m. «paysan; roturier», agasc. «tenancier d'un casal» Comminges 1200 [date à corr., v. l'attestation ci-dessus], Brunel Ch; LR sub casalèe: abéarn. casaler «anciennement, celui qui occupait, exploitait un casau», «roturier, vilain» [s.d.] ArchBPyr.

1 casaler

[ca. 1250] Bonnefont: en P. que dona an Fortanér .C. solz per que azo lo fes e-1 e sos casalers en Arramon W. XII. dines de sens.

\section{3 maison d'habitation d'une ferme:}

1. «habitation d'un domaine rural»

mas m. [ca. 1185] Comminges, Brunel 220[227,9]; 1187 Comminges, BrunelS 123[488,7]; 1249 [n.st.; cop. ca. 1290] CartFronsacTD, ArchGir 38,4.

FEW MANSUS $6^{1}$,261b/262a: apr. mas «habitation rurale, ferme» ca. 1109$16^{\mathrm{e}} \mathrm{s}$., Rn; Brunel(S); etc.; gasc. $i d$. «grande maison de campagne» D, Gers $i d$., béarn. $i d$. (, vieux'). 
2. «maison-chef du mas, manoir principal d'un domaine»

a) campmas m. 1270 Lectoure, DocGalardN 4131;34, capmas 1270 Lectoure, DocGalardN 4 ${ }^{1}, 34$.

FEW CAPUT 2,343a: apr. capmas «habitation principale d'un domaine» $12^{\mathrm{e}}-13^{\mathrm{e}}$ s., rouerg. Quercy, Rn; Brunel[Gloss: cabmas, capmas «chef-mas, habitation principale d'un domaine»], camas Aude $16^{\mathrm{e}} \mathrm{s}$. et MANSUS $6^{1}$, 264b: apr. capmas m. «manoir principal d'un domaine»; Rn 4,148a: cammas «campmas, habitation principale» 1266 Doat [alang. ou agasc.?].

b) cap del mas synt.m. 1125 [cop. ca. 1390] CartBigRC 64[64,71: «maison principale du manse»].

À rattacher à FEW CAPUT 2,343b et MANSUS $6^{1}, 264 \mathrm{~b}$.

1 mas

[ca. 1185] Comminges: Aquest do [fo] feit deuant lo portal del mas de Sent Gaudenz.

2a campmas

1270 Lectoure: castel, o vilas, o campmas.

$2 \mathrm{~b}$ cap del mas

1125 [cop. ca. 1390] Bigorre: Aquest pleit fo feit e-1 monestier dauant lo cap del mas de Sent Auenti.

\section{4 grange:}

1. borde f. [1220; cop. ca. 1460] ForMorl, ForsB[OG] 334[41, gloss «grange»]; [1288; cop. ca. 1460] ForGén, ForsB[OG] 292[263].

$\varnothing$ FEW abfrq. BORD $15^{1}$,187b: apr. borda f. «grange» Villeneuve-lès-Lavau 1489, Castillon bordo; LR sub borde;poble: abéarn. borda «grange» $15^{\mathrm{e}}$ s. BaronBéarn, ib. sub troulh: ForsB 1551; sub artigau: borde 1520 CoutSoule; sub assoula: [s.d.] ArchMontaut; sub toumbá: [s.d.] ArchBPyr. $\diamond$ Rem. V. le comment. sub ferme, métairie 2681,1.

1 borde

[1220; cop. ca. 1460] Morlaàs: Si per abenture augun deus borzees . . . arde sas maysons o borde o molii.

\section{5 hangar, appentis:}

1. «construction légère en bois (pas nécessairement appuyée à un mur)» apentis f. [1262; cop. ca. 1400] Pad, ACoutBordB 193.

FEW APPENDERE 25,33a: aland. appentix $\mathrm{m}$. «petit toit à une seule pente, 1702 
appuyé à un mur, tandis que la partie inférieure est soutenue par des poteaux»; cf. aussi ib.: abéarn. apendis «dépendances» [LR: LOr = apendes ca. 1205 [cop. $15^{\mathrm{e}} \mathrm{s}$.] CartOssauT 42 [à traiter sub appartenances, dépendances, B III $\left.b_{6}\right]$.

2. apent m. 1278 [n.st.] Bordeaux, ArchGir 43,173.

FEW APPENDERE 25,33a: Gers apen «appentis», Teste appan, gasc. apent «bergerie».

\section{1 apentis}

[1262; cop. ca. 1400] Bordeaux: Item, didem que la plassa de Sent-Andriu es paduent, et l'apentis qui es aqui en loc public deu ne estre ostada.

2 apent

1278 [n.st.] Bordeaux: en B.B. . . . a dat . . . a H.C. . . totz los mantz, e dreitz . . . de tot aquet son bint de terra, ab l'apent qui es, ab totz sos apartenementz, qui es au loc aperat a Las Soys.

\section{6 pailler, endroit où est entreposée la paille:}

1. «grange, grenier ou hangar de paille» paltrer [1. palher ?] m. ca. 1300 StatCaudecosteB, ArchGir 31,235[66].

FEW PALEARE 7,502b: cf. apr. pailler $\mathrm{m}$. «grenier à paille» rouerg., Brunel; cf. Rn 4,401: apr. palhier «grenier à paille» ca. 1270 GuirRiq.

\section{1 paltrer}

ca. 1300 Caudecoste: E metem mays en nostres establimens que tout auc o tota auca que sia trobat o trobada . . en cazal o en paltrer ... que-s guatge II d. arn. si la mala facha era paruent.

\section{7 étable:}

1. «écurie»

estrabla f. [13 $\mathrm{e}$ s.; cop. ca. 1400] ACoutBordB 57[53]; estable m. [13 ${ }^{\mathrm{e}} \mathrm{s}$; cop. $15^{\mathrm{e}} \mathrm{s}$.] ACoutBordB 57[53: var.].

FEW STABULUM 12,222b/223a: apr. estable m. «écurie, lieu où l'on loge les chevaux»; gasc. establo f. «étable» M, béarn. estable; Lv 3,288b: agasc. establa f. «Stall, Stallung [à corr. écurie]» 1483 ArchLect; stapla 1485 CConsRiscle; LR: abéarn. estable «écurie» ca. 1377 RôlesGPhébus.

2. «étable à bœufs»

boarenca f. 1276 [cop. ca. 1330] CoutFezB, ArchGir 27,360[27].

ø FEW mlt. BOVARIA 1,476a. 
3. «bergerie»

parc $\mathrm{m}$. [1300] CoutAure, Monl 6,55.

FEW PARRICUS 7,663b/664a: apr. parc $\mathrm{m}$. «clôture légère et transportable où l'on enferme les moutons quand ils couchent dans les champs» lang. rouerg. $12^{\mathrm{e}}-15^{\mathrm{e}} \mathrm{s}$., Rn; Lv, etc., béarn. Labouh. «bergerie»; LR: abéarn. id. «parc, bergerie» [sans indication de source].

1 estrabla

[13 ${ }^{\mathrm{e}}$ s.; cop. ca. 1400] Bordeaux: si un cabat aucy un home en l'estrabla.

2 boarenca

1276 [cop. ca. 1330] Fezensaguet: si algun de lor aura tengut o tenga sa companha o familia brasses o logatz o autres, fora la dita bila, en cabana o en cabanas, boarenca o boarencas,... .

3 parc

[1300] Aure: Item si en lad. val d'Aure nul . . . met foc en borde ou en maison, ou en autre parc ... incontinent se prengue et mette en prison.

bétail: v. bétail 1237; gros bétail 1238; menu bétail 1287

sabot: v. 1214

\section{8 qui tète (jeune animal):}

1. popant adj. [1300; cop. 1466] CoutCastBouz, Monl 6,98 [add. à petit d'un animal 1232].

ø FEW *PUPPA 9,605b: apr. popar v.a.n. «téter» $14^{\mathrm{e}}$ s. [= Elucidari, Rn 4, 600b «remâcher», corr. par Lv 6,458a: animaus popans «(an der Mutterbrust) saugen»].

1 popant

[1300; cop. 1466] Castéra-Bouzet: et en tota autra bestia caualina .IV. dinés Arnaudencs si no es popant, et en mul o mula si no es popant .IV. dines Arnaudencs.

\section{9 nourrir, élever (un animal):}

1. «donner à manger (aux bêtes, au bétail)»

dar a minjar a (un animal) loc.verb. 1296 [cop. 1336] EtBay 254.

À rattacher à FEW DARE 3,14b et MANDUCARE $6^{1}, 162$.

2. «spécialt., engraisser (en parl. d'un cochon)»

noirir v.tr. 1203 [cop. 1345] CoutSGaudensM 56[71], nurir [1255; cop. 16 ${ }^{\mathrm{e}} \mathrm{s}$.]

CoutLaRéoleG, ArchGir 2,260. 
ø FEW NUTRIRE 7,252a; LR sub pastenc: abéarn. neurir (lors bestiars) «nourrir» [s.d.] ArchMontaut, ib. sub pastoureya: id. [s.d.] ArchBPyr.

1 dar a minjar a

1296 [cop. 1336] Bayonne: per arrezon de fulha nauere que uolossen ni ahossen obs a dar a minjar a lor boeus ni $a$ lor autre bestiar de lor parropie.

2 noirir

1203 [cop. 1345] SGaudens: Los mazeres . . . no deuen fer alhou mazed . si donx porc salat o troia no era que ed s-agues noirid.

\section{0 nourriture du bétail:}

1. «(besoins en) nourriture»

[ops] de boque et de dent loc.adv. [1288; cop. ca. 1460] ForGén, ForsB[OG] 292[263] = LR sub dalh.

À rattacher à FEW BUCCA $1,581 \mathrm{~b} / 584 \mathrm{~b}$ et DENS 3,40b.

\section{1 celui qui donne de l'avoine aux chevaux:}

1. aciuazedor m. 1265 Mill, ArchGir 45,4[9].

$ø$ FEW CIBARE 2,660a; v. DOM 93b sub [acivadador] et comment.

1 aciuazedor

1265 MMarsan: E meis que-us n-a donad e quitad totz aquedz deues que le dite madone na Mathe aue en 1-afar d-Ossan ... so es assaber: palhe e falhe e siuaze e menyar aus aciuazedors.

2692 dévorer [add. à 1220]:

1. myniar v.tr. $\left[13^{\mathrm{e}}\right.$ s.; cop. ca. 1400$]$ ACoutBordB 57.

$ø$ FEW MANDUCARE $6^{1}, 160 \mathrm{~b}$ etc.

1 myniar

[13 s.; cop. ca. 1400] Bordeaux: un porc aucy un enfant o lo mynia la man.

\section{3 hiverner (du bétail):}

1. «envoyer aux pâturages d'hiver, nourrir pendant l'hiver» exibernar v.tr. [1264; cop. 1600] PrivGavarnie, TextBigM 95[14], exsibernar [1264; cop. $16^{\mathrm{e}} \mathrm{s}$.] PrivGavarnie 95[14: var.], eisivernar, eissivernar $1268 \mathrm{Ga}-$ barret, RecGascL 87, gloss: eis(s)ivernad p.p. «qui a passé l'hiver (bétail)»= Lv. 
FEW HIBERNUS 4,421a: apr. eisivernar v.a. «nourrir (du bétail) pendant l'hiver» gasc. avec n11, Ferrère eschiuarná «hiverner (les troupeaux)», Caut. eschiberná, Lavedan chiberná, béarn. eschiberná; Lv 2,344b/345a sub eisivernar «während des Winters ernähren»: agasc. eissivernat, yssivernat adj. «der den Winter durchlebt hat» 1304 CoutAstafort, yssiuernat 1352 CoutEauze, CoutGers; LR sub exhiberna: abéarn. eschiberna «hiverner, transhumer, se dit des troupeaux qui sont conduits habituellement d'une région dans une autre pour y pâturer» [source non indiquée], béarn. eschivernar 1676 PrivRègl.

1 exibernar

[1264; cop. 1600] Gavarnie: . . . autrey de exibernar mile cinq cens caps de oelhes.

2694 fumier animal [v. aussi les excréments 1233,2]:

1. femns m. 1270 [cop. 1287] CoutSauve, CoutGersB 186[14], fems [ca. 1280; cop. 1343] EtLect, ArchLectD 67[53].

FEW FIMUS 3,544b: Bagnères hyéms «fumier», Bigorre hiéns, hiém, chém, Teste héms, béarn. Arrens hems [= LR «fumier, engrais»]; Lv 3, 431a sub fem: agasc. fems «fumier» 1314 CoutCondom.

1 femns

1270 [cop. 1287] LaSauvetat: Item, que legessa ne femns tendra deuant son ostau en la carrera, porque sia mandat e defendut (fems v. sub fosse à fumier 2695,1).

\section{5 fosse à fumier:}

1. «emplacement d'un tas de fumier ou d'ordures, dépôt, décharges publiques» femorer cominal synt.m. [ca. 1280; cop. 1343] EtLect, ArchLectD 67[53].

À rattacher à FEW COMMUNIS 2,962a et FIMUS 3,546a/b: abéarn. femerer "fumier», agasc. hemeré 1567, RF 23,301; Lv 3,434b: abéarn. fumerer «Misthaufen, -grube» 1385 DénBéarn; LR sub hemè: gasc. hemèe «fumier» 1583 Salette.

1 femorer cominal

[ca. 1280; cop. 1343] Lectoure: Totz hom e tota molher qui gitte fems, sino e-ls femorers cominals, peche .XII. dines morlas.

\section{6 amender le sol (en parl. du bétail):}

1. «laisser de la fumure (en parl. du bétail)»

femar v.intr. 1281 [cop. 14 s.] DocSPéB 396; 1294 ArbitrTarbesR 10b[11;12].

$ø$ FEW FIMUS 3,545a/b. 
$\diamond$ Rem. LR: «Dans la vallée d'Ossau, il était usage que, de la Saint-Michel de septembre à la Saint-André, chacun laissât paître et gîter dans ses terres, pour les fumer, des troupeaux de bêtes ovines appartenant à autrui, bestiar aulhii estranger per femar sas terres [contexte similaire à notre exemple ci-dessous]».

\section{1 femar}

1281 [cop. $14^{\mathrm{e}}$ s.] Pau: Eixament e-ls diitz abat e monges e la vesiau de Geyres no y devin mete vestiar estrani si donques no a fasen per femar (v. aussi sub tomber malade (animal) 2701,1).

\section{7 fumer la terre:}

1. femar v.tr. 1283 [n.st.] CartSCrD, ArchGir 27,222[239].

FEW FIMUS 3,545a/b: apr. abéarn. femar [<FIMARE] «amender (une terre) en y mettant du fumier», Dax hema 1734, RLR 31,30, BagnèresB. hiemá, Caut. béarn. hema; Rn 3,302a: agasc. terra nofemada p.p. «terre non fumée» 1314 CoutCondom; LR sub hema; (hemasou): abéarn.femar sas terras «fumer les champs» [s.d.] ArchBescat. Cf. aussi femar ca. 1390 [cop. ca. 1460] JugesMorl, ForsB[OG] 474[286].

2. femejar v.tr.: femeiar ca. 1159 [cop. $14^{\mathrm{e}}$ s.; cont. lat.] CartSordeR 116[142 n3: «répandre le fumier sur les terres [avec des bœufs]»]; id. ca. $12^{\mathrm{e}} \mathrm{s}$. [cop. $14^{\mathrm{e}} \mathrm{s}$.; cont. lat.] CartSordeR 138/139[161;162]; RecGascL 73-75 = Lv 3,431b sub femejar «düngen»; LR sub casau «charrier les fumiers» [où femeiar est précédé de carreiar].

FEW FIMUS 3,545b/546a: abéarn. femeyar [<FIMIDIARE] «fumer (la terre)» [= LR sub hemeya: femeiar ca. 1390 [cop. $15^{\mathrm{e}} \mathrm{s}$.] JugesMorl, ForsB[OG] 474(286 [var.] femeyar)], béarn. hemeya.

3. afemejar v.tr.: afemeiar $12^{\mathrm{e}} \mathrm{s}$. [cop. $14^{\mathrm{e}} \mathrm{s}$.; cont. lat.] CartSordeR 138[162]; RecGascL 73 = LR sub afemeyar renvoit à hemeya: afemeiar.

FEW FIMUS 3,546a: abéarn. afemeyar «fumer (la terre)» [< LR [graphie normalisée] à corr., v. ci-dessus]; DOM 237a.

1 femar

1283 [n.st.] Bordeaux: an promes las medissas binhas . . femar à cap de III. ans.

2 femejar

ca. 1159 [cop. $14^{\mathrm{e}} \mathrm{s}$.] Sorde [cont. lat.]: femeiar cum bobus.

\section{8 fumure:}

1. femada f. [ca. 1238; cop. ca. 1390] CartBigRC 103[71,28-29],femade [ca. 1238; 
cop. ca. 1390] CartBigRC 103[71,30;31];104[71,63];105[71,70: far f. ab buo avec n328].

FEW FIMUS 3,545b: apr. femada «action de fumer (une terre)» Lv[P]; BrunelCh, béarn. hemade «. . .; action de fumer les champs» [=LR].

$\diamond$ Rem. Far femade ab buo autrement dit, pour la corvée de fumure le censitaire doit le service de son bœuf ou de l'un de ses bœufs; v. aussi cidessous sub 3.

2. femadura f. [ca. 1175; cop. ca. 1390] CartBigRC 28[12,5: ms. Pa fermadura à corr. en femadura, conformément à la leçon des deux autres mss].

FEW FIMUS 3,545b: apr. femadura «action de fumer (la terre)» [= LvP; Rn 3,301b: alang. «engrais» ca. 1350 Elucidari].

$\diamond$ Rem. V. ci-dessous sub 3.

3. femalla $\mathrm{f}$. $\left[12^{\mathrm{e}}\right.$ s.; cop. ca. 1390] CartBigRC 18[5,31].

$ø$ FEW FIMUS 3,545b.

$\diamond$ Rem. CartBigRCGloss sub femalla: «Obligation était faite aux redevables de faire paître leurs troupeaux sur les terres du bénéficiaire de ce droit [de pâture] pendant un certain nombre de nuits».

1 femada,femade

[ca. 1238; cop. ca. 1390] Vallée d'Azun: Abbadia. II. d. e mealha e ga[ria] e anhet e porii e femada ... A Leret a .V. casaus que deuen dar ciuada e palha e ga[ria] e anhet e femade que deuen far ab buo [source: abbuo] sengles dies.

2 femadura

[ca. 1175; cop. ca. 1390] Bigorre: Lo casal de cens .V. s., [prese]nse, fermadura [1. femadura].

3 femalla

[12 ${ }^{\mathrm{e}}$ s.; cop. ca. 1390] Vallée d'Azun: Lo beguer d'Aras deu auer del cens d'Azun baca de .VI. s., . . . XL. concas de vino, femalla de uno casale.

cheval: v. bête, espèce chevaline 1254; cheval 1255; palefroi 1259; cheval de bât 1261; monture 1253; jument 1265; poulain 1267 ; pouliche 1268 ; bête de somme 1273

âne: v. âne 1275; ânesse 1276; ânon 1277; petite ânesse 1278; bête mulassière 1280; mulet 1281; mule 1282; bête de somme 1273

bœuf: v. bovin 1239; bœuf 1243; taureau 1241; vache 1245; veau 1247; taurillon, bouvillon 1248; robe des bovins 1250

mouton: v. troupeau d'ovins 1315; mouton 1317; bélier 1320; brebis 1323; agneau 1327; ovin non tondu 1337

1708 
chèvre: v. bouc 1302; bouc châtré 1304; chèvre 1306; chevreau 1308

porc: v. porc 1290; verrat 1292; truie 1293; jeune porc 1295

châtrer: v. animal châtré 1313; bouc châtré 1304

Les défauts d'une bête

2699 maigre, épuisé (d'un bovin):

1. littéralt. «(os) vidé de sa moelle», au fig. «maigre (d'un bœuf, d'une vache, par excès de travail ou par vieillesse) (?)»

desmesorat adj. [ca. 1280; cop. 1343] EtLect, ArchLectD 71[76] = Lv «ohne Mark».

ø FEW MEDULLA 6 ${ }^{1}, 636 \mathrm{~b}$ : cf. yèr. démoellé «maigre»; Lv 2,150a sub desmezolat (?) renvoit à $\mathrm{M}$ [desmesoula etc. «ôter la moelle»] avec la remarque: ,Ich weiss nicht, ob meine Annahme richtig ist; aber was könnte desmesorar sonst bedeuten?'. Mais cf. Lv 5,124a sub marfanhar: carns . . . de vilhs beus o de vaques que no aian mesoc [1. mezot, corr. ib. 275b sub mezol «Mark»] 1396 EtMarm.

Les maladies du gros et du menu bétail

2700 malade (animal):

1. malau adj. [ca. 1205; cop. ca. 1390] CartBigRC 119[71,12: adj. ou m.]; 1294 ArbitrTarbesR 9b[5;8];10b[13] (v. aussi bader malau sub tomber malade (animal) 2701,1).

$ø$ FEW MALE HABITUS $6^{1}$,89a; LR sub morboos: abéarn. malaude «(bête) malade» [ca. 1400] ForsB, ib. sub amourrou: (moton) malau [s.d.] ArchBPyr. Cf. aussi (bestiar) malau ca. 1390 JugesMorl, ForsB[OG] 428[185].

1 malau

1294 Tarbes: de bestiar saa ni malau (v. aussi sub sain (animal) 2703,1).

\section{1 tomber malade (animal):}

1. bader malau synt.verb.: si besti bat malaude f. [1273] ACoutBay, Bal 2,642 [94,2] = LR sub ja «devenir malade»; 1294 ArbitrTarbesR 10b[11;12].

À rattacher à FEW MALE HABITUS 6159a et VADERE 14,118a: abéarn. 
bade v.n. «devenir (+ adj.)» hap. $15^{\mathrm{e}}$ s., RF 23,416, béarn. baye «. . ., devenir»; Lv 5,51b: aland. bader malau (d'un homme) ca. 1468 CoutDax.

1 bader malau

1294 Tarbes: si bestiar de Tarbe y femave e-i baze malau, que daqui on femas en davant que-us des hom padoence.

\section{2 dépérir (d'une bête):}

1. se guabainhar v.pron., se gabainhar [1273] ACoutBay, Bal 2,642[94,1;2].

ø FEW *GABA 4,2a: cf. béarn. gabanhá «détériorer qch» [= LR sub gabanha: abéarn. gabanhar; se gabanhar «se détériorer» [s.d.] ArchBPyr], agasc. s'engavanhar «s'endommager».

\section{1 se guabainhar}

[1273] Bayonne: Si augun ha logat a rossin o autre besti de cabaugar, si le besti mor o-s guabainhe degudementz mian, lo loguedor no es tingut mas de loguer tant solementz pagar trou lo die que ne se-n puyra seruir.

\section{3 sain (animal):}

1. sa adj. [ca. 1205; cop. ca. 1250] CartBigRC 118[71,11], saa 1294 ArbitrTarbesR $9 \mathrm{~b}[5] ; 10 \mathrm{~b}[13]$.

FEW SANUS 11,189a: apr. san, sa adj. «sain», béarn. saa; LR sub alègre: abéarn. (bestiar) $s a[a]$ adj. «sain» [s.d.] ArchBPyr, ib. sub impedemia: (bacas) saas pl. [s.d.] ArchBPyr.

$1 s a$

[ca. 1205; cop. ca. 1250] Bigorre: E d'amas las partidas que no-s deuen encausar ni empegner los aues sas estan e aus malaus que deuen dar padoensa ( $s a a$ v. sub malade (animal) 2700,1).

\section{La cour}

\section{4 cour:}

1. «cour (de ferme)»

cor f. 1282 [cop. 1356] MartOrthB 94[9].

$ø$ FEW COHORS 2,850a; LR sub cour «basse-cour, cour destinée aux écuries, aux étables»: béarn. cour (de bestiaa) f. «cour (de bétail)».

1 cor

1282 [cop. 1356] Sauveterre: aver padoenc e espleit de lors cors o cortilhs. 
bb) Le sol

\section{5 terrain, terre:}

1. «étendue limitée de terres cultivables, considérée comme objet de possession (propriété, domaine, terrain)»

terres f.pl. 1296 [cop. $16^{\mathrm{e}} \mathrm{s}$.] Bayonne, EtR 2,81[3]. - V. aussi terra, tera, terre, tere sub pièce parcelle de terre 2706,$1 ; 2 ; 3$; terre inculte, en friche $2709,4 \mathrm{a} ; \mathrm{b} ; \mathrm{c} ; 5$; terre cultivée 2710,$1 ; 2 ; 3 ; 4 ; 5$; fixation des limites de terre 2713,1 .

ø FEW TERRA $13^{1}, 245$ a.

2. plaza f., plaze 1256 [cop. ca. 1320] CartBlanc, CartAuchL 219[3].

FEW PLATEA 9,37b: aland. plasse «terrain; champ» $15^{\mathrm{e}}$ s., land. «champ»; Lv 6,366a sub 7 «Stück Land»: alang. plassa de terra ca. 1377 Montpellier.

3. «ensemble de terrains (dépendant d'un lieu-dit ou d'une maison), terrain, territoire»

a) terrador m. 1255 [cop. 1341] CoutMontrOG 140[43]; [1288; cop. ca. 1300] CoutMontsaunèsM 7a[5: trad. «terroir»];21a[16: «id.»].

FEW TERRITORIUM $13^{1}$,263b: béarn. terrador m. «terroir»; Lv 8,184b [sans contexte]: agasc. «Gebiet» Riscle 1483; LR sub terradou: abéarn. terrador de Boelhoo «territoire (du village) de Boueillo» $15^{\mathrm{e}} \mathrm{s}$. BaronBéarn, ib. sub besiat: terrador «territoire (d'une ville)» [s.d.] ArchBPyr.

b) terrer m., terer [1288; cop. ca. 1300] CoutMontsaunèsM 21a[16: trad. «terroir»].

FEW TERRA $13^{1}, 251 \mathrm{~b}$ : apr. terrier «propriété consistant en fonds de terre» $13^{\mathrm{e}}-14^{\mathrm{e}}$ s.; Lv 8,193a sub 5: agasc. terrer [à corr. terer] «Besitztum, Gebiet» Gir. 1305 .

1 terres $\mathrm{pl}$.

1296 [cop. $16^{\mathrm{e}}$ s.] Bayonne: Item, e pareilhement per l'arribeyre de l'Ador, tant que ledite mareye va au long e cap sus e per los costatz ... ab totz los vergers, bartes, camps e terres.

2 plaza, plaze

1256 [cop. ca. 1320] Auch: en Fontaner de Senzimon di une plaze a feus au Ramon de Laroi ...; entense la dite plaze dab las plazas den Garsie deu Fontarau e den Bernard de Saravere; de la dite plaze me faze cascun an lo dit Ramon de Laroy II sols de morlaas de feus a la feste de Martor.

3a terrador

1255 [cop. 1341] Montréal-du-Gers: establi lo predit maistre Pons que per totz temps aia honor e terrador e destreg a Montreyal en aquesta maneira: . . - [1288; 
cop. ca. 1300] Montsaunès: totas las terras del dit terrador de la Bena (v. aussi cidessous 3b).

$3 \mathrm{~b}$ terrer, terer

[1288; cop. ca. 1300] Montsaunès: .I. arpent de terra e-l terrador de la dita mayo . . . totz les terrers et ls loxs dels terers, aysi com ara les devant ditz homes de la dita vila an e tenen per tostemps.

\section{6 pièce, parcelle de terre:}

1. pecia de terra synt.f. [1030; cop. $17^{\mathrm{e}} \mathrm{s}$. (cont. lat.)] CartRéole, ArchGir 5,111 [18], pessa de terra 1290 [v.st.; cop. 13e s.] CartSMajHH 637[1140]; [s.d.; cop. $\left.13^{\mathrm{e}} \mathrm{s}.\right]$ CartSMajHH 624[1123], peiz de terra 1236 Bordeaux, RecGascL 122 [55]; pese 1183 [cop. ca. 1290; cont.lat.] CartGimC 233[53].

À rattacher à FEW TERRA $13^{1}, 251$ et gaul. *PETTIA 8,339b: apr. pessa $\mathrm{f}$. «portion de terre (pré, vigne, champ) d'un seul tenant et appartenant à un individu» Albi 1377, HPyr. Gers pèço; cf. mfr. pièce de terre «certaine étendue de terrain»; béarn. pessote de terre «lopin de terre»; LR sub tournè: abéarn. pesse de terre «pièce de terre» [s.d.] ArchBPyr.

2. trens de terra synt.m. 1234 [cop. ca. 1290] Bordeaux, RecGascL 117 [53: treus de terra, corr. par MillGloss et Lv 8,440b trens]; 1254 [cop. $15^{\mathrm{e}} \mathrm{s}$.] CartSSeurinB 222[243];223; 1259 CartSCrD, ArchGir 27,173; 1260 ChartAlbrM 220; 1263 [n.st.] CartSMajHH 357; 1264 [n.st.] ChartAlbrM 221; 1272 [n.st.] CartSCrD, ArchGir 27,168; 1279 Bordeaux, ArchGir 43,204[189]; 1281 Beychac, ArchGir 15,176 [16]; 1296 [n.st.] ChartAlbrM 78[51]; [13 ${ }^{\mathrm{e}}$ s.; cop. ca. 1400] ACoutBordB 141 [182];142[texte treus de terra, 1. trens] = Lv 8,440a; [av. 1300; cop. 1489] CoutBazasB, ArchGir 15,86[3: treus, 1. trens], trens de terre 1293 [cop. 1406] LVertBénacB 220[2].

À rattacher à FEW TERRA $13^{1}, 251$ et $23,224 \mathrm{~b}$ sub pièce: béarn. trens $\mathrm{m}$. «morceau, parcelle (de terre)»; mais cf. FEW *TRINICARE $13^{2}, 279 \mathrm{~b}$ : agasc. trens $\mathrm{m}$. «parcelle (de terre)» $13^{\mathrm{e}}-15^{\mathrm{e}} \mathrm{s}$., Lv, Mill[Gloss] et n8 qui explique $-s$ par l'influence de TRUNCEUS $\left(13^{2}, 337 \mathrm{a}\right.$ : aland. trons $\mathrm{m}$. «morceau, tronçon, éclat» 1519); Lv 8,440a/b: agasc. trens de binha 1493 InvGrim [aussi ib. 381: trens de terra], trens [treus à corr.] de terra «Stück (Land, Weinberg)» 1311 Rions, ArchGir 7,400[145: sans cont.], trens de terra 1445 SFerme, ArchGir 17,204 [sans cont.], abéarn. trentz de terre 1424 ArtBéarn [sans cont.], aland. trens de tera 1474 Mill, ArchGir 45,55 [50: sans cont.]; LR sub trens: abéarn. trentz de terre «parcelle de terre, pièce de terre» ca. 1400 [cop. ca. 1475] ForGén, ForsB[OG] 266[222: var.], ib. sub apartiences: [s.d.] ArchBPyr.

3. «morceau de terrain, partie d'un terrain»

tros de terra synt.m. [av. 1300; cop. 1489] CoutBazasB, ArchGir 15,117[88]. 
À rattacher à FEW TERRA $13^{1}, 251$ et prérom. *TRUKO- $13^{2}, 333 \mathrm{a}$ : cf. apr. trotz m. «tronçon (de lance)» lang. ca. 1210 avec n1: , Die qualität des $o$ von apr. trotz ist unbekannt. Zugrundeliegen könnte auch ein typus *trociu.; Lv 8,499b sub tros 3 «Stück, Teilstück»: abéarn. tros de terre 1484 DocSPé; LR sub tros: béarn. tros de terre «pièce de terre», ib. sub auque: abéarn. id. [s.d.] ArchBPyr.

4. «étendue de terrain plus longue que large»

correje f. $12^{\mathrm{e}}$ s. [cop. $14^{\mathrm{e}} \mathrm{s}$.] CartSordeR $119[\mathrm{RecGascL} 72$ lit correge $=$ Lv 1,379b «langes, schmales Feld»];120[144 (cont. lat.): éd. trad. «langue de terre»], correya [12 ${ }^{\mathrm{e}}$ s.; cop. ca. 1390] CartBigRC 25[9,7;11;14: ms. Pa, gloss «bande de terrain; champ étroit, tout en longueur»], correia $\left[12^{\mathrm{e}} \mathrm{s}\right.$.; cop. ca. 1390] 25[9,21;22;27: ms. Pa]; carreia [à corr.] [12 $\mathrm{s}$ s.; cop. après 1425$]$ CartBigRC [24: ms. Pb] et [12 ${ }^{\circ} \mathrm{s}$.; ca. 1250] CartBigRC [9,25: ms. B], coreia $12^{\mathrm{e}} \mathrm{s}$. [cop. ca. 1250] CartBigRC [17: ms. B], correga [ca. 1250] ActesBonnefontSH 263[586]; RecGascL 102[44] = Lv.

$\varnothing$ FEW CORRIGIA 2,1221; LR sub courreye: abéarn. correje «langue de terre, champ étroit et long» [sans indication de source].

$\diamond$ Rem. Cf. CartSordeM 222 n2 de l'acte $\mathrm{n}^{\circ}$ 144: «Une corrège est une parcelle de terre généralement longue et étroite, qui semble avoir été gagnée sur des marais», rappelant donc une courroie, une lanière.

5. «pièce de terre de petite dimension, terrain en rapport avec une habitation, espace situé autour d'une maison»

airial m. 1279 LAgC 11[27];13[43];15[53], gloss «terre cultivée».

FEW AREALIS 25,1367a corrigenda: agasc. airial $13^{\mathrm{e}} \mathrm{s}$. (addenda à ib., 169b: apr. airal «terrain voisin ou à l'entour d'une maison»); DOM 395a sub airal a); LR: béarn. ayriau: «La maison ... comprend l'ayriau et ses dépendances».

6. «pièce de terre limitée par une ou plusieurs rigoles»

arruille f. [1256; cop. $14^{e} \mathrm{~s}$.] LOrB $10=$ LR sub arroulhe avec comment. tiré de LOrBGloss.

FEW *ARRUGULA 25,343b: agasc. arruille f. «pièce de terre, ordinairement un verger, limitée par une ou plusieurs rigoles» Bayonne $13^{\mathrm{e}}$ s., LespyR [v. l'attestation ci-dessus].

7. «parcelle naturellement entourée (?)»

contorn m. 1176 [cop. ca. 1290; cont. lat.] CartGimC 123[62]; 1184 [cop. ca. 1290; cont. lat.] CartGimC 231; 1206 [cop. ca. 1290; cont. lat.] CartGimC 87[141].

FEW TORNARE $13^{2}, 74 a$ : apr. contorn $\mathrm{m}$. «lisière d'un champ» ca. 1240; [ = Lv 1,343b «Umfurchung» cite M: occit. countour «lisière d'un champ, partie que la charrue ne peut atteindre»]. 
$\diamond$ Rem. Cf. mlt. contornum 1181 [cop. ca. 1290] CartGimC 70 avec n1: «mesure agraire, ou bien champ en pente».

1 pessa de terra

[s.d.; cop. $13^{\mathrm{e}}$ s.] Bordeaux: Item altra pessa de terra que hom apera Al Giestar.

2 trens de terra

1254 [cop. $15^{\mathrm{e}}$ s.] Bordeaux: II trens de terra, deus quaus es l'uns devant los Predicadors.

3 tros de terra

[av. 1300; cop. 1489] Bazas: Item, si assi ha ung home et preng ung tros de terra ou de binha d'alcun home, ... et no fan point de carta, si l'ome lo pot leyssar, per la costuma, ny si lo senhor ly pot tole?

4 correje

$12^{\mathrm{e}} \mathrm{s}$. [cop. $14^{\mathrm{e}} \mathrm{s}$.] Sorde: A le font de l'Arribere Sobira, inter le correje Fort-Brasc de Peire-Lonke e le terre de le Faurie e le terre deu Pin.

5 airial

1279 Agen: Item en la vila de Gontaud deu hom 7 libr. d'arn. d'acapte per las vinhas, e per las maios, e pels airials ... e per los autres fios que li home de la vila teno del rei.

6 arruille

[1256; cop. $14^{\mathrm{e}} \mathrm{s}$.] Bayonne: e bi que in l'arruille qui es pres lo barad no-i aue pomer plantad negun.

7 contorn

1184 [cop. ca. 1290] Gimont [cont. lat.]: quod W. A. de Sirac et B., uxor ejus, . . . miserunt in pignus Donato abbati duos contorns de terra quos habebant a Calag, li cals contorns sunt infra serram et rivum qui exit de fonte de Larruza . . .

terrain: v. terrain couvert de ronces 707;2-1; terrain couvert d'ajoncs 747,2-1; 4-2; terrain couvert de genêts $749,1-1$; terrain couvert de jonc $1170,1-1 ; 1-3$

2707 terrain broussailleux [add. à terrain couvert de broussailles 697]:

1. brosted m. [ca. 1180; cop. ca. 1390] CensIbos, CartBigRC 34[19,31: p.-ê. «terrain broussailleux dans lequel on peut faire paître certaines bêtes» ou «le droit lui-même de faire paître certaines bêtes dans un tel terrain»].

FEW germ. *BRUST- $15^{1}, 312 \mathrm{a}$ : cf. gasc. broustè m. «lieu couvert de pousses d'arbres; taillis», Teste broustéy «fourré de broussailles»; LR: béarn. broustetz pl. «petits arbustes; broussailles».

1 brosted

[ca. 1180; cop. ca. 1390] Ibos: Aramon de Casauant. XII. d. per lo brosted. 
2708 terrain sec [add. à terre 160,3-3]:

1. sec m. 1260 Bagnères-de-Bigorre, RecGascL 31 .

$ø$ FEW SICCUS 11,586b.

$1 \mathrm{sec}$

1260 Bagnères-de-Bigorre: los secs (v. sub pré 2729,2).

2709 terre inculte, en friche [v. aussi sub pâturage 2798,6]:

1. herm m. 1143 [n.st.; cop. ca. 1310] CoutCornS [36]; 1269 [n.st.; cop. 14 s.] CartMonségurM, ArchGir 5,15; 1272 TextBigM 14[herms pl.: trad. «les vacans»; RecGascLGloss «terres désertes»]; herms pl. 1272 [cop. 1406] LVertBénacB 188; id. 1278 [cop. 1551] CartOloron, ForOlDC 100a[1]; RecGascLGloss: ca. 1290 = LR sub (h)erm; id. 1287 ArchBPyr, LR sub mentaut; [1288; cop. ca. 1300] CoutMontsaunèsM 7a, erm [ca. 1250] ActesBonnefontSH 263[586]; Brunel 342[347, gloss «non cultivé»]; erms pl. 1274 [n.st.] Mill, ArchGir 45,9[5,6]; 1281 [cop. 14 s.] DocSPéB 394, hermps pl. 1275 AnglGuyB 236b[513].

FEW EREMUS 3,237a: apr. aland. erm m. «terre inculte, lande», Lavedan herm Cordier 21, béarn. herm, Ferrère jèrm «terrain en friche»; LR sub (h)èrm «terre inculte, lande»: abéarn. herems pl. «vacants» 1520 CoutSoule, ib. sub hourastè; arcoelhe;augoer: herms pl. [s.d.] ArchBPyr.

2. pogge f. 1166 [cont. lat.] CartBerdouesC 484[718] = Lv 6,423a sub poja; 1244 [cont. lat.] CartBerdouesC 291[438] = Lv; 1252 [cop.?] Soule, RecGascL 50 = Lv.

FEW PODIUM 9,111b: agasc. pogge f. «terre en friche traversée par un chemin» [avec n6: ,Bed. steht nicht ganz fest. Wahrscheinlich handelt es sich um einen abhang, den ein weg hinaufführt. ${ }^{\prime}$ ] R 6,152; M: poujo, poutjo «étendue de pays ordinairement en friche, et traversée par une route ou un chemin, en bas Limousin»; cf. LR sub poudge: abéarn. podge «chemin» [s.d.] ArchBPyr et comment.: «un champs s'est potjat quand il est redevenu pré après qu'on ait cessé de le cultiver, qu'il ait été mis en jachère pour se reposer un temps etc.».

3. no colt synt.m. 1252 Bordères, LuchaireEt 292.

À rattacher à COLERE 2,886a.

4. «terre agricole inculte, inoccupée»

a) terra no couta synt.f. [1239] LAgC 39; 1270 Lectoure, DocGalardN 4 ${ }^{1}, 34$, terra no colta 1256 Casteljaloux, RecGascL 79, terre no coute 1260 Bagnèresde-Bigorre, RecGascL 30; 1272 TextBigM 14, terre no cote 1277 [cop.] Mill, ArchGir 45,68[26,23/24], tere no cote 1274 [n.st.] Mill, ArchGir 45,8[5,6], terra no cota 1270 Lectoure, DocGalardN $4^{1}, 32$, terra non cauta 1270 Lectoure, 
DocGalardN 4 ${ }^{1}, 32$; (causa) no caulta 1276 [n.st.; cop. $17^{\mathrm{e}}$ s.; concern. le château de Benauge (Gir.)], ArchGir 10,93[43].

À rattacher à FEW TERRA $13^{1}, 245 \mathrm{a}$ et COLERE 2,886a; LR sub cole: abéarn. no colt «(terrain) non cultivé».

b) terra buita synt.f.: terra biuta 1234 [cop. 1290] Bordeaux, RecGascL 117 [53], gloss «vide, déserte» $=\mathrm{Lv} 8,812 \mathrm{~b}$ sub voig 1 von Grundstücken: «unbenutzt, unbebaut»; 1254 [cop. $15^{\mathrm{e}}$ s.] CartSSeurinB 224[244]; (terre) buita 1260 CartSCrD, ArchGir 27,183[178: trad. «terre inculte»], (terre) buite 1283 CartSCrD, ArchGir 27,262[290], terra boita 1263 CartSCrD, ArchGir 27,226 [= Lv];227;228.

À rattacher à FEW TERRA $13^{1}, 245 \mathrm{a}$ et *VOCITUS $14,589 \mathrm{~b}$.

c) terras hermas loc.f.pl. 1246 [cop. $15^{\mathrm{e}}$ s.] CartSSeurinB 202[223]; 1252 [n.st.; cop. 15 s.] CartSSeurinB 252[270]; 1256 [cop. 15 s.] CartSSeurinB 239[261]; 1270 Lectoure, DocGalardN 4 ${ }^{1}, 32 ; 1289$ [n.st.; cop. 1667] Bordeaux [texte: nermas à corr.], ArchGir 32,192; 1291 ChartAlbrM 71; 1293 [n.st.] ChartAlbrM 245 [185], teras ermes 1256 [cop. ca. 1320] CartBlanc, CartAuchL $297=$ Lv 5,70a sub malon; RecGascL 105 [terras ermes], terres hermes 1300 Mill, ArchGir 45, 193/194[55,60]; terras heremas ca. 1274 AnglGuyB 187b[463]; culturas ermas synt. mixte lat.-gasc. [ca. 950; cop. ca. 1230] CartNoir, CartAuchL 45[47].

À rattacher à FEW TERRA $13^{1}, 245 \mathrm{a}$ et EREMUS 3,237a: apr. erm, - $a$ adj. «inculte, désert» Lv; BrunelCh; cf. aussi ib. apr. ermas [m.] «terre inculte, friche» [les contextes ci-dessus ne font pas ressortir clairement la fonction de (h)ermas en tant que subst. ou adj. À défaut d'un exemple évident de ermas $\mathrm{m}$. nous tendons à le classer comme élément des syntagmes terras acoitivadas/couttas/gazanhadas e hermas, etc.].

5. terre [a] acampir synt.f. 1258 [cop. $14^{\mathrm{e}}$ s.] LOrB $19=\mathrm{LR}$ sub acampir; 1259 [cop. $14^{\mathrm{e}} \mathrm{s}$.] LOrB $31 ; 32$.

À rattacher à FEW TERRA $13^{1}, 245 \mathrm{a}$ et CAMPUS 2,157b.

1 herm

1143 [n.st.; cop. ca. 1310] Corneillan: Los caslaas an espleit sober la terra deu seior per colt e per herm.

3 no colt

1252 Bordères: Arnaut de Laueda . . . beno ... tot lo colt e-1 no colt.

4a terra no couta

[1239] Agen: terras coutas e no coutas (terra no colta v. sub terre cultivée 2710,1).

$4 \mathrm{~b}$ terra boita

1263 Bordeaux: las terras boitas que ... tene à sa man.

4c culturas ermas, terres hermes $\mathrm{pl}$.

[ca. 950; cop. ca. 1230 (cont.lat.)]: in ipso loco dabat alodem terras, et casalas, et 1716 
vineas, et culturas ermas et condregtas. - 1300 Mimizan: vinhes, terres coitiuadas e hermes.

5 terre $[a]$ acampir

1258 [cop. $14^{\mathrm{e}}$ s.] Bayonne: Sabude cause sie qu'en A. seigner de le mazon d'Ordozon a empeinad a le Glizie de Baione . . . tot lo dezmau d'Estiei . . en fugs cubets ho en laussetaz, en terres acampides ho [a] acampir.

2710 terre cultivée [v. aussi terre labourée 2753]:

1. terra couta synt.f. [1239] LAgC 39; 1270 Lectoure, DocGalardN 4' 34; ca. 1274 AnglGuyB 187b[463]; 1291 ChartAlbrM 71[45], terra coutta 1289 [n.st.; cop. 1667] Bordeaux, ArchGir 32,192, terre coute 1260 Bagnères-de-Bigorre, RecGascL 30; 1272 TextBigM 13, terre cote 1277 [cop.] Mill, ArchGir 45,68[24], tere cote 1274 [n.st.] Mill, ArchGir 45,8[6], terra cota 1270 Lectoure, DocGalardN $4^{1}, 32$, terra colta 1256 Casteljaloux, RecGascL 79, tera colta 1256 [cop. ca. 1320] CartBlanc, CartAuchL $297=\mathrm{Lv}$ 5,70a sub malon, terra cauta 1270 Lectoure, DocGalardN 4 ${ }^{1}, 32$, terra couita ca. 1300 StatCaudecosteB, ArchGir 31,221[4]; (h)eretat culte 1294 [cop. ca. 1480] CartDax: LNoirDaxA, ArchGir 37,382;383; (causa) caulta 1276 [n.st.; cop. $17^{\mathrm{e}}$ s.: concern. le château de Benauge (Gir.)], ArchGir 10,93.

À rattacher à FEW TERRA $13^{1}, 245 \mathrm{a}$, HEREDITARE 4,410a et COLERE 2, 886a: abéarn. coler «cultiver» [= LR sub cole].

2. terra cautiuada synt.f. 1257 ChartAlbrM 46[5], terra coytiuada 1293 [n.st.] ChartAlbrM 245, terre coitiuade 1300 Mill, ArchGir 45,193[55,60], terre coitivade 1282 [cop. 1356] MartOrthB 95, terra coltivada [1288; cop. ca. 1300] CoutMontsaunèsM 7a[5]; hono[r] cultibada: honos cultibadas pl. 1276 [cop. ca. 1330] CoutFezB, ArchGir 27,356[15].

À rattacher à FEW TERRA $13^{1}, 245 \mathrm{a}$, HONOS, -OREM 4,465b et COLERE 2, 886b: abéarn. coytibar «cultiver (la terre)» avec n1, béarn. couytibá, coeytibá et ib.,887a: abéarn. cultibar «cultiver (la terre, une plante)», béarn. cultibá [= LR]; Rn 2,443b: alang. terra coltivada «terre cultivée» ca. 1420 CatApostRoma; LR sub coytibar: abéarn. terre coytivade [s.d.] ArchBPyr.

3. terra acoitivada synt.f. 1246 [cop. $15^{\mathrm{e}}$ s.] CartSSeurinB 202[223], terra acoutivada 1256 [cop. $15^{\mathrm{e}}$ s.] CartSSeurinB 239[261].

À rattacher à FEW TERRA $13^{1}, 245$ a et COLERE 2,886b: apr. acoltivar «cultiver» LvP, gasc. acoueitiouá Ad 68, Gers «cultiver avec soin»; Lavedan acoutibat «terre cultivée avoisinant les terrains incultes».

4. terre acampide synt.f. 1258 [cop. $14^{\mathrm{e}}$ s.] LOrB $19=\mathrm{LR}$ sub acampir; 1259 [cop. 14 s.] LOrB 31 . 
À rattacher à FEW TERRA $13^{1}, 245 \mathrm{a}$ et CAMPUS 2,157b; cf. LR: acampir la terre «convertir une terre en champ» [s.d.] ArchBescat.

5. terra gazanhada synt.f. 1252 [n.st.; cop. 15 s.] CartSSeurinB 252[270], terra gadanhada 1258 CartSCrD, ArchGir 27,177.

À rattacher à FEW TERRA $13^{1}, 245 \mathrm{a}$ et abfrq. *WAIĐANJAN $17,461 \mathrm{a} / \mathrm{b}$ : apr. gazanar v.a. «cultiver» Moissac 1197; Toulouse 1200, Toulouse gazanhá «labourer».

6. colt m. 1143 [n.st.; cop. ca. 1310] CoutCornS [36]; 1252 Bordères, LuchaireEt 292, cout: couts pl. 1272 [cop. 1406] LVertBénacB 188; 1278 [cop. 15 ${ }^{\mathrm{e}}$ s.] ForOl, ForsB[OG] 496[1: aussi coutz var., gloss: f.pl.], coot: cootz pl. 1278 [cop. 1551] ForOlDC 100a[1]; ForsB[OG] 496[1; gloss: f.] = Lv 1,288b «bebautes Feld» [LvP: m.]; LR sub cole et (h)èrm «terre cultivée», cot 1281 [cop. 14e s.] DocSPéB 394, coet 1278 [cop. 1460] ForOl, ForsB[OG] 496[1: coetz pl.; trad. «pâturages clos», gloss «terres en culture»], coeys pl. 1278 [cop. $15^{\mathrm{e}}$ s.] ForOl, ForsB[OG] 496[1: var.].

FEW COLERE 2,886a: apr. colt $\mathrm{m}$. «terre en culture» lang. gasc., cout Pans, aland. coot; cf. LR sub coutz: abéarn. cootz pl. «anciennement pâturages ceints de bornes servant de clôtures» 1442 .

7. coytivat m. 1278 [cop. 1460] ForOl, ForsB[OG] 496[2], coeytivat 1278 [cop. $15^{\mathrm{e}}$ s.] ForOl, ForsB[OG] 498[2: var.], quoeytivat 1278 [cop. 1639] ForOl, Fors$\mathrm{B}$ [OG] 498[2: var.], cultivat 1278 [cop. 1551] ForOlDC 102a[2]; RecGascL 55[2] $=\operatorname{Lv} 1,289 \mathrm{~b}$ «bebautes Stück Land»; LR sub cultiba «(terrain) cultivé».

FEW *COLTIVUS adj. < COLERE 2,886b: apr. coltivat $\mathrm{m}$. «terre en culture» $=\mathrm{LV}$

8. coltura f. 1176 [cop. ca. 1290; cont. lat.] CartGimC 123[62], couture $12^{\mathrm{e}} \mathrm{s}$. [cop. $14^{\mathrm{e}} \mathrm{s}$.; cont. lat.] CartSordeR $120=\mathrm{LR}$ sub culture «terre cultivée, champ», cooture 1272 [cop. 1406] LVertBénacB 188 , culture $12^{\mathrm{e}} \mathrm{s}$. [cop. $14^{\mathrm{e}} \mathrm{s}$.; cont. lat.] CartSordeR 120; RecGascL 73 = Lv 1,290a «bebautes Feld» [sans cont.]; 1258 CartBlanc, CartAuchL 307[76]; RecGascL $109=$ Lv.

FEW CULTURA 2,1504a: apr. coltura «champ labouré, terre cultivée», abéarn. [à corr. aland.] couture $12^{\mathrm{e}} \mathrm{s}$. [v. l'attestation ci-dessus].

9. arecolter m. $12^{\mathrm{e}} \mathrm{s}$. [cop. $14^{\mathrm{e}} \mathrm{s}$.; cont. lat.] CartSordeR 120; RecGascL 72, gloss «terrain cultivé» = Lv 7,106b sub recoltier ?; LR sub arrecolter «(où l'on fait des récoltes), champ cultivé».

FEW COLLIGERE 2,903a: abéarn. arrecolter [corr. aland. arecolter, v. cidessus] «champ cultivé».

10. condreis m.pl. 1272 TextBigM 14 [trad. «pâturages»] = Lv 1,319b sub condrech «bebautes Stück Land». 
FEW *CONDIRIGERE 2,1022a: apr. condrech $\mathrm{m}$. «. . .; terre en culture» $13^{\mathrm{e}}-14^{\mathrm{e}} \mathrm{s}$.

11. «terre à cultiver, terre en culture»

coltiu m. [1288; cop. ca. 1300] CoutMontsaunès v. trayre de herm au coltiu sub défricher 2712,2.

$\varnothing$ FEW *COLTIVUS adj. < COLERE 2,886b: cf. Lézignan coutiu «terre en friche, non cultivée» RLR 40, 321, Agen couütioü «terre qui donne des légumes de bonne cuisson».

\section{1 terra colta}

1256 Casteljaloux: totas les terras del dit log, coltas e no coltas (terra couta v. sub terre inculte, en friche $2709,4 a$ ).

\section{2 hono[r] cultibada}

1276 [cop. ca. 1330] Fezensaguet: Si home o femma sera trobat o trobada en las honos cultibadas, despuys que seran defensadas per lo solet entrament, pague cascun o cascuna IIII. dines tolsas per justicia (terra coltivada v. sub défricher 2712,2).

3 terra acoitivada

1246 [cop. $15^{\mathrm{e}}$ s.] Bordeaux: en Arnaut d'Espanha, lo cavors, . . . arreconogo e fermament autreiet que totas las terras acoitivadas e hermas, landas ... ten de la gleisa Sen-Seurin.

\section{4 terre acampide}

1259 [cop. $14^{\mathrm{e}} \mathrm{s}$.] Bayonne: E d'autre part ahos pres em peinhs la mentahude Glizie de Baione ... tot lo dezmau d'Estiei ab tot sons tinhs, e ab sos dretadges or que-s sian ... en terres acampides, e acampir (v. aussi sub terre inculte, en friche 2709,5).

\section{5 terra gazanhada}

1252 [n.st.; cop. $15^{\mathrm{e}} \mathrm{s}$.] Bordeaux: binhas et terras gazanhadas et hermas, boscs, et landas.

6 colt

1143 [n.st.; cop. ca. 1310] Corneillan: los caslaas an espleit sober la terra deu seior per colt e per herm (v. aussi terre inculte, en friche 2709,3).

7 coytivat

1278 [cop. 1460] Oloron: en los coytivatz ayen erbe et pastenc obs de lors jumentz.

\section{8 coltura}

1176 [cop. ca. 1290] Gimont [cont. lat.]: predicti dederunt Umberto abbati totam medietatem de la coltura de Rodas.

9 arecolter

$12^{\mathrm{e}} \mathrm{s}$. [cop. $14^{\mathrm{e}} \mathrm{s}$.] Sorde [cont. lat.]: tres arecolters inter terram de Focui. 


\section{1 terre/terrain défriché(e):}

1. eychart $\mathrm{m}$. [1280; cop. $17^{\mathrm{e}} \mathrm{s}$.] CoutSeixP 8[49]; sarta [ou asarta] f. [forme douteuse] 1247 [cop. 13e s.] RecFéodD, ArchGir 3,3[4: la sarta], ArchGir11Gloss «défrichement ?»; AnglGuyB 166a[421: l'asarita]; ysadies pl. [forme corrompue ?] 1256 [cop. ca. 1320] CartBlanc, CartAuchL 297; RecGascL 105.

FEW *EXSARTUM 3,318b: apr. eisart «défrichement; terre défrichée», cf. ib.: Castillon echart(as) «terrain rocheux», ib.,318b: apr. eisartada «terre défrichée» et 319a comment.: mlt. exartus, asartus «gereutetes Land, Rodung» Piémont.

$\diamond$ Rem. Cf. DC 7,314b: mlt. sartum, sartus: «terra dumetis purgata, et in culturam redacta» et ib.,313c sub sartarii: „Homines, qui fundos, Sarta nuncupatos, possidebant ....

2. artigua f.: artiuga 1177 [cont. lat.] Escale-Dieu, RecGascL 11[10]; 1257 [n.st.] CartSCrD, ArchGir 27,253[274]; 1258 [cop. ca. 1290] CartFronsacTD, ArchGir 38, 31; 1260 ChartAlbrM 220; 1276 [n.st.] Bordeaux, ArchGir 43,169[172], artiga 1252 [cop. 1260] Bordeaux, ArchGir 23,9; 1252 [n.st.; cop. 15e s.] CartSSeurinB 253[270]; 1258 [cop. ca. 1290] CartFronsacTD, ArchGir 38,30;31[19]; 1271 [n.st.] Cérons, ArchGir 3,272; ChartAlbrM 61[30]; 1284 ChartAlbrM 139; 1289 [cont. lat.] Libourne, ArchGir 10,108[63].

FEW prérom. *ARTIKA 25,387b: agasc. artiga f. «terre défrichée» Gir. 1258-1289, Baldinger, artigue Ossau hap. $15^{\mathrm{e}}$ s., DiccEtCat 1,439; cf. LR: béarn. artigue «pré» [v. Lv 4,115b sub gerba: abéarn. artiga et prat 1486 DocSPé 1895,468] et Palay: artigà «terre défrichée, convertie nouvellement en pâturage», v. aussi FEW 25,389b/390a n14.

3. artigal m. 1166 [cop. ca. 1290; cont. lat.] CartGimC 156[127]; 1179 Montsaunès v. far artigal sub défricher 2712,1; Pfister, Vox 18,280, artigau 1233 [cop. 1406] LVertBénacB 181[19]; 1258 CartSCrD, ArchGir 27,176; 1300 [cop. 1356] MartOrthB 180.

FEW prérom. *ARTIKA 25,387b: agasc. artigal m. «terre défrichée» Comminges 1179, Brunel; cf. Via Domitia 22,23 avec n5, artigau Bord. 1258, Baldinger avec n6, VGer artigau 1698, Dinguirard 106, Arrens artigáu, Bazas artigau «pièce de terre défrichée»; LR: abéarn. $i d$. «terre défrichée, défrichement» 1520 CoutSoule.

4. artigon $\mathrm{m}$. [ou à lire artigou ?] 1247 [cop. 15 s.] CartSSeurinB 200.

ø FEW prérom. *ARTIKA 25,387b.

1 sartalasarta, eychart

1247 [cop. $13^{\mathrm{e}} \mathrm{s}$.] Blanquefort: Na Mabila, ... reconogo que n'Arnaut de Blancafort son maritz a mes et pagat, et de sos propris deners, en las obras que a fet en castet de Blancafort et e-us molins en la sarta [l'asarta?] de Penhs, que en Galhard 
Colomb ... tene en feu feaument. - [1280; cop. $17^{\mathrm{e}}$ s.] Seix: Item qui desclauga o eychart $\mathrm{o}$ vigna, pague $\mathrm{XX}$ diniers per justicia.

2 artiga

1252 [cop. 1260] Bordeaux: que l'auantdeyt capitre a dat . . . aus auantdeyts homes et a lur orden ... tot lo remanen de l'auantdeyta palu de Cadaujac . . . exceptadas las auandeytas mille sadons ... et l'artiga de Noailhan.

\section{3 artigal}

1166 [cop. ca. 1290] Gimont [cont. lat.]: illas terras que vocantur artigals de Raimun Boz.

4 artigon

1247 [cop. $15^{\mathrm{e}} \mathrm{s}$.] Bordeaux: tota aquera boria . . . entre la planta n'Arnaut-W. Aimeric, d'una part, e l'artigon que fo W. Arrevet, d'autra part.

\section{2 défricher:}

1. far artigal synt.verb. 1179 Montsaunès, RecGascL 5[4]; Brunel 160[172,6, gloss: artigal «défrichement, essart»].

FEW prérom. *ARTIKA 25,387b: agasc. far artigal «entreprendre un défrichement» Comminges 1179, Brunel [v. l'attestation ci-dessus]; cf. Via Domitia 22,23, far artigau Soule 1520, CoutGén 4, 985; var. artigal éd. 1692, LespyR.

2. «terre inculte qui sera cultivée, qui est rendue propre à la culture»

trayre de herm au coltiu synt.verb. [1288; cop. ca. 1300] CoutMontsaunèsM $7 \mathrm{a}[5$; trad.: «tirer de herm à culture»].

À rattacher à FEW TRAHERE $13^{2}, 177$ etc., FEW EREMUS 3,237a et *COLTIVUS adj. < COLERE 2,886b; Lv 8,362b sub 29: cf. traire terra «Land bebauen»: agasc. treyre trens de terre 1489 Puybarban, terras treitas et a treire 1318 Castelnau-de-Médoc, aland. teres treytes et a trer 1445 Chalosse.

1 far artigal

1179 Montsaunès: E, si la mason de Montsalnes fazie artigal e-l dezmari, ne nuls om per lor, que dels.V. ans non deuen dar dezma.

2 trayre de herm au coltiu

[1288; cop. ca. 1300] Montsaunès: totas las terras coltivadas o aquelas que trayran de herm au coltiu.

\section{3 fixation des limites de terre:}

1. esterminement de terre synt.m. 1265 [cop. 1439] ChartSSeverPC 432. 
À rattacher à FEW TERRA $13^{1}, 251 \mathrm{a}$ et TERMINUS $13^{1}, 240 \mathrm{a}$ : cf. aland. estremiá v.a. «limiter», béarn. estermiá «borner».

1 esterminement de terre

1265 [cop. 1439] SSever: si [querele y] abié enter En Guitar et los pobledors . . de la ladite terre per areson d-esterminement de terre...

\section{4 limite (d'un terrain, d'un champ):}

1. «limite (d'une propriété)» couffi m. [1265; cop.] CoutSarrant, CoutGersB 40.

FEW CONFINIS 2,1035a: apr. confin sg. «limite» LvP, gasc. couhis pl. «confins» Ad 130; Gers couhin sg. «borne», Vic-Bilh couhî.

1 couffi

[1265; cop.] Sarrant: se aucun habitant ... mudaue ou darrigaue los couffis ou boussoles entre deuos pocessios que sio puignit.

\section{5 attenant, limitrophe:}

1. «attenant à (une propriété)» confrontat p.p. 1267 [cop. 14 ${ }^{\mathrm{e}}$ s.] CartSCrD, ArchGir 27,297.

FEW mlt. CONFRONTARE 2,1045b: cf. apr. confrontar «v.n.r. confiner; v.r. borner», prov., lang., Puiss. counfrountá «être limitrophe»; Rn 3,402b: agasc. (prat) confrontat p.p. «confronté» ca. 1465 Bordeaux.

1 confrontat p.p.

1267 [cop. $14^{\mathrm{e}} \mathrm{s}$.] Bordeaux: e plus sobre totz aquetz autres trens de binhas qui son aqui medis, ayssi cum son entre certanas confrontations; totz losquaus trens de binhas son confrontatz ab lo feu de mossenhor l'abat deudeyt mostey.

\section{6 terrain attenant à une propriété, propriéte limitrophe:}

1. afrontatio $[\boldsymbol{n}$ ] f.: afrontatios pl. 1260 Bagnères-de-Bigorre, RecGascL 31 [gloss: «limite»]. - [LR sub affrount: à biffer affront $\mathrm{m}$. «anciennement, partie (du terrain) contiguë» LRougeOssau $=1278$ [cop. $15^{\mathrm{e}}$ s.] CartOssauT 59 qui lit la Fron (gloss: La Fron, La Ron(t): Bois de Pau ou Forêt de Bastard); DOM 287a sub afron $b)$ à corr.].

FEW FRONS, -TIS 3,821b: apr. afrontazon «limite» [=LvP; Rn 3,402a «confrontation, confin, limite»: alang. afrontazos f.pl. «confrontations» 1234 Toulouse; DOM sub [afrontason] 288a]; cf. LR sub affrountatiou: abéarn. 
affrontation «confrontant, ce qui confronte» [source non indiquée]; DOM 287a sub [afrontacion] «limite, partie limitrophe [d'un terrain]».

2. confrontation f. 1267 [cop. 14 se s.] CartSCrD, ArchGir 27,297; 1293 [cop. 1406] LVertBénacB 221[2].

FEW mlt. CONFRONTARE 2,1045b: apr. confrontacion «limite» Rn; Lv; Doc 36; Lv 1,323b: agasc. confrontation «Grenze» 1334 [cop. après 1336] EtBay.

\section{1 afrontatio $[n]$}

1260 Bagnères-de-Bigorre: totes las terres coutes e no coutes, $\ldots$. ab lors estremitaz e afrontatios.

\section{2 confrontation}

1293 [cop. 1406] Lavedan: Pels diits diners los beno la diite terre . . . he liura l'ag $\mathrm{ab}$ fust e ab terre, ab tots los entradz, e-ls exids e las confrontations, e la[s] apertiensas (v. aussi sub attenant, limitrophe 2715,1).

\section{7 délimiter une terre, un terrain:}

1. esdegar v.tr. 1277 ChartSSeverPC 522.

FEW DECUS 3,27a: apr. esdegar «borner» avec n2: Vgl. mlt. esdegatum «limité» Gascogne, R 35, 320[= CartGimC 11: 1169 [cop. ca. 1290] «détérminé, borné»]: mot gascon affublé d'une terminaison latine; LR sub esdegament: abéarn. esdegar «borner» [source non indiquée].

$\diamond$ Rem. V. mlt. esdegare dans Mireille Mousnier, «Mesurer les terres au Moyen Âge. Le cas de la France méridionale», dans Histoire et Sociétés Rurales, vol 22, 2004/2, 29-63: p. 32.

1 esdegar

1277 [SSever]: ... general poder al noble baron ... de esdegar ladite terre del Mortis ab la nostre.

\section{8 limiter un terrain par une ou plusieurs rigoles:}

1. arruillar v.tr. [1256; cop. $14^{\mathrm{e}} \mathrm{s}$.] LOrB $8=$ LR [d'après LOrBGloss] sub arrulhar «limiter par une rigole ou des rigoles une pièce de terre pour la cultiver»; 1259 [cop. $14^{\mathrm{e}} \mathrm{s}$.] LOrB 12 [cependant RecGascL 85, gloss «canaliser, creuser» $=\mathrm{Lv} 7$, $372 \mathrm{~b}$ sub rolhar avec renvoi à ib.,372a sub rolha]. - [V. aussi arruille «pièce de terre limitée par une ou plusieurs rigoles» sub pièce, parcelle de terre 2706,6].

FEW *ARRUGULA 25,343b: agasc. arrulhar v.a. "creuser une rigole, limiter par une ou plusieurs rigoles une pièce de terre à cultiver» Bayonne $13^{\mathrm{e}} \mathrm{s}$, LespyR [sub arrulhar, v. l'attestation ci-dessus]. 
2719 rigole:

1. arruille f. [1256; cop. $14^{\mathrm{e}} \mathrm{s}$.] LOr v. far arruille sub creuser une rigole 2720,1; 1259 [cop. $14^{\mathrm{e}} \mathrm{s}$.] LOrB 11[arruille uberte];12[.III. arruilles] [v. aussi far far arruille sub 2720,1]; RecGascL 84;85 [gloss «canal, petit ruisseau»] = Lv; LR sub arroulhe [corr. arrulhe], arroille 1296 [cop. 16 ${ }^{\mathrm{e}} \mathrm{s}$.] EtR 2,81[2], arolha 1291 [cop. 17 s] ChartAlbrM 241[184].

FEW *ARRUGULA 25,343a: abéarn. [à corr. agasc.] arruille f. «petit canal ou ruisseau» Bayonne 1259 [v. l'attestation far far arruille ci-dessus], Lv 7,372; LespyR avec n1 [«Déjà enregistré ici 10,423b, RIVUS.»], arrulhe [à corr. arruille] hap. Bayonne $13^{\mathrm{e}} \mathrm{s}$. [1259 LOrB, v. l'attestation arruille uberte], LespyR, aroilhe Bayonne 1304, Lv 7,372, agasc. rulha Bordeaux 1416 [à corr. 1420], ibid. [aussi gascon latinisé rolha 1360 ObitBord], béarn. arroulhe «rigole, canal, fossé», land. arrouille «rigole, petit fossé, égout», Bazas arr $(o)$ ulhe «fossé, rigole, égout»; LR sub arroulhe: abéarn. arrolhe «canal» 1520 CoutSoule.

2. calars m.c.s. [12 s.; cop. ca. 1390] CartBigRC 25[9,19; gloss «rigole, fossé»].

$ø \mathrm{FEW} * \mathrm{CALA} 2,50 \mathrm{~b}$ : cf. land. cale «rigole; creux d'un sillon». V. le comment. dans CartBigRCGloss sub calars.

1 arroille

1296 [cop. $16^{\mathrm{e}}$ s.] Bayonne: ... ab lo flot de le mareye per esters, aigues et arroilles...

2 calars $\mathrm{c} . \mathrm{s}$.

[12 s.; cop. ca. 1390] Barèges: Ed hic sunt scripti homines qui tenent terras qui debent dare equam comiti ... Arramon Bet lo calars d'Agasalsa e-1 serr e-1 prat e bigna ...

\section{0 creuser une rigole:}

1. «(faire) creuser une rigole»

far arruille synt.verb. [1256; cop. $14^{\mathrm{e}} \mathrm{s}$.] LOrB $8=$ LR sub arroulhe; far far arruille 1259 [cop. 14 ${ }^{\mathrm{e}} \mathrm{s}$.] LOrB 11; RecGascL 84[36] = Lv 7,372a sub rolha.

À rattacher à $\mathrm{FEW} *$ ARRUGULA 25,343a.

2721 fossé qui sert de limite aux terrains [add. à fossé 188,1-1;2-1]:

1. fossat m. 1244 [cop. $15^{\mathrm{e}} \mathrm{s}$.] CartSSeurinB 296[315]; 1263 CartSCrD, ArchGir 27, 228; 1279 Bordeaux, ArchGir 43,204[189]; s.d. [ca. 1280] Bordeaux, ArchGir 43, 211, ffossat 1263 CartSCrD, ArchGir 27,228. 
FEW FOSSATUM < FOSSA 3,740b: béarn. foussat «fossé» [= LR: abéarn. fossat, foussat [s.d.] ArchBPyr], Teste houssat; Rn 3,348a: apr. esser claus de fossatz «être clos de fossés» ca. 1190 BertBorn.

2. barat m. 1273 [cont. lat.] AnglGuyB 186b[461]; 1274 AnglGuyB 35a, barad 1294 ArbitrTarbesR 9b[9], valat ca. 1300 StatCaudecosteB, ArchGir 31,230[47];234 [61].

FEW VALLIS 14,137a/b: agasc. barat $\mathrm{m}$. «fossé» avec n6;7, Gers id. «id.»; Lv 8,568a sub 1 «Graben» renvoie à ib. 3,300a sub estan 2 et ib. 7,568a sub valadier: agasc. barat 1442 et 1444 CConsRiscle; LR: béarn. barat «fossé», ib. sub bocabarat;pau: abéarn. id. [s.d.] ArchBPyr.

1 fossat

1279 Bordeaux: tot aquet trens de terra ab sos fossatz.

2 barat

1273 [Dax]: la maitat de tot l'afar de Bordessolos, . . . loquau afar s'esten de Bordessoles trouqu'au Sequos,... e de W. Son trou barat d'Agreu e dou barat deu Greu trou Sanct Juzan.

\section{2 entourer d'un fossé:}

1. baradar v.tr. s.d. [ca. 1280] Bordeaux, ArchGir 43,210[193] = Lv; 1296 [cop. $17^{\mathrm{e}} \mathrm{s}$.] ChartAlbrM 80.

FEW VALLIS 14,137b: agasc. baradar v.a. «entourer de fossés», béarn. baradá; Lv 8,567a/b sub valadar: aland. baradar ca. 1345 EtDax, LNoirDax, gloss «entourer d'un fossé, d'un tertre», abéarn. 1424 ArtBéarn (sans cont.) [= LR sub barada: barradat «(champ, enclos) entouré d'un fossé»].

2. baradegar v.tr. s.d. [ca. 1280] Bordeaux, ArchGir 43,211.

FEW VALLIS 14,137b: agasc. baradeiar v.a. «entourer de fossés»; Lv 8, 567b sub valadejar 1 «mit einem Graben umgeben» 1304 CoutAstafort.

\section{1 baradar}

1296 [cop. $17^{\mathrm{e}} \mathrm{s}$.] Bayonne: vulh, que tant cum io fids baradar de la terre deus homis de Podensag au loc on las forquas eren.

2 baradegar

s.d. [ca. 1280] Bordeaux: que aga fossatz VI pes de prigon e que ac pusca baradegar ... camyn de Trageit XX pas o menchs qui ja ne sia baradegat.

\section{3 barrière:}

1. «barrière de bois délimitant un lieu, qui sert de clôture à un champ (?)» barerra f. [12 $\mathrm{s}$.; cop. ca. 1390] CartBigRC 25[9,23: ms. Pa], barrera $\left[12^{\mathrm{e}} \mathrm{s}\right.$.; 
cop. ca. 1250] CartBigRC [9,26: var. B], barrere 1300 [cop. 1356] MartOrthB 180 .

FEW *BARRA 1,256a: béarn. barrère «barrière»=LR: la barrère deu camp «la barrière (qui ferme l'entrée) du champ». Cf. comment. dans CartBigRCGloss: „«barrière; clôture» (s'agirait-il, par extension de sens, d'un champ clôturé ou d'une barrière donnant accès à un terrain doté d'un statut particulier?) ${ }^{e}$.

1 barerra

[12 s.; cop. ca. 1390] Bigorre: Arramon de Porta Alta la baregla e la correia de Sancta Maria, lo plantat e la barerra e notz anclosa e baregla e So Casas.

\section{4 borne:}

1. bridana f. 1246 [cop. $15^{\mathrm{e}}$ s.] CartSSeurinB 157;158[188], bidana 1273 [cop.] ChartAlbrM 224.

FEW sub borne $22^{2}, 38$ b: agasc. bridana f. «borne» Gir. 1388 [corr. 1338, ArchGir 6,185]; Bord. 1414, 1415, blidana Bord. 1452, bidana Gir. 14 s., 1494, bidama Gir. 1466.

1 bridana

1246 [cop. $15^{\mathrm{e}}$ s.] Bordeaux: E lo camins qui entra la enz en la palu qui es bert au bordiu W. Gasc es camins comunaus trusqua a la bridana 'n Aramon de Soz-loMur.

cc) Les travaux des champs

1. L'attelage

attelage: v. sub charrue 2756,2

2725 soumis au joug:

1. juer adj.: iuer 1296 [n.st.; cop. 1406] LVertBénacB 205[1;8, gloss «habitué au joug»].

$ø$ FEW JUGUM 5,61b etc.

1 juer

1296 [n.st.; cop. 1406] Tarbes: Per cada boeu iuer que aien, lo donen, cade an, V ss. de boos morlas. 


\section{6 bouvier:}

1. boer m. [av. 1223; cont. lat.] CartMorl, RecGascL 46[25], boyhe[r]: boyhes pl. [1288; cop. ca. 1300] CoutMontsaunèsM 2a.

$\varnothing$ FEW BOS 1,445b; LR sub boè;boalhe: abéarn. boer «bouvier, qui conduit, qui garde les bœufs» [s.d.] ArchBPyr.

1 boer

[av. 1223] Morlaàs [cont. lat.]: domus Sancii boer (boyhes pl. v. sub berger 2806,2).

\section{7 aiguillon (du bouvier):}

1. agulho[n] m.: agulhos pl. 1265 [cop. 17 ${ }^{\mathrm{e}}$ s.] CoutLarrC 121[15]; id. 1270 [cop. 1455] CoutMontoussD 650[43].

FEW *ACULEO 24,123b: apr. agulion m. «pointe de fer au bout d'un bâton servant à piquer les bœufs» BBorn, Rn, agulhon 1390 Pans.

1 agulho[n]

1265 [cop. $17^{\mathrm{e}} \mathrm{s}$.] Larrazet: E-l faure que tendra la fargua deu ... far los agulhos e-ls ferramens tots aquels que a l'araire s'aperteno.

2. La fenaison

2728 prairie [v. aussi sub prairie à foin 2741]:

1. pradairia f. 1256 Casteljaloux, RecGascL 79.

FEW PRATUM 9,334a: apr. pradaria f. «grande étendue de prés» $13^{\mathrm{e}}-14^{\mathrm{e}} \mathrm{s}$., Rn; Jaufre; LR sub praderies: béarn. pradaries pl. «prairies» 1583 Salette.

2. prada f. [1264; n.st.] ChartAlbrM 222.

FEW PRATUM 9,334a: apr. prada f. «prairie», Arrens id., Caut. béarn. prade $[=\mathrm{LR}]$.

1 pradairia

1256 Casteljaloux: boscs e boscadges, pradz e pradairias.

2 prada

[1264; n.st.] Gironde: e una sadon de prat qui es en la prada de Roqer, entre-u prat deu senhor de Roqer, d'una part, e le W. de Larua, d'autra.

2729 pré:

1. pratm. [12e s.; cop. ca. 1390] CartBigRC 25[9,14;19]; 1238 [vid. 1278; cop. 14e s.] 
PrivEDM[D], ArchGir 3,123; [ca. 1238; cop. ca. 1390] CartBigRC 105[71,82: ms. $\mathrm{Pa}$; [1239] LAgC 39; 1247 [cop. 15 ${ }^{\mathrm{e}}$ s.] CartSSeurinB 195;213; 1252 [n.st.; cop. 1294] ChartAlbrM 86[55]; 1255 [cop. 1341] CoutMontrOG 129[8]; 1258 CartSCrD, ArchGir 27,175[173]; 1262 [cop. 16 ${ }^{\mathrm{e}}$ s.] Bordeaux, ArchGir 53,2; 1263 [n.st.; cop. $13^{\mathrm{e}}$ s.] CartSMajHH 357; [1264; n.st.] ChartAlbrM 222; 1265 [n.st.; cop. 1291] ChartAlbrM 89[60]; 1270 Lectoure, DocGalardN 4 ${ }^{1}, 32 ; 34 ; 1270$ [cop. 1287] CoutSauve, CoutGersB 188[23]; 1274 [cop. 1406] LVertBénacB 120; 1276 [n.st.; cop. $17^{\mathrm{e}}$ s.: concern. le château de Benauge (Gir.)], ArchGir 10,93[43]; 1278 [cop. 14 s.] CartBeaumontM 3; 1279 LAgC 15[53]; 1280 Bordeaux, ArchGir 25,2; [1288; cop. ca. 1300] CoutMontsaunèsM 5a[3];6a[3;4]; 1289 ChartAlbrM 68[43]; 1289 [cop. 14 s.] Monségur, ArchGir 5,29;31[11]; [ca. 1290] HommVayresD, ArchGir 8,43; 1294 [cop. ca. 1480] CartDax: LNoirDaxA, ArchGir 37,382; 1298 Villefranche [Gers], ArchGir 29,377; 1300 [cop. 1466] CoutCastBouz, Monl 6,101; ca. 1300 StatCaudecosteB, ArchGir 31,221[4], praz pl. 1162 [cop. ca. 1290; cont. lat.] CartGimC 439[111], prad sg. ca. 1190 [cop. 18 s.] ActesBonnefontSH 258 [573]; 1196 [cop. ca. 1290; cont. lat.] CartGimC 368[121]; [12 ${ }^{\mathrm{e}}$ s.; cop. ca. 1390] CartBigRC 25[9,10]; [ca. 1238; cop. après 1425] CartBigRC [71,80: var. Pb]; 1256 Casteljaloux, RecGascL 79; 1256 [cop. ca. 1320] CartBlanc, CartAuchL $297=\mathrm{Lv}$ 5,70a sub malon; 1272 TextBigM 14; 1272 [cop. 1406] LVertBénacB 188; 1296 [cop. $17^{\mathrm{e}}$ s.] ChartAlbrM 80, prait: praitz pl. 1247 [cop. $15^{\mathrm{e}} \mathrm{s}$.] CartSSeurinB 194; pratis 1270 Lectoure, DocGalardN $4^{1}, 34$.

FEW PRATUM 9,333b: adauph. pra, prat $\mathrm{m}$. «petite étendue de terre où l'on fauche l'herbe»; Lv 4,115b sub gerba: abéarn. prat 1486 DocSPé 1895, 468; LR: id. «pré» [s.d.] ForsNav, ib. sub oun 2: [s.d.] ArchBPyr.

2. berd m. 1260 Bagnères-de-Bigorre, RecGascL 31, gloss «endroit vert».

$ø$ FEW VIRIDIS 14,508a: cf. frcomt. mettre au vert «envoyer le bétail au pâturage au printemps»».

3. «ensemble des herbes qui forment le gazon des prés et des pâturages; prés, pâturages» [add. à herbe 1021,1-1]

erbas f.pl. ca. 1140 [cont. lat.] ActesBonnefontSH 53[4]; [1288; cop. ca. 1300] CoutMontsaunèsM 8a[6], erbes 1265 Mill, ArchGir 45,4[2,5].

FEW HERBA 4,404b: cf. norm. herbes pl. «pré, pâturages»; Lv 4,115b sub gerba: abéarn. erbas f.pl. 1486 DocSPé [pratz et erbas].

$\diamond$ Rem.: Cf. mlt. herbe sive herbagia 1245 ActesBonnefontSH 146[334].

1 prad

1196 [cop. ca. 1290] Gimont [cont. lat.]: de la carrera que vadit al prad de l'arrival entro ad vineam Arnaldi Abrini (v. aussi sub prairie 2728,1; prat sub 2728,2; faucher 2735,2).

2 berd

1260 Bagnères-de-Bigorre: E nos auant dit en W. Foert autrejam e coofirmam 1728 
aqued doatiu, . . . asi com nostre maj na Comdor l'aue dad e confermad . . . so es asaber homes e femnes . . los arbes fruit fazentz e no fazentz, los secs e-us berdz, las aigues e-us molis.

3 erbes $\mathrm{pl}$.

1265 MMarsan: nos, en P. de Sent-Aubin, ab auctoritad ... e ab espres cocentiment de la done n-Aricarde, nostra molher, . . a an benud e liurad e autreyad . . . a madaune na Mathe ... tote aquere terre e tot aqued loc, bocs e augues e erbes.

\section{0 transformer en prairie:}

1. apradar v.tr. 1262 [cop. $16^{\mathrm{e}}$ s.] Bordeaux, ArchGir 53,3.

FEW PRATUM 9,335b: Gers, Arrens, béarn. apradá v.a. «mettre (une terre) en pré»; LR sub aprada: abéarn. apradar «mettre une terre en nature de pré» [s.d.] ArchBPyr.

\section{1 apradar}

1262 [cop. $16^{\mathrm{e}}$ s.] Bordeaux: que si en Guitart de Borc o suy her, o suy feuater bolon o poden de la medissa palu que sere au medis en Guitart aissi cum es auandeyt a pradar [1. apradar], o far aubaredas, o cauzas obrarias, o vinhas.

\section{1 faux:}

1. «(corvée de) faux»

fals f. ca. 1180 [cop. ca. 1390] CensIbos, CartBigRC 34[19,10;16;17: ms. Pa], falz ca. 1180 [cop. ca. 1250] CensIbos, CartBigRC [19,10;16: var. B], fahls ca. 1180 [cop. après 1425] CensIbos, CartBigRC [19,9: var. Pb], faus 1203 [cop. 1345] CoutSGaudens v. segar (erba) ab faus sub faucher 2735,2.

FEW FALX 3,404a: apr. fals «faux», béarn. haus avec n5 [=LR: selon FEW plutôt «faucille»]; cf. Lv 3,404a sub 2: agasc. fals «faucille ?» $14^{\mathrm{e}}$ s. CoutGont; LR sub haus: abéarn. fautz (faus) pl. «faux» [s.d.] ArchMontaut.

$\diamond$ Rem. En ce qui concerne les attestations dans CensIbos v. le commentaire dans CartBigRCGloss: «Il s'agit à la fois, semble-t-il, de l'obligation d'apporter sa faux et de la corvée nécessitant l'usage de cet outil».

2. dail m. 1268 Gabarret, RecGascL 87.

FEW *DACULU, -A 3,2b: apr. dalh «faux».

1 fals

ca. 1180 [cop. ca. 1390] Ibos: e forca e fals e flaget (faus v. sub faucher 2735,2).

2 dail

1268 Gabarret: . . . a ab lacal maison lo dit prior a liurad e autreiad al dit en Guiraut . . . I. lambrois, e .I. dail, e .IIII. arresteds de fer. 


\section{2 aiguiser:}

1. agusar v.tr. 1265 [cop. $17^{\mathrm{e}} \mathrm{s}$.] CoutLarrC 121[15]; 1270 [cop. 1455] CoutMontoussD 650[42]; [1288; cop. ca. 1300] CoutMontsaunèsM 53[50], agudar 1270 [cop. 1455] CoutMontoussD 650[42;43].

FEW *ACUTIARE 24,125b/126a: apr. aguisar v.a. «rendre aigu, tranchant» $13^{\mathrm{e}}-15^{\mathrm{e}}$ s., Rn; Pans 5, gasc. agusá «aiguiser» 1567, béarn. id. [= LR]; cf. DOM 341b sub agusar 1a.

1 agusar

[1288; cop. ca. 1300] Montsaunès: Item, volem qu el [1. que-1] fabre de la dita vila de Monsaones als deuant ditz habitans ... fassa ... agusar lor relhas e coutes am lo propri carbo del di fabre.

\section{3 droit perçu pour l'aiguisage des outils agricoles:}

1. agusadura f. 1270 [cop. 1455] CoutMontoussD 650[42].

FEW *ACUTIARE 24,126b: apr. aguzadura «droit perçu sur ceux qui aiguisent» LvP; cf. DOM 341a: alang. 13 $\mathrm{s}$. [texte lat.].

1 agusadura

1270 [cop. 1455] Montoussin: los senhos seu levan per cascun parelh de boeus per las agusaduras una aymina de blat.

\section{4 celui qui fait aiguiser ses outils agricoles, le soc de sa charrue:}

1. relhadie $[\boldsymbol{r}]$ m. 1270 [cop. 1455] CoutMontoussD 650[42] = Lv 7,202b «derjenige, der seine Pflugschar oder andere landwirtschaftliche Werkzeuge schärfen lässt».

ø FEW REGULA 10,218a.

\section{5 faucher:}

1. «faucher l'herbe d'un pré»

dalhar (prat) v.tr. [ca. 1238; cop. ca. 1390] CartBigRC 105[71,82: ms. Pa]; 1278 [cop. $15^{\mathrm{e}} \mathrm{s}$.] CartOssauT 57 = LR sub dalha, dalar [ca. 1238; cop. après 1425] CartBigRC [71,80: var. Pb].

FEW *DACULU, -A 3,3a: Teste daillà «faucher»; Lv 4,115b sub gerba: abéarn. dalhar 1486 DocSPé; LR sub augue 2: id. [s.d.] ArchBPyr, ib. sub plouye: béarn. 1583 Salette.

2. «couper de l'herbe à la faux»

segar (erba) ab faus loc.verb. 1203 [cop. 1542] CoutSGaudensM 46[58]; se1730 
gar (herba) v.tr. 1289 [cop. 14e s.] Monségur, ArchGir 5,29;30;31[11].

À rattacher à FEW FALX 3,404a et SECARE 11,363b: apr. segar v.tr. «faucher (l'herbe)» Toulouse 1200, Brunel; Béz. 1300; HAlpes 1447, MeyerDoc; 1484, Fazy.

1 dalhar (prat)

[ca. 1238; cop. ca. 1390] Bigorre: A Sempey Marque a . . II pratz que deuen dalhar.

2 segar (erba) ab faus, segar (herba)

1203 [cop. 1542] SGaudens: Los homes de Sent-Gaudens an spleita de paisser [los bestiars e d-erba segar ab faus . . que-n pot home] fer en la honor de Landorta. 1289 [cop. $14^{\mathrm{e}} \mathrm{s}$.] Monségur: E si nulhs, o hom o femna, es trobadz de dies en l'autruy prat, per segar herba, que sia punitz a VI. sols de gatge.

\section{6 droit de faucher:}

1. «droit de couper le bois et l'herbe»

talh et dalh synt.m. [ca. 1275; cop. ca. 1460] ForGén, ForsB[OG] 292[264]; [1288; cop. ca. 1460] ForGén, ForsB[OG] 292[263] = LR; Lv [sans cont.].

À rattacher à FEW *DACULU, -A 3,2b; FEW TALIARE $13^{1}, 51 \mathrm{~b}$ : apr. talh et dalh «droit de coupe dans les bois»; Lv 8,24b sub talh 5 «Holzfällrecht»: abéarn. id. 1301 DocSPé; Rn 3,2a: [ca. 1400] ForsB; LR sub arrous 1: talh, dalh «droit de coupe, de fauchage» [s.d.] ArchBPyr.

$\diamond$ Rem. Le texte de ForsB explique: le bois qu'on a droit de couper (talh) doit servir aux maisons et aux autres bâtiments couverts, granges, pressoirs, ou à faire la clôture des châteaux, et les herbes qu'on a droit de faucher (dalh) doivent servir à la nourriture du bétail.

1 talh et dalh

[1288; cop. ca. 1460] Béarn: quant dus locx an padoent l'un sober l'autre et talh et dalh.

\section{7 fourche:}

1. «corvée nécessitant l'emploi de la fourche»

forca $\mathrm{f}$. [ca. 1180; cop. ca. 1390] CensIbos, CartBigRC 34[19,10;16;17;21].

FEW FURCA 3,884b: apr. forca «instrument à long manche de bois ou de fer, pour retourner le foin, la paille, remuer le fumier, charger les gerbes, etc.», cf. ib.: mfr. forche «corvée d'un homme qui doit venir avec sa fourche pour faner le foin»; Lv 3,538b: cf. agasc. forqua ferria $\mathrm{f}$. «Heu-, Mistgabel» ca. 1450 TarifBord; LR sub hourque: abéarn. forca «fourche» [source non indiquée]. 
2. «travail à la fourche (ou fourchée ?)»

forcada f. [ca. 1180; cop. ca. 1250] CensIbos, CartBigRC [19,10: var. B de forca ms. Pa, ci-dessus 1].

$\varnothing$ FEW FURCA 3,885b: béarn. hourcade «ce qu'on prend en une fois avec la fourche» $[=\mathrm{LR}]$.

1 forca

[ca. 1180; cop. ca. 1390] Ibos: forca (v. sub faux 2731,1).

2 forcada

[ca. 1180; cop. ca. 1250] Ibos: A Clauarie .II. mois de froment, .XVIII. de per porc e per molto e forcada e falz e flaged e cauad albergas, pa far e legna.

\section{8 râteau:}

1. arrested $\mathrm{m} .:$ arrested de fer 1268 Gabarret, RecGascL $87=\mathrm{Lv}$, rastet [av. 1300; cop. 1489] CoutBazasB, ArchGir 15,113[75].

FEW RASTELLUS 10,94a: agasc. restet $\mathrm{m}$. «outil d'agriculture et de jardinage, consistant en une traverse munie de dents et qui sert à ramasser le foin, les feuilles tombées, etc.»; Lv 7,40b sub rastel 1 «Rechen, Harke»: agasc. restet 1493 InvGrim; LR sub arrastèt: abéarn. rasteg «râteau» [s.d.] ArchBPyr.

\section{1 rastet}

[av. 1300; cop. 1489] Bazas: si no rastera lo blat, si rastet hy falh, que s'en deu far?

\section{9 râteler, ratisser:}

1. rasterar v.tr. [av. 1300; cop. 1489] CoutBazasB, ArchGir 15,113[75].

FEW RASTELLUS 10,96b/97a: apr. rastelar v.a «ramasser avec le râteau», Gers, béarn. arresterá; Lv 7,41b: agasc. rasterar «harken» 1396 EtMarm.

1 rasterar

[av. 1300; cop. 1489] Bazas: rastera (v. sub râteau 2738,1).

\section{0 foin:}

1. «herbe séchée pour la nourriture du bétail»

fen m. 1238 [vid. 1278; cop. 14 s.] PrivEDM[D], ArchGir 3,121; [ca. 1238; cop. ca. 1390] CartBig v. far fen sub faner, couper et récolter les foins 2743,1; [1255; cop. $16^{\mathrm{e}} \mathrm{s}$.] CoutLaRéoleG, ArchGir 2,252[2]; [1258; cop. 16 ${ }^{\mathrm{e}} \mathrm{s}$.] CoutLaRéoleG, ArchGir 2,269[60]; [1265; cop.] CoutSarrant, CoutGersB 41; 1270 Lectoure, Doc- 
GalardN 41,32;35; 1270 [cop. 1287] CoutSauve, CoutGersB 188[23]; [ca. 1280; cop. 1343] EtLect, ArchLectD 59[9];65[40]; 1283 ChartAlbrM 125; 1283 [cop. $14^{\mathrm{e}}$ s.] StatMonségurM, ArchGir 5,35;38 (v. aussi feys de fen sub botte de foin 2745,1); 1296 [n.st.] ChartAlbr v. feychs de fen sub 2745,1; [13 s.; cop.] CoutMeilhanB, ArchGir 25,144[36]; ca. 1300 StatCaudecosteB, ArchGir 31,226[25], fee 1295 [cop. ca. 1330] CoutFezB, ArchGir 27,388[30].

FEW FENUM 3,455a: béarn. hee «foin» [= LR: 1583 Salette]; LR: abéarn. fen [av. 1400; cop. 15 s.] ForAspe, ForsB[OG] 542[18: fee (cop. ca. 1460)]; 1520 CoutSoule, feis pl. 1520 CoutSoule, ib. sub prat: fen sg. [s.d.] ForsNav; sub palhe;fayot: fee [s.d.] ArchBPyr; ca. 1377 RôlesGPhébus.

1 fen

1238 [vid. 1278; cop. $14^{\mathrm{e}}$ s.] Entre-deux-Mers: es enjungit a las maysons religiosas que amenian senglas baquas a la cort del senescaut et lo fen et la palha de la terra son prees (v. aussi sub paille 2786,1).

\section{1 prairie à foin:}

1. «pré à foin, terre où l'on recueille du foin»"

feaa m. 1278 [cop. ca. 1460] ForOlDC 103a[2: trad. «fenaison»]; ForsB[OG] 496 [2: «pré, prairie à foin»] $=\mathrm{Lv}$ «Heuwiese»; LR «terre où l'on recueille le foin», fear 1278 [cop. $15^{\mathrm{e}}$ s.] ForOl, ForsB[OG] 498[2: var.] = LR; 1281 [cop. 14 s.] DocSPéB 396.

FEW FENUM 3,455a: aland. fenhar «pré où l'on recueille du foin», béarn. heaa «pré», Teste hena; Lv 3,435a sub fenar: abéarn. feaa «Heuwiese» 1424 ArtBéarn; 1301 [cop. 14 s.] DocSPé; LR sub mamudà: id. «pré» [s.d.] ArchBPyr., ib. sub broche: fear [s.d.] ArchBPyr; cf. ib. sub heaa: Heaas 1343, Feaas 1385 (tous deux nom d'une commune de l'arrond. d'Oloron).

1 fear

1281 [cop. 14 s.] Pau: Empero si lo vestiar dels homis d'Asson e d'Iguon fasen ... tale de fears ne de fenestres, que ac deven adobar.

\section{2 fenaison:}

1. fenar m. 1278 [cop. 1551] CartOlDC 102a[2;3]; ForOl, ForsB[OG] 498[2] = Lv $3,434 \mathrm{~b}$ «heuen» $=\mathrm{LR}$ «fenaison».

ø FEW FENUM 3,459.

1 fenar

1278 [cop. 1551] Oloron: Examentz guadanha deus prohomis d'aqueste terre que ayan herba et pastenc ad obs de lors jumentz ses dampnage de mes et de fenar. 


\section{3 faner, couper et récolter les foins:}

1. far fen loc.verb. [ca. 1238; cop. ca. 1390] CartBigRC 105[71,89].

À rattacher à FEW FENUM 3,455a.

1 farfen

[ca. 1238; cop. ca. 1390] Bigorre: A Ast los casals qui i sson deuen fen far e ga[ria] e agnet e ciuada e palha.

\section{4 meule de foin, tas de foin:}

1. feer m. 1143 [n.st.; cop. ca. 1310] CoutCornS [5: trad. «meule de foin»], fener 1295 [cop. ca. 1330] CoutFezB, ArchGir 27,388[30: trad. «fenière» mais plutôt «tas, meule de foin»].

FEW FENUM 3,457a: apr. fenier «fenil» Lv; Pans; etc., Lagraulet heè «tas de foin»; Lv 3,444b: agasc. feney «Heuhaufen; Heuschauer, -boden» $14^{\mathrm{e}} \mathrm{s}$. CoutGont.

1 feer

1143 [n.st.; cop. ca. 1310] Corneillan: Lo casau e-u palher e-1 feer deven ester seg[urs ?] e dedens justizie.

\section{5 botte de foin:}

1. feys de fen synt.f. 1283 [cop. $14^{\mathrm{e}}$ s.] StatMonségurM, ArchGir 5,37, feychs de fen 1296 [n.st.] ChartAlbrM 79[51].

À rattacher à FEW FENUM 3,455a et FASCIS 3,428a: aland. fuex «botte, assemblage de plusieurs choses de même nature liées ensemble», abéarn. foeix, feys [= LR sub hèix: foeixs de fen «botte de foin» [av. 1400] ForsB[OG] 542[18]: lit feix de fee (var.fen)] et ib.,428b: apr. fais «botte (de foin)» HAlp., MeyerDoc; Lv.

1 feychs de fen 1296 [n.st.] Bordeaux: I feychs de fen que-us en deu dar e rendre cascun an, aissi cum li lurs autre afeuat de la deita parropia.

3. Les travaux du blé

\section{6 champ:}

1. camp m. [1158; cop. $14^{\mathrm{e}} \mathrm{s}$. (cont. lat.)] CartSordeR 117; [12 $\mathrm{s}$; ; cop. ca. 1390] CartBigRC 25[9,16;20]; 1238 [cop. 14e s.] PrivEDM[D], ArchGir 3,117; 1248 1734 
SGaudens, RecGascL 9[9]; 1278 [cop. ca. 1460] ForOlDC 125a[24]; 1278 [cop. 1551] CartOloron, ForOlDC 124a[24]; [ca. 1280; cop. 1343] EtLect, ArchLectD 72[79]; 1282 [cop. 1356] MartOrthB 96; 1296 [cop. 16 ${ }^{\mathrm{e}}$ s.] Bayonne, EtR 2,81[3]; [13 ${ }^{\mathrm{e}}$ s.; cop.] CoutMeilhanB, ArchGir 25,144[36], cam 1282 [cop. 1356] MartOrthB 95; 1298 [cop. 1336] EtBay 62[34].

FEW CAMPUS 2,156a: apr. can «pièce de terre destinée à être cultivée, non enclose de murs»; LR: abéarn. camp «champ» $15^{\mathrm{e}} \mathrm{s}$. BaronBéarn, ib. sub bruxoc;campèstre: [s.d.] ArchBPyr; [ca. 1490] LRougeOssau.

2. agre m. 1260 Bagnères-de-Bigorre, LuchaireEt 295.

FEW AGER 24,257b: abéarn. agre «champ» 1260, RohlfsAger 10; DOM 320 b sub agre $_{2}$.

3. terradge $\mathrm{m}$. 1260 Bagnères-de-Bigorre, RecGascL 31 [gloss «redevance féodale» à corr.] $=$ Lv 8,189a sub 1 «Acker, Feld» [avec ?].

$\varnothing$ TERRA $13^{1}, 251 \mathrm{~b}$ : cf. langr. terrage «domaine».

4. «terre cultivable, champ»

cauzas obrarias f.pl. 1262 [cop. $16^{\mathrm{e}}$ s.] Bordeaux, ArchGir 53,3.

À rattacher à FEW CAUSA 2,541 et OPERARI 7,365a.

1 camp

[1158; cop. $14^{\mathrm{e}}$ s.] Sorde [cont. lat.]: et dedit Sancto-Johanni viridarium a infer dus camps, ubi poterant esse CC pomaria.

2 agre

1260 Bagnères-de-Bigorre: locau dootiu ... nostre maj na Comdor l'aue dad e confermad a la auant dite mason ... so es asaber ... los terradges e-ls agres, ..., los pradz e-ls pastencs.

3 terradge

1260 Bagnères-de-Bigorre: terradges (v. ci-dessus sub 2, texte dans LuchaireEt: var. de RecGascL et Lv).

4 cauzas obrarias $\mathrm{pl}$.

1262 [cop. $16^{\mathrm{e}}$ s.] Bordeaux: si en Guitart de Borc o suy her . . . bolon o poden de la medissa palu que sere au medis en Guitart aissi cum es auandeyt a pradar, o far aubaredas, o cauzas obrarias, o vinhas.

\section{7 champ de fourrage:}

1. ferragnas f.pl. ca. 1180 [cop. ca. 1390] CensIbos, CartBigRC 34;35[19,25;44], gloss «terres réservées à des plantes fourragères ou à des cultures de choix» avec 
comment; RecGascL 13[11], gloss «forge» à corr. = Lv 3,468a sub ferranha [sans déf.: „Ich verstehe die Stelle nicht"].

ø FEW FARRAGO 3,421b; cf. farragnoun m., ferragnoun «petit clos de fourrage en vert» $\mathrm{M}$.

1 ferragnas $\mathrm{pl}$.

ca. 1180 [cop. ca. 1390] Ibos: Arramon de Bene. VI. d. per las ferragnas.

\section{8 convertir en champ:}

1. acampir v.tr. 1258 et 1259 [cop. $14^{\mathrm{e}} \mathrm{s}$.] LOr v. terre [a] acampir sub terre inculte, en friche 2709,$5 ; 1258$ et 1259 [cop. $14^{\mathrm{e}} \mathrm{s}$.] LOr v. terre acampide sub terre cultivée 2710,4 .

FEW CAMPUS 2,157b: abéarn. acampir «convertir une terre en champ»; LR: LOr [v. ci-dessus] et [s.d.] ArchBescat; DOM 1,73b [ArchBPyr à corr. ArchBescat] = LR; RecGascLGloss: campir «mettre en culture» 1258 [cop. $14^{\mathrm{e}} \mathrm{s}$.] LOr [à corr. acampir].

\section{9 préparer la terre:}

1. aparelar v.tr. $12^{\mathrm{e}} \mathrm{s}$. [cop. $14^{\mathrm{e}} \mathrm{s}$.; cont. lat.] CartSordeR $138 ; 139[162]=\mathrm{LR}$ sub aparelha.

FEW *APPARICULARE 25,25b: cf. lang. gasc. aparelhá «. . .; préparer; . . .» $\mathrm{M}$, land. aprelhá «préparer».

1 aparelar

$12^{\mathrm{e}} \mathrm{s}$. [cop. $14^{\mathrm{e}} \mathrm{s}$.] Sorde [cont. lat.]: Lo casau ke ten Bergon Arnaut dat VI panes, . . debet arar, aparelar, carreiar, femeiar, sarclar, segar (v. aussi sub travailler, labourer (la terre, etc.) 2750,5).

2750 travailler, labourer (la terre, le champ, la vigne) [v. aussi cultiver 2680]:

1. laurar v.tr. [ca. 1160] Comminges, Brunel 94[97]; 1278 ForOlDC 100b avec comment., note IIa; 1282 [cop. 1356] MartOrth v. terre laurade sub terre labourée 2753, 4; 1295 [cop. ca. 1330] CoutFez v. bestia terra laurant sub bête de labour 2755,1; laborar 1259 [cop. ca. 1320] CartBlanc, CartAuchL 2,224[8]; 1270 Lectoure v. terra laborada sub terre labourée 2753,4; 1276 [n.st.] Bordeaux, ArchGir 43,169 [172]; 1278 [cop. $15^{\mathrm{e}}$ s.] CartOssauT 57[= LR sub countrast];59; [av. 1300; cop. 1489] CoutBazasB, ArchGir 15,111[71];112[72;73;74;75].

FEW LABORARE 5,103b: apr. laorar, laurar, lavorar «labourer» Rn; Lv; 1736 
etc. et ib. 105a: béarn. labourá «labourer»; LR sub laura;boarie: abéarn. laborar «id.» $15^{\mathrm{e}} \mathrm{s}$. BaronBéarn; 1551 ForsB, ib. sub esfruta: [s.d.] ArchBPyr; sub acampir: laurar [s.d.] ArchBescat.

2. obrar v.tr. 1257 [n.st.] CartSCrD, ArchGir 27,254[274]; 1279 Bordeaux, ArchGir 43,205; 1279 [n.st.] Bordeaux, ArchGir 43,201; 1283 [n.st.] CartSCrD, ArchGir 27, 222[239]; [av. 1300; cop. 1489] CoutBazasB, ArchGir 15,150[186]; ca. 1300 StatCaudecosteB, ArchGir 31,230[46: trad. «bêcher (la vigne)»].

FEW OPERARI 7,365a: apr. obrar «cultiver (la terre)» avec n3 [= Lv 5, 454b sub 5 «(Land) bearbeiten, bestellen» déb. 13 ${ }^{\mathrm{e}}$ s. HomélProv]; cf. LR sub oubra: béarn. (casau) bien oübrat «(jardin) bien travaillé»; v. aussi l'exemple de ca. 1390 dans LR, ici sub façon donnée au sol, à la terre 2751,1, var.

3. serbir v.tr. 1257 [n.st.] CartSCrD, ArchGir 27,254[274]; [ca. 1280; cop. 1343] EtLect, ArchLectD 69[68] = Lv 7,621b/622a sub servir 5 «(ein Feld) bestellen, bebauen» [avec ?].

FEW SERVIRE 11,537a: apr. servir v.a. «labourer, cultiver (un champ)» $13^{\mathrm{e}} \mathrm{s}$.

4. «labourer les vignes»

a) coutinar (binhas) v.tr. 1283 [n.st.] CartSCrD, ArchGir 27,222[239]; coytinar (heretatz) [1273] ACoutBay, Bal 2,656 [formes aberrantes ?].

ø FEW *COHORTILE 2,854b ?; p.-ê. croisement avec les verbes du type apr. coltivar, coitivar (< COLERE FEW 2,886b; Rn 2,443b; Lv 1,289b) ou mauvaise lecture pour coutiuar, coytiuar, v. les formes sub cultiver 2680 , 2 ? Cf. aussi cependant abéarn. coytinar (laquoau binhe los diitz Berdolet e sa molher prometon obrar e coytinar de totes obres que a binhe s-aparthien) 1389 Cézeracq, NotPrincBéarnB, Annexes VII[43, fo 41v,7].

b) acortinar (vinhas) v.tr. [forme aberrante ?] 1279 [n.st.] Bordeaux, ArchGir 43,201; 1279 Bordeaux, ArchGir 43,205.

$ø$ FEW *COHORTILE 2,854b: cf. pic. courtiner «jardiner»; mfr. acourtillier «cultiver un jardin» $14^{\mathrm{e}} \mathrm{s}$. Ou plutôt mauvaise lecture pour acortivar ou même acoitivar, ici sub cultiver 2680,3 ?

c) far (la binha) v.tr. [av. 1300; cop. 1489] CoutBazasB, ArchGir 15,112[73].

$ø$ FEW FACERE 3,346b.

5. «travailler la terre avec une charrue»

arar v.tr. $12^{\mathrm{e}} \mathrm{s}$. [cop. $14^{\mathrm{e}} \mathrm{s}$; cont. lat.] CartSordeR 138;139[162] = LR sub arar; aparelha;148[175]; arar amb ayre loc.verb. [1288; cop. ca. 1300] CoutMontsaunèsM $18 \mathrm{a}$. 
FEW ARARE 25,81b: apr. abéarn. arar v.a. «labourer», pour ayre v. ici sub charrue 2756,2 .

6. «travailler la terre avec des bœufs»

boiar v.tr. [12 $2^{\mathrm{e}}$ s.; cop. $14^{\mathrm{e}} \mathrm{s}$.] CartSordeR $148[175]=\mathrm{LR}$.

$ø$ FEW BOS 1,445b; LR sub bouya: abéarn. boyar «travailler avec des bœufs, labourer» [s.d.] ArchBPyr.

1 laborar, laurar

1259 [cop. ca. 1320] Auch: e-1 dit P. deu Lavai dab sos parenz dizen que prenen la demne de lors arades de zo que laboraven e-l territori de Lobencs (v. aussi sub donner une deuxième façon aux vignes 2823,1). - 1278 Oloron: En comensament (de e autreia a lor e a totz los qui au diit laug) de Ferrere bieren poblar places e terres cada VI iornades ob de laurar.

\section{2 obrar}

[av. 1300; cop. 1489] Bazas: Item, si lous [= bœus ou baccas] trobas en binha o en blat obradas, auras la malfeyta que sera presada per homes (v. aussi ci-dessous sub $3 ; 4 a ; b)$.

\section{3 serbir}

1257 [n.st.] Bordeaux: que de-ux medis aubars et bimes poden far à lur boluntat, serbida et obrada la medissa binha.

4a coutinar

1283 [n.st.] Bordeaux: . . . an promes las medissas binhas obrar et coutinar be et leiaument de marra et de coutet.

$4 \mathrm{~b}$ acortinar

1279 [n.st.] Bordeaux: laquau vinha . . . deven obrar e acortinar ben e leaument de destrau e de conge.

4c far (la binha)

[av. 1300; cop. 1489] Bazas: si lodict home no fey la binha, que pot far lo senhor?

5 arar

$12^{\mathrm{e}} \mathrm{s}$. [cop. $14^{\mathrm{e}} \mathrm{s}$.] Sorde [cont. lat.]: Lo casau Gassie Furt dat VI panes, ... debet arar, aparelar, carreiar, femeiar, sarclar, segar (v. aussi ci-dessous 6; sub préparer la terre 2749,1$)$.

6 boiar

[12 s.; cop. $14^{\mathrm{e}} \mathrm{s}$.] Sorde [cont. lat.]: Curtei, casau d'Urdassen, ... debet etiam boiar, arar.

\section{1 façon donnée au sol, à la terre:}

1. obras f.pl. [av. 1300; cop. 1489] CoutBazasB, ArchGir 15,112[73];113[75]. 
FEW OPERA 7,358b: cf. mfr. frm. auvres f.pl. «labours, façons de vigne, etc.» Pin 1562; dp. Laur 1704; cf. LR sub a (prép.): abéarn. obras «travaux (pour améliorer la terre ou pour planter)» ca. 1390 JugesMorl, ForsB[OG] 480[294: obres (ad adobar o a plantar); avec var. (cop. ca. 1460): obres (que-y fasse obrar o de plantar)].

1 obras pl.

[av. 1300; cop. 1489] Bazas: si lo senhor se bol que seguira lo cours de las obras de las binhas.

\section{2 donner une quatrième façon à la terre:}

1. quartar v.tr. 1265 [cop. $17^{\mathrm{e}} \mathrm{s}$.] CoutLarrC 121[15].

$\varnothing$ FEW QUARTUS 2,1426b; Rn 5,6b: cartar (la terra) «quarter, donner une quatrième façon, en parlant du nombre des labours donnés à la terre» 1315 Doat [alang. ou agasc.?].

1 quartar

1265 [cop. $17^{\mathrm{e}} \mathrm{s}$.] Larrazet: si-l lauraire y metia mais buous per quartar ni per cubrir no deu dar mas per un parelh.

2753 terre labourée [v. aussi terre cultivée 2710]:

1. arade f. 1259 [cop. ca. 1320] CartBlanc, CartAuchL 224;225[8].

FEW ARARE 25,82a: apr. arada f. «. . .; terre labourée» Rn; Pans 5, lang. gasc. arado «champ labouré; ... .».

2. laborage m. [av. 1300; cop. 1489] CoutBazasB, ArchGir 15,151[190] = Lv 4, 295 b sub 2 «Ackerfeld».

FEW LABORARE 5,105b: apr. laboratge «champ labouré ou à labourer».

3. laurat m. 1278 [cop. 1460] ForOlDC 101a[1].

FEW LABORARE 5,104a: apr. laurat m. «champ labouré» [= Lv 4,337b sub 1 «bestelltes Feld»: alang. 14 ${ }^{\mathrm{e}} \mathrm{s}$. Narbonne]; LR: béarn. los lauratz pl. «les terres cultivées».

4. terra laborada synt.f. 1270 Lectoure, DocGalardN $4^{1}, 32$; terre laurade 1282 [cop. 1356] MartOrthB 95.

À rattacher à FEW TERRA $13^{1}$,245a et LABORARE 5,104b/105a; 103b/104a.

1 arade

1259 [cop. ca. 1320] Auch: e dis per dit que-u dit P. nuls temps d'aici evant, ne-us 
ditz sos parenz, no prengen demne de los arades deu teritori de Lobencs (v. aussi sub travailler, labourer (la terre, etc.) 2750,1).

3 laurat

1278 [cop. 1460] Oloron: (et de-us padoence a totz e-us hermis et e-us coutz) et e-us lauratz aixi cum aus homis de la medixe biele.

4 terra laborada

1270 Lectoure: o en vignas, o en cazals, o en terras cotas, o no cotas, hermas, o laboradas.

\section{4 labour:}

1. «travail de labourage»

labor f. 1278 [cop. $15^{\mathrm{e}}$ s.] CartOssauT 57; 1296 [cop. 1406] LVertBénacB 207[20: trad. «labourage»].

FEW LABORARE 5,105a: béarn. labou m. «labour» [=LR: aussi abéarn. labor «labourage» $14^{\mathrm{e}} \mathrm{s}$. [cop. $15^{\mathrm{e}} \mathrm{s}$.] ForGén, ForsB[OG] 278[242: var.]; «labour» [s.d.] ArchMontaut]; Lv 4,294a sub 3 «Feldbestellung, Pflügen»: alang. [1333; cop. 1667] Rabastens, MonogrTarnR 4,257, ib. 337a sub lauransa 1: [s.d.] RechAlbi.

1 labor

1296 [cop. 1406] Tarbes: Item, autreian que-1 deuen tier bende de ciuade al preds que a Tarbe se bene, tot home e tote femne qui de sa labor la aie.

\section{5 bête de labour:}

1. bestia terra laurant synt.f. 1295 [cop. ca. 1330] CoutFezB, ArchGir 27,387[27]. À rattacher à FEW BESTIA 1,340b, TERRA $13^{1}, 245 \mathrm{a}$ et LABORARE 5,103b. 1 bestia terra laurant 1295 [cop. ca. 1330] Fezensaguet: Item fot ordenat e autreyat, . . per lodit mossenhor lo bescomte, que degun no sia penherat per rason d'alguna obligation, ... de las bestias terra laurant.

\section{6 charrue:}

1. araire $\mathrm{m} .1265$ [cop. $17^{\mathrm{e}} \mathrm{s}$.] CoutLarr v. ferramens que a l'araire s'aperteno sub ferrures, pièces de la charrue 2757,1, arayre [1288; cop. ca. 1300] CoutMontsaunès v. esturmens pertenens as arayre sub 2757,2, arair 1289 [cop. 14 s.] Monségur v. estrumenta de son arair sub 2757,3. 
FEW ARATRUM 25,83a: apr. araire m. «charrue simple, sans avant-train et sans roues», Agen aray f. "charrue», gasc. arai 1587, Teste arai avec n1; cf. Palay: Gers aray m.; M: gasc. id.

2. «charrue attelée (de bœufs, d'ânesses ou d'ânes)»

araire (de buous) m. [1288; cop. ca. 1300] CoutMontsaunèsM 18a[14], ayre (de saumas o d-azes) [1288; cop. ca. 1300] CoutMontsaunès v. arar amb ayre sub travailler, labourer (la terre, etc.) 2750,5.

$ø$ FEW ARATRUM 25,83a: cf. Agen aray f. «charrue», gasc. arai 1587, Teste id.

$\diamond$ Rem.: ayre: dans la version de 1500 figure aray [ab ung aray d-ases ou de saumes] (CoutMontsaunèsM 18b).

2 araire, ayre

[1288; cop. ca. 1300] Montsaunès: totz hom que are ... am .I. araire de buous, done, cascu an . . . . I. sestier de mestura ... et ayso meteys done qui arara amb ayre de saumas o d azes.

\section{7 ferrures, pièces de la charrue:}

1. ferramens que a l'araire s'aperteno synt.m.pl. 1265 [cop. $17^{\mathrm{e}} \mathrm{s}$.] CoutLarrC $121[15]$.

À rattacher à FEW FERRAMENTUM 3,468a, ARATRUM 25,83a et APPERTINERE 25,34a.

2. esturmens pertenens as arayre synt.m.pl. [1288; cop. ca. 1300] CoutMontsaunèsM 18a.

À rattacher à FEW INSTRUMENTUM 4,726b, PERTINERE 8,283b et ARATRUM 25,83a.

3. estrumenta de son arair synt.f. 1289 [cop. $14^{\mathrm{e}} \mathrm{s}$.] Monségur: CartMonségurM ArchGir 5,30.

À rattacher à FEW INSTRUMENTUM 4,726b et ARATRUM 25,83a.

1 ferramens que a l'araire s'aperteno pl.

1265 [cop. $17^{\mathrm{e}} \mathrm{s}$.] Larrazet: ferramens tots aquels que a l'araire s'aperteno (v. sub aiguillon (du bouvier) 2727,1).

2 esturmens pertenens as arayre $\mathrm{pl}$.

[1288; cop. ca. 1300] Montsaunès: faure bon e competent que sapcha far e fargar esturmens pertenens as arayre.

3 estrumenta de son arair

1289 [cop. $14^{\mathrm{e}} \mathrm{s}$.] Monségur: si nulhs hom o femna passa a travers les autruis 
vinhes o cazals, que sia punitz ..., si donc no anava a font, o culhir estrumenta de son arair, e que ac poscos mostrar a les gardes.

\section{8 coutre:}

1. coutre $\mathrm{m} .1265$ [cop. $17^{\mathrm{e}} \mathrm{s}$.] CoutLarrC $121[15]=\mathrm{Lv} 7,202 \mathrm{a}$ sub relha 1 , coute [1288; cop. ca. 1300] CoutMontsaunèsM 53.

FEW CULTER 2,1502b: apr. coltre «fort couteau fixé en avant du soc de la charrue, pour fendre la terre», Gers coute «coutre», béarn. coudre [= LR: aussi abéarn. codre «coutre» [s.d.] ArchBPyr, ib. sub arrasère: id. ArchBPyr, ib. sub ustilhes: coudre 1354 ArchBPyr].

2. gazeu m. [texte: gazen] 1265 [cop. $17^{\mathrm{e}} \mathrm{s}$.] CoutLarrC 121[15] = Lv 4,98a sub gazel [sans déf.] corr. gazen en gazeu; ib. 7,202a sub relha 1 .

FEW 22²,42a sub coutre: Toulouse gazel m. «coutre»; cf. M sub gazèu, gazèl «coutre de charrue», en Lauragais.

$\diamond$ Rem. Sens douteux; v. aussi FEW 22²,42a sub soc: Agen gaben m. «soc de la charrue»; cf. M: gaben «soc de charrue», en Agenais et Rouergue et Lv 4,91a: aoccit. gavenc, guavenc «Pflugschar».

1 coute

[1288; cop. ca. 1300] Montsaunès: coutes pl. (v. sub aiguiser 2732,1).

\section{9 soc de charrue:}

1. relha f. 1265 [cop. $17^{\mathrm{e}}$ s.] CoutLarrC 121[15] = Lv 7,202a sub 1 «Pflugschar»; 1270 [cop. 1455] CoutMontoussD 650[43]; [1288; cop. ca. 1300] CoutMontsaunèsM 53.

FEW REGULA 10,218a: apr. relha f. «soc de charrue» Provence, Albi, lim. gasc., Lv; etc., abéarn. arelha, Gers arreilho, Lagraulet rélho, Arrens arrélhas pl. «soc (formé de 2 parties)» Par 19, Aspe, land. arrélhe «soc», Vic-Bilh relhe [= LR, ib. sub arelhe: abéarn. arelhe «petite charrue» [s.d.] ArchBPyr].

$\diamond$ Rem. V. aussi sub coutre 2758,2.

1 relha

1270 [cop. 1455] Montoussin: et ab asso sobredit le faure deu agudar leyalment a bona fe sobre sagrament et [las forgas] deben aguda las relhas navas et far les agulhos que obs auran (v. aussi sub aiguiser 2732,1). 


\section{0 sillon:}

1. «ados entre deux sillons»

arregatge m. 1274 [cont. lat.] AnglGuyB 283[628], gloss «arregia, en gascon arregatge, rège ou sillon planté en vignes ou semé en blé».

ø FEW gaul. *RICA 10,389a: dérivé de Teste arrègue «sillon», etc. [formes phonétiques] (ib. 387b) du type de afr. mfr. riage m. "pièce de terre renfermée entre 2 sillons profonds" $13-17^{\mathrm{e}} \mathrm{s}$. ?

1 arregatge

1274 Guyenne [cont. lat.]: tenet idem Arnaldus . . . in dicta parochia, arregatges in loco vulgariter appellato A Gita Pena, et prope ecclesiam unam peciam terre.

\section{1 semer, ensemencer:}

1. semiar v.tr. 1278 [cop. $15^{\mathrm{e}}$ s.] CartOssauT $59=$ LR sub semiá, semenar 1279 Bordeaux, ArchGir 43,205; (terra) semenada p.p.f. [av. 1300; cop. 1489] CoutBazasB, ArchGir 15,135[137]; [1300] CoutAure, Monl 6,58.

FEW SEMINARE 11,433b/434a: agasc. somiar v.tr. «semer» Dax 1480 [= Lv 7,554a sub 1 «säen»], béarn. semiá, semená, soumiá «semer; ensemencer», Bayonne id. Lagr, Dax id. 1734, land. id. «id.»; LR sub semiá: cf. abéarn. assemiar (la terre) «ensemencer» [s.d.] ArchBPyr, ib. sub laura;boarie: semiar «semer» $15^{\mathrm{e}} \mathrm{s}$. BaronBéarn.

2. aber semensa synt.verb. [av. 1300; cop. 1489] CoutBazasB, ArchGir 15,112[75] $=\mathrm{Lv} 8,590 \mathrm{~b}$ sub varech.

À rattacher à FEW SEMENTIA 11,430a.

3. cubrir v.tr. 1265 [cop. $17^{\mathrm{e}} \mathrm{s}$.] CoutLarrC 121[15].

FEW COOPERIRE 2,1146b: apr. cubrir «ensemencer et recouvrir la semence par le $3^{\text {e }}$ labour» Fazy 1,57, curbi Livres de raison, Bagnères curbi «recouvrir le blé semé» MSLP 13, 83.

4. far (fèves, seigle, etc.) v.tr. [av. 1300; cop. 1489] CoutBazasB, ArchGir 15,112 [75: trad. «semer»].

\section{ø FEW FACERE 3,346b/347a.}

\section{1 semiar, semenar}

1278 [cop. $15^{\mathrm{e}}$ s.] Morlaàs: que homis de Pau los blatz qui an semiatz . . . ne puscan lhevar seguramentz, et las terres qui an laborades pusquen semiar et coelher aquest an. - 1279 Bordeaux: e las avandeitas terras ben e leiaument semenar deus blatz que s'i combindran cascun an (v. aussi sub semence 2762,1). 
3 cubrir

1265 [cop. $17^{\mathrm{e}} \mathrm{s}$.] Larrazet: cubrir (v. sub donner une quatrième façon à la terre 2752,1).

4 far (faba, etc.)

[av. 1300; cop. 1489] Bazas: ung home que pren dus jornals de terra . . . et y pot far faba ou sese ou bessa.

2762 semence [add. à graine 382,3-3]:

1. semence f. [1300] CoutAure, Monl 6,58, semensa [av. 1300; cop. 1489] CoutBazas v. aber semensa sub semer, ensemencer 2761,2.

FEW SEMENTIA 11,430a: béarn. semence f. «semence», Bayonne soumence.

1 semence

[1300] Aure: aucun habitant ... non deu este pugnerat ... ny en blat ... ny semence despus ques fors de la maison per semenar.

\section{3 herser:}

1. arrasclar v.tr. 1296 [n.st.; cop. 1406] LVertBénacB 205[3] = Lv 7,38b sub rasclar 3 «eggen».

FEW *RASCLARE 10,82a: agasc. arrasclar «herser» 1296 [v. l'attestation ci-dessus], Lagraulet, Ferrère arrasclá, Arrens id. P 20; LR sub arrascla: abéarn. arrasclar «herser» [s.d.] ArchBPyr.

\section{4 laisser se reposer un an (de la terre):}

1. meuar bareyt bilh synt.verb. [av. 1300; cop. 1489] CoutBazasB, ArchGir 15, 112[75: trad. «mettre en guéret»] $=$ Lv 8,590b sub varech, bareyt [sans déf. avec comment.: ,Es handelt sich um das Ruhenlassen und Umbrechen des Ackers in einem von zwei Jahren '] qui, sans nécessité, propose menar [avec ?] pour meuar.

À rattacher à FEW VETULUS 14,360b, VERVACTUM 14,332b: béarn. $b a-$ rèyt «guéret», land. Gir. «jachère» ALF 1600 et MOVERE $6^{3}, 164 \mathrm{a}$ : apr. moure la terra, etc. «l'ouvrir, lui donner le premier labour» 1293; ca. 1350; 1512, MeyerDoc. 


\section{La moisson}

2765 champ de blé [add. à 783 blé sur pied, champ de blé]:

1. blat m. 1289 [cop. 14 e s.] Monségur, ArchGir 5,29; [av. 1300; cop. 1489] CoutBazasB, ArchGir 15,150[186];151[189]; ca. 1300 StatCaudecosteB, ArchGir 31, 221[4] [add. à 783,1-1].

FEW abfrq. *BLAD $15^{1}, 127$ b: béarn. blat 1692 [= LR sub blat et expressament «champ de blé, de seigle»] à corr. en: abéarn. 1520 CoutSoule.

2. bladar m. 1270 [cop. 1287] CoutSauve, CoutGersB 188[23] [add. à 783,1-3].

$\varnothing$ FEW abfrq. *BLAD $15^{1}$,129b: aland. bladar m. «blé sur pied» $1368[=\mathrm{Lv}$ 8,15 b sub tala: Mill].

3. bladi m. 1278 [cop. $15^{\mathrm{e}}$ s.] CartOssauT 57 [add. à 783,Ad 1-4].

$\varnothing$ FEW abfrq. *BLAD $15^{1}$,128a: cf. abéarn. bladade f. «blés sur pied» = LR [ici 783,1-4]: 1278 CartOssau [var. de bladi (contexte presque identique)].

\section{1 blat}

1289 [cop. $14^{\mathrm{e}} \mathrm{s}$.] Monségur: E si nulhs hom o femna es trobat en l'autruy casal o en vinha o en blat del fruit prenen, que sia punitz a VI. sols de gatge.

\section{2 bladar}

1270 [cop. 1287] LaSauvetat: Et si augus intrava de nueyt en vinha, o en ort, . . o o en bladar, e se prenia fruct, ... qu'en sia punitz alsdits senhors en cinq sos de morlaas.

\section{3 bladi}

1278 [cop. $15^{\mathrm{e}}$ s.] Morlaàs: Que los Ossales posquen peixer et jaser per Ponlong ... sentz tale far de bladi et de planters.

2766 moisson [v. aussi sub blé 782,5-2]:

1. «récolte des céréales, en particulier du blé»

meitz f. 1255 [cop. $16^{\mathrm{e}}$ s.] CoutLaRéoleG, ArchGir 2,252[2], mes 1278 [cop. 1551] [DAG 782,5-1: [1290] à corr.] CartOloron, ForOlDC 102a[3] = Lv 5,241a «Ernte»; LR.

FEW MESSIS 6²,51a: abéarn. mes f. «moisson».

2. culleita f. 1242 [cop. $15^{\mathrm{e}} \mathrm{s}$.] CartSSeurinB 199[220].

FEW COLLIGERE 2,899a: béarn. culhete «récolte» [= LR: Orthez 1670, PrivRègl].

3. culhidura f. 1270 [cop. 1455] CoutMontoussD 650[43]. 
ø FEW COLLIGERE 2,899a; Rn 2,434a: culhidura «cueillette» [= LvP avec ?] 1274, Doat [alang. ou agasc.?].

4. blatz m.pl. 1238 [vid. 1278; cop. 14e s.] PrivEDM[D], ArchGir 3,119; [1288; cop. ca. 1300] CoutMontsaunèsM 12a.

$ø$ FEW abfrq. *BLAD $15^{1}, 127 \mathrm{~b}$.

1 meitz

1255 [cop. $16^{\mathrm{e}} \mathrm{s}$.] LaRéole: Asso son les establimentz que foren juratz davant lo dit nostre senhor et davan la dita comunautat . . . de ardre hostals, o blat, o fens et de meitz et de trulhs.

\section{2 culleita}

1242 [cop. $15^{\mathrm{e}}$ s.] Bordeaux: E si aucuns hom acensava la meitat de la detma de Cairac ... aquet qui la deyma tindre s'en deu benir a Bordeu ... per pagar a la Sent Andreu, e aco deu far an per an avant la culleita.

\section{3 culhidura}

1270 [cop. 1455] Montoussin: aquellas forgas deben aber mesura comunal e-ls mesados que culhiran lo blat tres cestes per culhiduras.

\section{4 blatz pl.}

1238 [vid. 1278; cop. 14 ${ }^{\mathrm{e}}$ s.] Entre-deux-Mers: edz mandan l'ost quascun mees per pauc, sens aucuna causa, estan per sola occasion de extorssa la pecunia dels cavaleys et dels laboradors, e mayorament en lo temps dels blatz et de las vendemihas.

\section{7 garde des moissons:}

1. messaria f. [12 ${ }^{\mathrm{e}}$ s.; cop. ca. 1390] CartBigRC 30;31[14,4;5: ms. Pa: «gardiennage des récoltes sur pied, office de messier»], messarie [12 ${ }^{\mathrm{e}} \mathrm{s} . ;$ cop. ca. 1250$]$ CartBigRC [14,5: var. B].

$ø$ FEW MESSIS $6^{2}, 51 \mathrm{a}$.

2. messagaria f., mesegerya [1288; cop. ca. 1300] CoutMontsaunèsM 12a.

$ø$ FEW MESSIS $6^{2}, 51 \mathrm{a}$.

\section{1 messaria}

[12 ${ }^{\mathrm{e}}$ s.; cop. ca. 1390] Bigorre: Lo casal de Za Mola . . . deu pa far e messaria.

2 mesegerya, messagaria

[1288; cop. ca. 1300] Montsaunès: De la mesegerya. Item, vollem et ordenam que los cossols de la dicha vila de Monsaones . . . aian poder d'empauzar, e de costituir et d ordonar, la messagaria. 
2768 gardien des moissons, des récoltes:

1. messeguer m. 1255 [cop. 1341] CoutMontrOG 129[8]; messaguier [1288; cop. ca. 1300] CoutMontsaunèsM 12a;13a[«mességuier»].

FEW MESSIS 62,51a: agasc. messeguier «gardien de la moisson» $15^{\mathrm{e}} \mathrm{s}$., Mir; cf. aussi Palay sub messè: Vallée d'Aspe messeguè «messier, garde des cultures, des récoltes sur pied».

1 messaguier

[1288; cop. ca. 1300] Montsaunès: lo qual messaguier juret en la presencia del dit jugge e des cossols ... que el gardara fizelmen totas las meyshos et los blatz.

\section{9 moissonner:}

1. «faucher (scier) le blé»

segar v.tr. $12^{\mathrm{e}} \mathrm{s}$. [cop. $14^{\mathrm{e}} \mathrm{s}$.; cont. lat.] CartSordeR 112[184] = LR sub sarcla;138; 139[162]; RecGascL 73;74, assegar $12^{\mathrm{e}} \mathrm{s}$. [cop. 14 ${ }^{\mathrm{e}} \mathrm{s}$; ; cont. lat.] CartSordeR 138 [162]; RecGascL 73, secar [12 ${ }^{\mathrm{e}} \mathrm{s}$; cop. 14 ${ }^{\mathrm{e}} \mathrm{s}$. (cont. lat.)] CartSordeR 138[161].

FEW SECARE 11,363b sub I.1. «Mähen»: apr. segar v.a. «faucher (le blé)» Montpellier ca. 1140; Millau 1443; LR: béarn. sega «scier les blés, moissonner»».

2. «récolter des céréales, en particulier du blé»

culhir (lo blat) v.tr. 1270 [cop. 1455] CoutMontoussD 650[43], coelher (las terres) 1278 [cop. $15^{\mathrm{e}} \mathrm{s}$.] CartOssauT $59=$ LR sub coelhe.

FEW COLLIGERE 2,899a: béarn. coelhe «récolter»; LR sub culhi: abéarn. culhir «cueillir, récolter (des grains)» 1520 CoutSoule.

3. Ihebar (los blatz) v.tr. 1278 [cop. 15e s.] CartOssauT 59, lhevar 1278 [cop. $\left.15^{\mathrm{e}} \mathrm{s}.\right]$ CartOssauT 59.

FEW LEVARE 5,280b: apr. levar «récolter» rouerg. 1164, Brunel, béarn. lhebá «récolter le blé» [= LR: béarn. lhebar (los frutz) «récolter (les fruits)»].

4. amassar (blat) v.tr. 1250 CartSCrD, ArchGir 27,249.

FEW MASSA $6^{1}, 445$ b: béarn. amassá v.tr. «cueillir, ramasser (des fruits, des châtaignes)»; LR sub amassa: béarn. amassa lou fruut «faire la récolte du fruit».

1 secar

[12 $\mathrm{e}$ s.; cop. $14^{\mathrm{e}}$ s.] Sorde [cont. lat.]: Debet etiam sarclar, secar.

2 culhir (lo blat)

1270 [cop. 1455] Montoussin: e-ls mesados que culhiran lo blat [deben] tres cestes per culhiduras. 
3 lhebar (los blatz)

1278 [cop. $15^{\mathrm{e}} \mathrm{s}$.] Morlaàs: et qu'en agen lhebatz los blatz de qui au des XV de Marteror.

4 amassar (blat)

1250 Bordeaux: quant lo messatges de la gleisa Senta-Crois aura amassat blat o bin en l'auandeit desmari de Galhan e-us [ $=\mathrm{W}$. de La Casa e W. Centot] semondra que la angan, anar i deuen ... et portar lo blat e-u bin . . . en l'estatge aperada l'Estatge-de-la-Casa.

\section{0 moissonneur:}

1. segador m. [ca. 1180; cop. ca. 1390] CensIbos, CartBigRC 35[19,43] = Lv.

FEW SECARE 11,365b: apr. segador «celui qui coupe les blés» Montpellier, gasc., béarn. Arrens segadoú «moissonneur»; Lv 7,511a: abéarn. seguador «Mäher, Schnitter» ca. 1330 [cop. ca. 1410] HistSainte [= LR sub segadou «moissonneur»: aussi segador ib.].

2. mesado[r] m.: mesados pl. 1270 [cop. 1455] CoutMontoussD 650[43].

$ø$ FEW MESSIS $6^{2}, 51 \mathrm{a} / \mathrm{b}$.

3. estiuande $[\boldsymbol{r}]$ m.: estiuandes pl. ca. 1300 StatCaudecosteB, ArchGir 31,228[36: trad. «métivier»].

FEW AESTIVARE 24,234b: apr. estivandier m. «moissonneur» Bonis, Lv, Bigorre estiuandè.

2 mesado $[r]$

1270 [cop. 1455] Montoussin: mesados pl. (v. sub moissonner 2769,2).

3 estiuande $[r]$

ca. 1300 Caudecoste: tot glenador e tota glenayritz yches del estolha a la requesta del senhor o de la dona o dels estiuandes.

\section{1 premiers produits de la terre, première récolte:}

1. «(droit sur) les premiers produits de la terre, (redevance sur) la première récolte)»

primizie f. 1179 Montsaunès, RecGascL 5 [gloss «prémice»] = Lv 6,553b sub primicia, pre- «Erstlinge», premisia [1260; cop. ca. 1600] PrivGavarnie, TextBigM 90[4].

FEW PRIMITIAE 9,380b: abéarn. premicie f.pl. «premiers fruits de la terre ou du bétail»= LR: béarn. premisie «prémices, premiers produits de la terre, du bétail» 1628 PrivRègl. 


\section{1 premisia}

[1260; cop. ca. 1600] Gavarnie: per lasd. abbadies pren lad. mayso de gauernie de lasd. decimas, fora las premisias, car de aqueras ne pren stremat de tout lo blat quy y bien. 
